\title{
Limitierungsverfahren von Reihen mehrdimensionaler Kugelfunktionen und deren Saturationsverhalten
}

Von

H. Berens, P. L. Butzer und S. Pawelke*

\section{Inhaltsverzeichnis}

1. Funktionen auf dər Kugel

2. Konvergenz und Summation von Reihen von Kugelfunktionen 2.1. Allgemeines

2.2. Sätze vom Bernsteinschen und Jacksonschen Typ

3. Ein allgemeiner Saturatiossatz

4. Spezielle singuläre Integrale

4.1. Das singuläre Integral von Weierstraß

4.2. Mittelbildungen von Funktionen auf der Kugel

4. 3. Das singuläre Integral von Abel-Poisson

4.4. Das Summationsverfahren von Cesàro

5. Funktionenklassen auf der Kugel

5. 1. Das verallgemeinerte singuläre Integral von Weierstraß

5.2. Das verallgemeinerte singuläre Integral von Abel-Poisson

5. 3. Klassen von Funktioen auf $S^{k}$

5.4. Charakterisierungen für zonale Funktionen

Literaturverzeichnis

Die vorliegende Arbeit untersucht das approximationstheoretische Verhalten von Summationsprozessen von Reihen von Kugelfunktionen, sogenannten Laplace-Reihen. Zunächst wird die Theorie der besten Approximation auf der Kugel, also die Erweiterung der Sätze von D. Jackson und S. Bernstein, skizziert. Nimmt man nun spezielle Verfahren zur Summation von Laplace-Reihen, dann lassen sich auch hier Sätze vom Jacksonschen und Bernsteinschen Typ beweisen. Darüber hinaus zeigen viele Verfahren ein Saturations-

Received March 18, 1968.

Communicated by M. Urabe.

* Rheinisch-Westfälische Technische Hochschule Aachen. 
verhalten, d.h. es gibt eine nur vom Verfahren abhängige optimale Approximationsordnung. Das Saturationsproblem kesteht nun darin, diejenige Klasse von Funktionen (Saturationsklasse) zu bestimmen, welche genau von der optimalen Ordnung approximiert werden. Die Abelschen und Cesàroschen Summationsverfahren von Laplace-Reihen haben zum Beispiel solch ein Verhalten, beide Verfahren sind darstellbar als singuläre Faltungsintegrale auf der Kugel mit "zonalen" Kernen. So dienen allgemeine Saturationssätze für eine Klasse von singulären Integralen als Grundlage zu dieser Theorie. Die Charakterisierungen der Saturationsklassen erfolgen einerseits mit Hilfe von Mittelungen auf der Kugel, die dem Integralmittelwert in $E^{1}$, den sphärischen Mitteln in $E^{k}$ oder auch Lipschitzbedingungen entsprechen, andererseits lassen sich die Funktionen aus den Saturationsklassen als Integrale von verallgemeinerten Ableitungen (z.B. als Rieszpotentiale) darstellen. Detailliertere Charakterisierungen werden für "zonale" Funktionen auf der Kugel, d.h. für Funktionen, die invariant bei Drehungen um eine feste Achse sind, über "konjugierte" Funktionen im Sinne von Muckenhoupt und Stein gewonnen.

Die Theorie der Funktionen auf der Kugel ist in den letzten Jahren entscheidend weiterentwickelt worden. Die Theorie der Laplace-Reihen wird für die Kugel im dreidimensionalen Raum ausführlich von G. Sansone [62], in höheren Dimensionen von $C$. Müller [51] dargestellt. Die Summation von Laplace-Reihen untersuchten u.a. W. Rudin [61] und V.L. Shapiro $[64,65,66]$. Der zentrale Begriff der Faltung auf der Kugel stammt von A.P. Calderón und A. Zygmund [27], er wurde ausführlich von C. F. Dunkl [30] behandelt. Fraktionierte Integrale wurden von $R$. Askey und St. Wainger $[3, \mathrm{I}]$ untersucht, und der Begriff der konjugierten Funktion wurde von B. Muckenhoupt und E. M. Stein [49] von periodischen Funktionen einer Veränderlichen auf zonale Funktionen auf der Kugel erweitert. Dagegen mußte die Saturationstheorie für singuläre Integrale auf der Kugel ganz aufgebaut werden. Hierzu standen den Verfassern die wohl ausgebaute Theorie auf dem Einheitskreis 
in der Ebene, sowie die auf dem $k$-dimensionalen Euklidischen Raum bzw. auf dem $k$-dimensionalen Torus zur Verfügung. Hier sind die Arbeiten von P. L. Butzer $[14,15,18], H$. Buchwalter [11, 12], G. Sunouchi und C. Watari $[69,70], H$. Berens [5] und weiter die von R.J. Nessel [52, 53], R.J. Nessel und P.L.Butzer [23], R. J. Nessel und A. Pawelke [54], E. Görlich [35] u.a. zu nennen.

Die Arbeit entstand am Lehrsthl A für Mathematk an der Rhein.-Westf. Technischen Hochschule Aachen. Der Beitrag des letztgenannten Verfassers-ihm oblag auch die Ausarbeitung-wurde im Rahmen eines Forschungsvorhabens der DFG unterstützt.

\section{Funktionen auf der Kugel}

Mit $S^{k}$ bezeichnet man die Oberfläche der Einheitskugel im $k-$ dimensionalen euklidischen Raum $E^{k}, k \geq 3$, mit Mittelpunkt im Nullpunkt ; $u, x, y, \cdots$ sind Punkte auf $S^{k}$ und $(x, y)$ ist das Skalarprodukt von $x$ und $y$, betrachtet als Vektoren in $E^{k}$. Es werden nun einige Klassen von komplexwertigen Funktionen auf $S^{k}$ definiert. Mit $\boldsymbol{C}\left(S^{k}\right)$ bezeichnet man den Banachraum der auf $S^{k}$ definierten stetigen Funktionen mit der Norm

$$
\|f\|_{c} \equiv \sup _{x \in S k}|f(x)|,
$$

wobei die Stetigkeit bezüglich der euklidischen Metrik des Raumes $S^{k}$ definiert ist. $L^{p}\left(S^{k}\right), 1 \leq p<\infty$, ist der Banachraum der auf $S^{k}$ definierten und dort bezüglich des Oberflächenelements $d s$ zur $p$-ten Potenz integrierbaren Funktionen mit der Norm

$$
\|f\|_{p}=\left\{\frac{1}{\Omega_{k}} \int_{S^{k}}|f(x)|^{p} d s(x)\right\}^{1 / p}
$$

und $\Omega_{k}$, die Oberfläche der Einheitskugel im $E^{k}$, ist geben durch

$$
\Omega_{k}=\frac{2 \pi^{\lambda+1}}{(\lambda+1)}
$$$$
(2 \lambda=k-2) \text {. }
$$

Mit $\boldsymbol{L}^{\infty}\left(S^{k}\right)$ wird der Banachraum der auf $S^{k}$ definierten und dort wesentlich beschränkten Funktionen bezeichnet. Die übliche Norm ist 


$$
\|f\|_{\infty} \equiv \underset{x \in S^{k}}{\operatorname{wes} \sup }|f(x)| \text {. }
$$

Falls $f \in \boldsymbol{L}^{p}\left(S^{k}\right)$ ist, dann ist $f \in \boldsymbol{L}^{p^{\prime}}\left(S^{k}\right)$ mit $1 \leq p^{\prime}<p \leq \infty$.

Als Untermengen dieser Räume treten Funktionen auf $S^{k}$ auf, die invariant bei der Drehung um eine gegebene Achse durch einen fest gewählten Nordpol $p \in S^{k}$ sind. Man nennt sie zonale Funktionen. Es besteht ein Isomorphismus zwischen den zonalen Funktionen und den Funktionen $f(\theta)$ auf $0 \leq \theta \leq \pi$ ([30]). Zonale Funktionen sind auf den Mengen $\mathscr{Y}_{\theta}=\left\{x ; x \in S^{k},(p, x)=\cos \theta\right\}$ für ein festes $\theta \in[0, \pi]$ konstant. Ist der Nordpol $p$ durch $p=(1,0, \cdots, 0)$ und der Punkt $x^{\prime} \in S^{k}$ durch $x^{\prime}=(\cos \theta, \sin \theta, 0, \cdots, 0)$ gegeben, dann ist $h(x)=h\left(x^{\prime}\right)$ für alle $x \in \mathscr{Y}_{\theta}$, d.h. $h(x)=h_{1}(\cos \theta, \sin \theta)=f(\theta), 0 \leq \theta \leq \pi$.

Man erhält in diesem Fall die Räume der zonalen Funktionen $\boldsymbol{C}_{\lambda} \subset \boldsymbol{C}\left(S^{k}\right)$ mit der Norm $\|f\|_{\boldsymbol{C}_{\lambda}} \equiv \sup _{0 \leq \theta \leq \pi}|f(\theta)|, \boldsymbol{L}_{\lambda}{ }^{p} \subset \boldsymbol{L}^{p}\left(S^{k}\right), 1 \leq p<\infty$, $2 \lambda=k-2$, mit

$$
\begin{array}{r}
\|f\|_{p, \lambda} \equiv\left\{\frac{\Omega_{k-1}}{\Omega_{k}} \int_{0}^{\pi}|f(\theta)|^{p} d m_{\lambda}(\theta)\right\}^{1 / p}, \\
d m_{\lambda}(\theta)=(\sin \theta)^{2 \lambda} d \theta
\end{array}
$$

und $\boldsymbol{L}_{\lambda}^{\infty} \subset \boldsymbol{L}^{\infty}\left(S^{k}\right)$ mit $\|f\|_{\infty} \equiv$ wes $_{0 \leq \theta \leq \pi} \sup |f(\theta)| . \quad \boldsymbol{M}_{\lambda}$ bezeichnet die Menge der Borelmaße $\mu$ auf $[0, \pi]$, für die gilt

$$
\|\mu\|_{N_{\lambda}} \equiv \frac{\Omega_{k-1}}{\Omega_{k}} \int_{0}^{\pi}|d \mu(\theta)|<+\infty
$$

Mit $\boldsymbol{X}$ wird von jetzt ab immer einer der Räume $\boldsymbol{L}^{p}\left(S^{k}\right), 1 \leq p<\infty$, oder $\boldsymbol{C}\left(S^{k}\right)$ bezeichnet.

Harmonische Polynome sind wie folgt definiert. Sei $z$ ein Punkt in $E^{k}, Q_{n}(z)$ ein homogenes Polynom vom Grade $n$ in $E^{k}$, das harmonisch in $E^{k}$ ist, d.h. $\nabla^{2} Q_{n}(z)=0\left(\nabla^{2}\right.$ ist der Laplace-Operator in $\left.E^{k}\right)$, dann heißt $Q_{n}(z /|z|),|z|=(z, z)^{1 / 2}, x=z /|z|$, eine Kugelfunktion vom Grade $n$ und wird mit $Y_{n}(x)$ bezeichnet.

Man definiert den Laplace-Beltrami-Operator $\Delta$ auf der Kugel $S^{k}$ wie folgt. (s. z.B. [65, S. 213]o der [26, S. 34]). Ist $f(x)$ auf $S^{k}$ definiert und $z \in E^{k}$, dann ist die Funktion $F(z)=f(z /|z|)$ auf ganz 
$E^{k}$ außer $z=0$ definiert. Der operator $\Delta$ wird dann definiert durch

$$
\Delta f(x)=|z|^{2} \nabla^{2} F(z),
$$

falls $F(z)$ zweimal differenzierbar auf $E^{k}$ ist. Man sieht, daß $|z|^{2} \nabla^{2} F(z)$ unabhängig vom Abstand des Punktes $z$ vom Nullpunkt ist. Drückt man $\nabla^{2}$ in Kugelkoordinaten (s. [32, II, S. 233]) aus, dann erhält man eine explizite Gestalt von $\Delta$ (s. [32, II, S. 235]). Auf eine andere Art wird der Operator $\Delta$ in [51, S. 38] eingeführt. Für $Y_{n}(x)$ gilt die bekannte Beziehung (s. z.B. [51, S. 39])

$$
\Delta Y_{n}(x)=-n(n+2 \lambda) Y_{n}(x)
$$

Die Anzahl der linear unabhängigen Kugelfunktionen vom Grade $n$ auf $S^{k}$ ist

$$
H(n, k)=(2 n+k-2) \frac{(n+k-3) !}{n !(k-2) !} .
$$

Ein enger Zusammenhang besteht bekanntlich mit den Gegenbauer (ultrasphärischen) Polynomen $P_{n}^{\lambda}(t)$, die definiert sind durch die erzeugende Funktion

$$
\left(1-2 r t+r^{2}\right)^{-\lambda}=\sum_{n=0}^{\infty} r^{n} P_{n}^{\lambda}(t)
$$

oder

$$
\frac{1-r^{2}}{\left(1-2 r t+r^{2}\right)^{\lambda+1}}=\sum_{n=0}^{\infty} \frac{n+\lambda}{\lambda} r^{n} P_{n}^{\lambda}(t) \quad(-1 \leq t \leq 1)
$$

für $0 \leq r<1$ und $\lambda>-1 / 2, \lambda \neq 0$. Für $\lambda=0$ gilt wegen

$$
\lim _{\lambda \rightarrow 0} \lambda^{-1} P_{n}{ }^{\lambda}(\cos \theta)=\frac{2}{n} \cos n \theta \quad(n \geq 1, t=\cos \theta)
$$

die Formel

$$
\frac{1-r^{2}}{1-2 r t+r^{2}}=1+2 \sum_{n=1}^{\infty} r^{n} \cos n \theta .
$$

Wichtig ist die Abschätzung

$$
\begin{aligned}
\left\|P_{n}^{\lambda}\right\|_{C_{\lambda}} & =\sup _{0 \leq \theta \leq \pi}\left|P_{n}^{\lambda}(\cos \theta)\right| \leq\left|P_{n}^{\lambda}(1)\right|=\left(\begin{array}{c}
n+2 \lambda-1 \\
n
\end{array}\right) \\
& =O\left(n^{2 \lambda-1}\right)
\end{aligned}
$$


Die Gegenbauer-Polynome $\left\{P_{n}{ }^{\lambda}(\cos \theta)\right\}$ bilden ein vollständiges Orthogonalsystem auf $[0, \pi]$ mit der Gewichtsfunktion $(\sin \theta)^{2 \lambda}$. Es gelten die folgenden Sätze und Formeln:

Satz 1.1 (Additionstheorem). Ist $\left\{Y_{n}{ }^{l}(x) ; l=1,2, \cdots, H(n, k)\right\}$ die Menge der $H(n, k)$ linear unabhängigen Kugelfunktionen vom Grade $n$ und $\left\{Y_{n}^{l}(x)\right\}$ orthonormiert auf $S^{k}$, dann gilt für alle $x, y \in S^{k}$

$$
P_{n}^{\lambda}[(x, y)]=\frac{2 \pi^{\lambda+1}}{(n+\lambda) \Gamma(\lambda)} \sum_{l=1}^{H} Y_{n}^{l}(x) Y_{n}^{l}(y) \quad(2 \lambda=k-2) .
$$

Satz 1.2 (Funk-Hecke). Ist $f \in \mathbb{L}_{\lambda}{ }^{1}$, dann gilt für alle $n=0,1, \cdots$

$$
\begin{aligned}
& \int_{S^{k}} f[(x, y)] Y_{n}(x) d s(x) \\
& \quad=Y_{n}(y) \frac{(4 \pi)^{\lambda} \Gamma(\lambda) \Gamma(n+1)}{(n+2 \lambda)} \int_{0}^{\pi} f(\theta) P_{n}^{\lambda}(\cos \theta)(\sin \theta)^{2 \lambda} d \theta .
\end{aligned}
$$

Für $n=0$ gilt speziell (mit Formel 15 in [32, I, S. 5])

$$
\int_{S^{k}} f[(x, y)] d s(x)=\Omega_{k-1} \int_{0}^{\pi} f(\theta)(\sin \theta)^{2 \lambda} d \theta .
$$

Mit Hilfe des Additionstheorems folgt hieraus für $x, y, u \in S^{k}$ die Formel

$$
\begin{aligned}
& \int_{S^{k}} f[(x, y)] P_{n}[(y, u)] d s(y) \\
& \quad=P_{n}[(x, u)] \frac{(4 \pi)^{\lambda} \Gamma(n+1) \Gamma(\lambda)}{\Gamma(n+2 \lambda)} \int_{0}^{\pi} f(\theta) P_{n}^{\lambda}(\cos \theta)(\sin \theta)^{2 \lambda} d \theta .
\end{aligned}
$$

Die Menge $\left\{Y_{n}{ }^{l}(x), 1=1,2, \cdots, H(n, k) ; n=0,1,2, \cdots\right\}$ bildet ein vollständiges Orthogonalsystem auf $S^{k}$ in den $\mathbb{C}\left(S^{k}\right)$ und $\boldsymbol{L}^{p}\left(S^{k}\right), 1 \leq p<\infty$. In $\boldsymbol{L}^{2}\left(S^{k}\right)$ gilt außerdem die Parsevalsche Gleichung und der Satz von Riesz-Fischer. Ist $f \in \mathbb{L}^{1}\left(S^{k}\right)$, dann kann man $f$ in eine Reihe von Kugelfunktionen entwickeln, d.h.

$$
S(f ; x) \sim \sum_{n=0}^{\infty} Y_{n}(f ; x),
$$

wobei $Y_{n}(f ; x)$ gegeben ist durch

(1. 11) $Y_{n}(f ; x)=\frac{\Gamma(\lambda)(n+\lambda)}{2 \pi^{\lambda+1}} \int_{S^{k}} P_{n}^{\lambda}[(x, y)] f(y) d s(y) \quad(n=0,1,2, \cdots)$. 
Man nennt $S(f ; x)$ auch die Laplace-Reihe von $f$.

Drückt man den Punkt $y$ auf $S^{k}$ durch Kugelkoordinaten mit den Winkeln $\theta_{1}, \theta_{2}, \cdots, \theta_{k-2}, \varphi$ aus (für die Bezeichnungen siehe [32, II, S. 233]) und ist die Funktion $g(y)$ nur vom Winkel $\theta_{1}\left(=\theta^{\prime}\right)$ abhängig, dann folgt mit Formel (1.10) und $x=(1,0, \cdots, 0), y=$ $\left(\cos \theta^{\prime}, \cdots\right), u=(\cos \theta, \cdots), g(y) \equiv h[(x, y)] \equiv h\left(\cos \theta^{\prime}\right) \equiv f\left(\theta^{\prime}\right)$

$$
\begin{aligned}
Y_{n}(g ; u) & =P_{n}^{\lambda}(\cos \theta) c(n, \lambda) \int_{0}^{\pi} f\left(\theta^{\prime}\right) P_{n}\left(\cos \theta^{\prime}\right)\left(\sin \theta^{\prime}\right)^{2 \lambda} d \theta^{\prime} \\
& =\hat{f}(n) P_{n}^{\lambda}(\cos \theta)
\end{aligned}
$$

mit

$$
c(n, \lambda)=\frac{2^{2 \lambda-1} \Gamma^{2}(\lambda)(n+\lambda) \Gamma(n+1)}{\pi \Gamma(n+2 \lambda)}=\frac{\Gamma(\lambda) \Gamma(2 \lambda)(n+\lambda) \Gamma(n+1)}{\sqrt{\pi} \Gamma(\lambda+1 / 2) \Gamma(n+2 \lambda)} .
$$

Die zonale Funktion $g(y)=f\left(\theta^{\prime}\right)$ ist also in eine Reihe von Gegenbauer-Polynomen entwickelbar. Die Zahlen $\hat{f}(n)(n=0,1,2, \cdots)$ heißen die Gegenbauer-Koeffizienten der Funktion $f$.

Diese Eigenschaften der Kugelfunktionen findet man z.B. in [32, II, Kap. XI], [51] oder [26, Kap. 6], den Satz von Funk und Hecke in dieser Formulierung auch in [63]. Eine ausführliche Darstellung für $k=3$ gibt $G$. Sansone [62].

Mit diesen Hilfsmitteln kann man auf $S^{k}$ den Begriff der Faltung einer Funktion auf $S^{k}$ mit einer Funktion auf $[0, \pi]$ (also einer zonalen Funktion) definieren, wie es zuerst A. P. Calderón und $A$. Zygmund [27, S. 218] 1955 getan haben. Die Faltung zweier zonaler Funktionen hat S. Bochner [8] eingeführt und I. I. Hirschman, Jr. [38] hat sie ausführlich behandelt.

Definition. Ist $f \in \boldsymbol{L}^{1}\left(S^{k}\right)$ und $g \in \boldsymbol{L}_{\lambda}{ }^{1}, 2 \lambda=k-2$, dann heißt die Funktion $h=f * g$, definiert durch

$$
h(x)=\frac{1}{\Omega_{k}} \int_{s^{k}} f(y) g[(x, y)] d s(y)
$$

die Faltung der Funktionen $f$ und $g$.

Sie hat die folgenden Eigenschaften.

Satz 1. 3. a) Ist $f \in \boldsymbol{L}^{p}\left(S^{k}\right)$ und $g \in \boldsymbol{L}_{\lambda}^{q}, 1 \leq p, q \leq \infty$, dann existiert die Faltung $f * g$ fast überall auf $S^{k}$ und es gilt die Youngsche 


\section{Ungleichung}

$$
\|f * g\|_{r} \leq\|f\|_{p} \cdot\|g\|_{q, \lambda} \quad\left(\frac{1}{r}=\frac{1}{q}+\frac{1}{p}-1 \geq 0\right) .
$$

Speziell gilt

$$
\begin{array}{lr}
\|f * g\|_{p} \leq\|f\|_{p}\|g\|_{1, \lambda} & (q=1), \\
\|f * g\|_{q} \leq\|f\|_{1}\|g\|_{q, \lambda} & (p=1) .
\end{array}
$$

b) Ist $f \in \boldsymbol{C}\left(S^{k}\right)$ und $g \in \boldsymbol{L}_{\lambda}{ }^{1}$, dann existiert die Faltung $f * g$ überall auf $S^{k}$, ist wieder aus $\boldsymbol{C}\left(S^{k}\right)$, und es gilt

$$
\|f * g\|_{\boldsymbol{C}} \leq\|f\|_{\boldsymbol{C}}\|g\|_{1, \lambda} .
$$

c) Die Entwicklung nach Kugelfunktionen von $f * g$ hat die Form

$$
Y_{n}(f * g ; x)=\frac{\lambda}{n+\lambda} \hat{g}(n) Y_{n}(f ; x),
$$

wobei $\hat{g}(n)$ und $Y_{n}(f ; x)$ durch (1.12) und (1.11) gegeben sind. Falls $f$ eine zonale Funktion ist, so auch $f * g$.

Dieser Faltungsbegriff wurde kürzlich ausführlich von C. F. Dunkl [30] behandelt. Dort wird u.a. die Faltung einer Funktion mit einem zonalen $\mathrm{Maß}$ bzw. einer zonalen Funktion mit einem $\mathrm{Ma} \beta$ auf $S^{k}$ eingeführt. Ist $\boldsymbol{M}\left(S^{k}\right)$ der Raum aller endlichen, regulären Borelmaße auf $S^{k}$ mit der Norm

$$
\|\mu\|_{M} \equiv \frac{1}{\Omega_{k}} \int_{S^{k}}|d \mu(x)|,
$$

dann kann man jedem $\mathrm{Maß} \mu \in \boldsymbol{M}\left(S^{k}\right)$ eine Reihe von Kugelfunktionen zuordnen, die durch

$$
S\left(d_{\mu} ; x\right) \sim \sum_{n=0}^{\infty} Y_{n}\left(d_{\mu} ; x\right)
$$

mit

(1. 16) $\quad Y_{n}\left(d_{\mu} ; x\right)=\frac{\Gamma(\lambda)(n+\lambda)}{2 \pi^{\lambda+1}} \int_{S^{k}} P_{n}^{\lambda}[(x, y)] d \mu(y) \quad(n=0,1, \cdots)$ gegeben ist. Für die Faltung eines solchen Maßes $\mu$ mit einer zonalen Funktion hat man

Satz 1. 4. Es sei $f \in \boldsymbol{L}_{\lambda}{ }^{p}, 1 \leq p \leq \infty$, oder aus $\boldsymbol{C}_{\lambda}$ und $\mu \in \boldsymbol{M}\left(S^{k}\right)$, dann definiert das Integral 


$$
(f * d \mu)(x)=\frac{1}{\Omega_{k}} \int_{S^{k}} f[(x, y)] d \mu(y)
$$

ein Element aus $\boldsymbol{L}^{p}\left(S^{k}\right)$ bzw. $\boldsymbol{C}\left(S^{k}\right)$ mit $\left\|f * d_{\mu}\right\|_{p} \leq\|f\|_{p, \lambda}\|\mu\|_{M}$ und

$$
Y_{n}(f * d \mu ; x)=(\lambda /(n+\lambda)) \hat{f}(n) Y_{n}\left(d_{\mu} ; x\right) .
$$

Ist außerdem $\mu$ zonal, so auch $f * d \mu$.

Es bleibt noch die Faltung einer Funktion auf $S^{k}$ mit einem zonalen Maß zu definieren. Nach [30] geschieht dies wie folgt. Das Maß ist zonal, also invariant bei Drehungen um den Nordpol $p \in S^{k}$. Wir drehen nun den Punkt $p$ auf $S^{k}$ so, daß das dadurch definierte $\mathrm{Maß} \varphi_{x} \mu$ invariant bei Drehungen ist, die den Punkt $x$ fest lassen (für Einzelheiten siehe [30, S. 252]). Damit kommen wir zu der Definition einer Faltung mit einem zonalen Maß.

Satz 1.5. Es sei $f \in L^{p}\left(S^{k}\right), 1 \leq p \leq \infty$, oder $\boldsymbol{C}\left(S^{k}\right)$ und $\mu \in \boldsymbol{M}_{\lambda}$, dann definiert die Formel

$$
\left(f * d_{\mu}\right)(x)=\frac{1}{\Omega_{k}} \int_{S^{k}} f(y) d \varphi_{x} \mu(y)
$$

ein Element aus $\boldsymbol{L}^{p}\left(S^{k}\right)$ bzw. $\boldsymbol{C}\left(S^{k}\right)$ mit $\|f * d \mu\|_{p} \leq\|f\|_{p}\|\mu\|_{\boldsymbol{M}_{\lambda}}$ bzw. $\|f * d \mu\|_{\boldsymbol{c}} \leq\|f\|_{\boldsymbol{C}}\|\mu\|_{M_{\lambda}}$. Außerdem gilt

$$
Y_{n}(f * d \mu ; x)=(\lambda /(n+\lambda)) \stackrel{\vee}{\mu}(n) Y_{n}(f ; x) \quad(n=0,1, \cdots)
$$

mit den Gegenbauer-Stieltjes-Koeffizienten

$$
\stackrel{\vee}{\mu}(n)=c(n, \lambda) \int_{0}^{\pi} P_{n}^{\lambda}(\cos \theta) d \mu(\theta)
$$

(siehe [30, S. 252 u. 254]).

Bemerkung. Faltet man eine Funktion $g \in \boldsymbol{L}_{\lambda}^{1}, 2 \lambda=k-2$, mit einer Funktion $f \in \boldsymbol{X}$, dann folgt aus der Definition der Faltung und Ungleichung (1.14), daß die Faltung eine lineare, beschränkte Operation von $\boldsymbol{X}$ in sich bildet.

Die Faltung führt nun zum Begriff eines singulären Integrals. Es sei $f \in \boldsymbol{X}$ und $K_{\rho} \in \boldsymbol{L}_{\lambda}{ }^{1}, \rho>0$, mit den Eigenschaften

$$
c(0, \lambda) \int_{S^{k}} K_{\rho}(\cos \theta)(\sin \theta)^{2 \lambda} d \theta=1
$$




$$
c(0, \lambda) \int_{S^{k}}\left|K_{\rho}(\cos \theta)\right|(\sin \theta)^{2 \lambda} d \theta \leq M
$$

(gleichmäßig in $\rho>0$ mit einer Konstanten $M \geq 1$ );

$$
\lim _{\rho \rightarrow \infty} \sup _{\delta \leq \theta \leq \pi}\left|K_{\rho}(\cos \theta)\right|=0 \quad \text { (für jedes feste } \delta>0 \text { ). }
$$

Dann bildet

$$
I_{\rho}(f ; x)=\frac{1}{\Omega_{k}} \int_{S^{k}} f(y) K_{\rho}[(x, y)] d s(y) \quad(\rho>0)^{1)}
$$

ein singuläres Integral mit dem Kern $K_{\rho}$ und Parameter $\rho>0$. $c(0, \lambda)$ ist in (1.12) gegeben und $c(0, \lambda)=\Omega_{k-1} / \Omega_{k}$.

Das singuläre Integral $I_{\rho}(f ; x)$ hat die folgende Eigenschaften.

Satz 1.6. Ist das singuläre Integral $I_{\rho}(f ; x)$ vom Typ (1.23) und genügt sein Kern den Bedingungen (1.20), (1.21) und (1.22), dann gilt für alle $f \in \boldsymbol{X}$

$$
\begin{aligned}
& \left\|I_{\rho} f\right\| \leq M\|f\| ; \\
& \lim _{\rho \rightarrow \infty}\left\|I_{\rho} f-f\right\|=0 .
\end{aligned}
$$

Der Beweis verläuft wie üblich. (1.24) folgt direkt aus Satz 1.3 und (1.21). Ist $D(x, h)=\left\{y ; y \in S^{k},(x, y) \geq \cos h, 0<h<\pi\right\}$, dann ist

$$
\begin{aligned}
I_{\rho}(f ; x)-f(x)= & \frac{1}{\Omega_{k}} \int_{s^{k}-D(x, h)} K_{\rho}[(x, y)][f(y)-f(x)] d s(y) \\
& \quad+\frac{1}{\Omega_{k}} \int_{D(x, h)} K_{\rho}[(x, y)][f(y)-f(x)] d s(y)=I_{1}+I_{2} .
\end{aligned}
$$

Nach der Hölder-Minkowski-Ungleichung und (1.9) ist

$$
|| I_{1}\left\|\leq \frac{2}{\Omega_{k}}\right\| f\left\|\Omega_{k-1} \int_{h}^{\pi}\left|K_{\rho}(\cos \theta)\right|(\sin \theta)^{2 \lambda} d \theta \leq 2|| f\right\| \sup _{h \leq \theta \leq \pi}\left|K_{\rho}(\cos \theta)\right|<\varepsilon,
$$

falls $\rho>\rho_{0}(\varepsilon), \varepsilon>0$ vorgegeben.

Ebenfalls nach der Hölder-Minkowski-Ungleichung und der Stetigkeit bzw. Stetigkeit im Mittel von $f$ gilt

$$
\left\|I_{2}\right\| \leq \frac{1}{\Omega_{k}} \int_{D(x, h)}\left|K_{\rho}[(x, y)]\right|\|f(y)-f(x)\| d s(y)<\varepsilon \cdot M, \quad \text { falls } \quad h<\delta .
$$

1) Wir schreiben der Einfachheit halber oft $I_{\rho} f$ an Stelle von $I_{\rho}(f, X)$. 
Also $\left\|I_{\rho} f-f\right\| \leq\left\|I_{1}\right\|+\| I_{2} \mid<(M+1) \varepsilon$, falls $\rho>\rho_{0}(\varepsilon)$, womit (1.25) bewiesen ist.

Beispiele von singulären Integralen werden im nächsten $\mathrm{Ab}$ schnitt vorkommen, in dem die Konvergenz und Summation in der Norm von Reihen von Kugelfunktionen behandelt wird.

\section{Konvergenz und Summation von Kugelfunktionen}

\section{1. Allgemeines.}

Entwickelt man eine Funktion $f \in \boldsymbol{C}\left(S^{k}\right)$ in eine Reihe von Kugelfunktionen und bezeichnet man die Teilsummen der Reihe mit

$$
S_{N}(f ; x)=\sum_{n=0}^{N} Y_{n}(f ; x)
$$

dann stellt sich die Frage nach der Konvergenz von $S_{N} f$ gegen $f$ für $N \rightarrow \infty$. Da die Teilsummen $S_{N}$ eine Folge von beschränkten linearen Operatoren bilden, und da für ihre Normen $\left\|S_{N}\right\|$ (d.h. Lebesgue-Konstanten), im Raum $\mathbb{C}\left(S^{k}\right)$ gilt $\lim _{N \rightarrow \infty}\left\|S_{N}\right\|=+\infty$ ([28], [40]), folgt nach dem Satz von Banach-Steinhaus die Existenz einer Funktion $f_{0} \in C\left(S^{k}\right)$, für die die zugeordnete Reeihe $S\left(f_{0} ; x\right)$ an mindestens einem Punkte $x_{0} \in S^{k}$ divergiert. Die Teilsummen $S_{N}(f ; x)$ konvergieren also nicht in der $\boldsymbol{C}$-Norm gegen $f(x)$.

Im Raume $\mathbb{L}^{2}\left(S^{k}\right)$ folgt die Normkonvergenz der Teilsummen nach dem Satz von Riesz-Fischer. Komplizierter wird es in den $\boldsymbol{L}^{p}$-Räumen für $1 \leq p<\infty, p \neq 2$. Für zonale Funktionen, also Entwicklungen nach Gegenbauer-Polynomen hat man Normkonvergenz für $(2 \lambda+1) /(\lambda+1)<p<(2 \lambda+1) / \lambda$, siehe [59]. Das Ergebnis ist falsch für die anderen Werte von $p$ [56]. Hieraus kann man schließen, da $2 \lambda=k-2$, daß keine Normkonvergenz von (2.1.1) für $p \geq 2+(2 /(k-2))$ und $p \leq 2-(2 / k)$ vorliegt. Die Normkonvergenz für $p$ mit $2-(2 / k)<p<2+(2 /(k-2))$ wird vermutet (s. [3, S. 812]).

Man kann nun Konvergenzfaktoren benutzen und die Summierbarkeit der Reihe $S(f ; x)$ untersuchen. Als erstes sollen die Cesàro-Mittel $(C, \alpha)$ einer Reihe definiert werden. Die Summen

$$
\sigma_{N}^{\alpha}(f ; x) \equiv\left(A_{N}^{\alpha}\right)^{-1} \sum_{n=0}^{N} A_{N-n}^{\alpha} Y_{n}(f ; x)
$$


mit den verallgemeinerten Binomialkoeffizienten

$$
A_{n}^{\alpha}=\left(\begin{array}{c}
n+\alpha \\
n
\end{array}\right)=\frac{(\alpha+1) \cdots(\alpha+n)}{n !} \quad(\alpha \geq 0),
$$

heißen $N$-te $(C, \alpha)$-Mittel der Reihe $S(f ; x)$. Ist $\alpha=1$, so werden die $\sigma_{N}^{1}$ die arithmetischen oder Fejérschen Mittel der Reihe $S(f ; x)$ genannt. Mit (1.11) kann man $\sigma_{N}^{\alpha}$ in der Gestalt (1.13) schreiben, d.h.

$$
\sigma_{N}^{\alpha}(f ; x)=\frac{1}{\Omega_{k}} \int_{S^{k}} K_{N}^{\alpha}[(x, y)] f(y) d s(y)
$$

mit dem Kern

$$
K_{N}^{\alpha}(t)=\left(A_{N}^{\alpha}\right)^{-1} \sum_{n=0}^{N} A_{N-n}^{\alpha} \frac{n+\lambda}{\lambda} P_{n}^{\lambda}(t) \quad(-1 \leq t \leq 1) .
$$

Es gelten die folgenden Eigenschaften.

Satz 2.1.1. Ist $f \in X$, sind $\sigma_{N}^{\alpha}(f ; x)$ und $K_{n}^{\alpha}(t)$ durch (2.1.2) bzw. (2.1.5) gegeben, dann gilt

$$
\begin{array}{lr}
\left\|K_{N}^{\alpha}\right\|_{1, \lambda}=\frac{\Omega_{k-1}}{\Omega_{k}} \int_{0}^{\pi}\left|K_{N}^{\alpha}(\cos \theta)\right|(\sin \theta)^{2 \lambda} d \theta \leq M_{\infty} \\
(\alpha>\lambda ; N=0,1, \cdots) ; \\
K_{N}^{\alpha}(t) \geq 0 & (-1 \leq t \leq 1 ; \alpha \geq 2 \lambda+1=k-1) ; \\
\lim _{N \rightarrow \infty}\left\|\sigma_{N}^{\alpha} f-f\right\|=0 & (f \in \boldsymbol{X} ; \alpha>\lambda) .
\end{array}
$$

(2.1.8) wurde in [28] und [40] bewiesen. Dort wurde auch gezeigt, daß (2.1.6) für $\alpha \leq \lambda$ nicht mehr gültig ist. (2.1.7) wurde ebenfalls in [40, S. 179] bewiesen. Die Reziehung (2.1.8) folgt aus (2.1.6) mit dem Satz von Banach-Steinhaus.

Als nächstes soll das Summationsverfahren von Abel-Poisson auf $S^{k}$ betrachtet werden. Es ist gegeben durch

$$
A_{r}(f ; x)=\sum_{n=0}^{\infty} r^{n} Y_{n}(f ; x)
$$

oder wegen (1.4) und (1.11) in der Form

$$
A_{r}(f ; x)=\frac{1}{\Omega_{k}} \int_{s^{k}} \frac{1-r^{2}}{\left(1-2 r(x, y)+r^{2}\right)^{\lambda+1}} f(y) d s(y) .
$$

Setzt man $r \cdot x=z$ und $A(z) \equiv A_{r}(f ; x)$, dann ist $A(z)$ Lösung der Laplace-Gleichung 


$$
\nabla^{2} A(z)=0
$$

$(|z|<1)$

für das Innere der Einheitskugel in $E^{k}$, wobei $A$ eine dort beschränkte Funktion ist, die der Randbedingung

$$
\lim _{r \rightarrow 1-} A(z)=f(x) \quad(z=r x,|z|=r)
$$

genügt, wobei der Grenzwert in einem bestimmten Sinne zu verstehen ist (siehe z.B. [57, S. 9]). Es gilt

Satz 2.1.2. Ist $f \in \boldsymbol{X}$; dann gilt
a) $A_{r}(f ; x)$ ist harmonisch, d.h. $\nabla^{2} A(z)=0$, für $|z|<1$;
b) ist $f \geq 0$ auf $S^{k}$, so ist auch $A_{r}(f ; x) \geq 0$;
c) weiter gilt

$$
\left\|A_{r} f\right\| \leq\|f\|
$$

und

$$
\lim _{r \rightarrow 1-}\left\|A_{r} f-f\right\|=0 \text {. }
$$

Beweis : Teil a) folgt daraus, daß $r^{n} Y_{n}(f ; x)=Q_{n}(z)$ harmonisch für $|z|<1$ und alle $n=0,1,2, \cdots$ ist, und daß die Reihe (2.1.9) gliedweise differenziert werden kann. Dies folgt aus

$$
\left\|Y_{n}(f ; x)\right\|_{c} \leq \frac{\Gamma(\lambda)(n+\lambda)}{2 \pi^{\lambda+1}}\left\|P_{n}{ }^{\lambda}\right\|_{c_{\lambda}}\|f\|_{1}=O\left(n^{2 \lambda}\right) \quad(n \rightarrow \infty)
$$

wegen (1.6).

(2.1.13) ergibt sich aus der Positivität des Kerns

$$
p_{r}(t)=\frac{1-r^{2}}{\left(1-2 r t+r^{2}\right)^{\lambda+1}} \geq 0, \quad(-1 \leq t \leq 1) .
$$

Wegen (1.4), (1.9) und der Orthogonalität der Gegenbauer-Polynome gilt nämlich

$$
\frac{1}{\Omega_{k}} \int_{S^{k}} p_{r}[(x, y)] d s(y)=1 \quad(0 \leq r<1) .
$$

Um (2.1.14) zu beweisen, kann man Satz 1.6 anwenden. Es gilt nämlich

$$
\lim _{r \rightarrow 1-} \sup _{\delta \leq \theta \leq \pi}\left|p_{r}(\cos \theta)\right| \leq \lim _{r \rightarrow 1-} \frac{1-r^{2}}{\left(1-2 r \cos \delta+r^{2}\right)^{\lambda+1}}=0 \quad(\delta>0),
$$


und daher ist $A_{r}(f ; x)$ ein singuläres Integral vom Typ (1.23) mit den Eigenschaften (1.20), (1.21) und (1.22), wenn man $\rho=\log (1 / 1-r)$, $r \rightarrow 1$ - setzt. Damit ist der Satz bewiesen.

Die Bedingung b) in Satz 2.1.2 sagt aus, daß $A_{r}$ für jedes $r$ mit $0 \leq r<1$ ein positiver Operator ist. Positive Operateren auf $X$ sind wie folgt definiert.

Definition. Ein Operator $T$ heißt positiv, falls aus $f(x) \geq 0$ für jedes $x \in S^{k}$ folgt $T(f ; x) \geq 0$ für $x \in S^{k}$.

Positive Operatoren sind von besonderem Interesse, da für sie einfache Konvergenzsätze gelten. Die folgende Menge $\mathcal{I}\left(S^{k}\right)$ von stetigen Funktionen auf $S^{k}$ ist gegeben durch

$$
\Re\left(S^{k}\right)=\left\{1, e_{j}(x), 1 \leq j \leq k\right\},
$$

wobei $e_{j}(x), 1 \leq j \leq k$, die Komponenten des Einheitsvektors $x \in E^{k}$ in Kugelkoordinaten sind.

Als Anwendung eines Satzes über positive Operatoren für stetige Funktionen, die auf kompakten Hausdorffräumen definiert sind [47, S. 7], erhält man

Satz 2.1.3. Es sei $\left\{T_{\rho}, 0<\rho<\infty\right\}$ eine Menge von positiven Operatoren von $\mathrm{C}\left(S^{k}\right)$ in sich. Aus der Bedingung

folgt

$$
\lim _{\rho \rightarrow \infty} \| T_{\rho} f-f \mid !=0
$$

$$
\lim _{\rho \rightarrow \infty}\left\|T_{\rho} f-f\right\|=0
$$$$
\left(f \in C\left(S^{k}\right)\right) .
$$

Bemerkung. Im Falle $k=2$ hat man den Raum der auf der Einheitskreislinie in $E^{2}$ definierten stetigen Funktionen und $\mathscr{\eta}\left(S^{2}\right)$ ist die Menge der Funktionen $1, \sin \varphi, \cos \varphi, 0 \leq \varphi<2 \pi$. Dies ist der bekannte Satz von H. Bohman [10] und P. P. Korovkin [41].

\subsection{Sätze vom Bernsteinschen und Jacksonschen Typ.}

Bei der Approximation von Funktionen durch Approximationsverfahren taucht die Frage auf, mit welcher Genauigkeit eine gegebene Funktion durch ein solches Verfahren (z.B. die Teilsummen einer Reihenentwicklung, Summationsverfahren einer solchen Reihe oder 
ein singuläres Integral) approximiert werden kann. Man kann von gewissen Eigenschaften der Funktion (z.B. einer Lipschitzbedingung) auf die Approximationsordnung durch das Verfahren schließen (sogenannte direkte Sätze) oder von der Approximationsordnung auf Eigenschaften der Funktion (indirekte Sätze oder Umkehrsätze).

Wir definieren den Stetigkeitsmodul einer Funktion $f \in \boldsymbol{C}\left(S^{k}\right)$ durch

$$
\omega(\delta)=\omega(f ; \delta)=\sup _{|x-y| \leq \delta}|f(x)-f(y)|,
$$

wobei $|x-y|=(x-y, x-y)^{1 / 2}$ der euklidische Abstand zwischen $x$ und $y$ in $E^{k}$ ist.

Man sagt, $f$ erfüllt eine Lipschitzbedingung der Ordnung $\alpha$, $0<\alpha \leq 1$, falls

$$
\omega(f ; \delta) \leq M \delta^{a} \quad(M \text { positive Konstante } ; \delta \rightarrow 0+)
$$

ist, und schreibt $f \in \operatorname{Lip} \alpha$. Wir wollen zuerst zwei Sätze über die Approximation durch Polynome angeben. Mit $\mathscr{L}_{n}$ bezeichnen wir die Menge aller Polynome $P_{n}(x)$ vom Grade $\leq n$. Die Polynome bester Approximation $P_{n}{ }^{*}(x)$ der Funktion $f$ sind definiert durch

$$
\left\|f-P_{n}^{*}\right\|_{C}=\inf _{P_{n} \in \mathscr{P}_{n}}\left\|f-P_{n}\right\|_{c} \cdot{ }^{2)}
$$

Ein Analogon zum Satz von Jackson für trigonometrische Polynome ([74, S. 115]) lautet (s. [55, S. 212] o. [60, S. 19]).

Satz 2.2.1. Ist $f \in C\left(S^{k}\right)$ und besitzt den Stetigkeitsmodul $\omega(\delta)$, dann existiert ein Polynom $P_{n} \in \mathscr{Q}_{n}$ mit

$$
\left\|f-P_{n}\right\|_{C} \leq C_{1} \omega\left(\frac{k}{n}\right)
$$

Ist speziell $\omega(\delta) \leq M \delta^{\alpha},(\delta \rightarrow 0+), 0<\alpha \leq 1$, dann folgt $\left\|f-P_{u}\right\|_{\tau} \leq C_{2} n^{-\infty}$ $(n \rightarrow \infty)$.

Eine Umkehrung dazu wurde für trigonometrische Polynome von S. Bernstein ([74, S. 119]) bewiesen. Hier lautet sie (s. [60, S. 33])

2) Die Existenz der Polynome bester Approximation ist gesichert (s. z.B. [47, S. 17]). Sie sind allerdings nicht eindeutig (s. [47, S. 25 , Sec. 2.5 u. S. 34$]$ ). 
Satz 2.2.2. Ist $f \in C\left(S^{k}\right)$ und gilt für die Polynome bester Approximation $\left\{P_{n}{ }^{*}\right\}(n=0,1,2, \cdots)$ und für ein $\alpha$ mit $0<\alpha<1$

$$
\left\|P_{n}^{*}-f\right\|_{C}=O\left(n^{-\infty}\right) \quad(n \rightarrow \infty),
$$

dann folgt $f \in \operatorname{Lip} \alpha$.

In der Approximationstheorie auf $S^{3}$ wurden die Mittel $S_{h}(f ; x)$ untersucht, die in Abschnitt 4.2 durch (4.2.8) definiert sind. Hier $(k=3)$ haben sie die Form

$$
S_{h}(f ; x) \equiv \frac{1}{2 \pi \sin h} \int_{(x, y)=\cos h} f(y) d t(y),
$$

wobei $d t$ das Bogenelement der Kurve $(x, y)=\cos h$ auf $S^{3}$ ist. Diese Definition stammt von W. Rudin [61, S. 288]. Für sie gilt der folgende Satz (s. [43]) :

Satz 2.2.3. Ist $f \in \boldsymbol{C}\left(S^{3}\right)$ und $0<\alpha<2$, dann sind die folgenden Aussagen äquivalent :

a)

$$
\begin{array}{lr}
\sup _{0<h \leq \delta} \mid S_{h} f-f \|_{C}=O\left(\delta^{\infty}\right) & (\delta \rightarrow 0+) ; \\
\left\|P_{n}{ }^{*}-f\right\|_{C}=O\left(n^{-\infty}\right) & (n \rightarrow \infty) .
\end{array}
$$

b)

Ähnliche Aussagen gelten auch für $\alpha \geq 2$ (siehe [31] und [43]). Die Lipschitzbedingung in den Sätzen 2.2.1 und 2.2.2 ist hier durch die Aussage a) ersetzt worden.

Nimmt man an Stelle von Polynomen das singuläre Integral von Abel-Poisson, definiert durch (2.1.9), als Approximationsverfahren, dann gilt ein sehr ähnlicher Satz (s. [58]).

Satz 2.2.4. Eine notwendige und hinreichende Bedingung, damit $f \in \boldsymbol{C}\left(S^{k}\right)$ zur Klasse Lip $\alpha$ für ein festes $\alpha$ mit $0<\alpha<1$ gehört, ist

$$
\| A_{r} f-\left.f\right|_{\left.\right|_{C}}=O\left((1-r)^{\infty}\right) \quad(r \rightarrow 1-) .
$$

Für $S^{2}$ ist dieser Satz auch mit Halbgruppenmethoden in [5] bewiesen worden. In $S^{2}$ ist außerdem der Fall $\alpha=1$ gelöst. Hier gilt $\left\|A_{r} f-f\right\|_{C}=O(1-r)(r \rightarrow 1-)$ genau dann, wenn $\tilde{f} \in \operatorname{Lip} 1([11],[13])$, wobei $\tilde{f}$ die zu $f$ konjugierte Funktion bedeutet. 
Bemerkung 2.2.5. Ersetzt man die Bedingung $O(1-r)$ durch $o(1-r)$, dann folgt, daß die Funktion $f$ konstant ist. Es werden also auch solche Funktionen wie $\sin \varphi$ und $\cos \varphi$ nicht besser als mit der Ordnung $O(1-r)$ approximiert. Diese "optimale” Approximationsordnung durch das Abelsche Summationsverfahren bestimmt eine Klasse von Funktionen. Solche Funktionenklassen auf der Kugel sollen in den nächsten Abschnitten dieser Arbeit untersucht werden. Die meisten bekannten Summationsverfahren besitzen ein entsprechendes Verhalten.

\section{Ein Allgemeiner Saturationssatz}

Wie aus der Bemerkung 2.2.5 zu ersehen ist, kann bei gewissen Summationsverfahren die Approximation nicht über eine bestimmte Ordnung hinaus verbessert werden, wenn auch die Funktion z.B. beliebig oft differenzierbar ist. Diese Ordnung nennt man Saturationsordnung des Verfahrens und die Klasse von Funktionen, die genau mit dieser Ordnung approximiert werden kann, heißt Saturationsklasse oder Favard-Klasse. Diese Begriffe wurden von J. Favard [33] eingeführt.

Definition 3. 1. Es sei $\varphi(u)$ eine positive, nicht wachsende Funktion in $0<u<\infty$ mit $\lim _{u \rightarrow \infty} \varphi(u)=0$. Ist $I_{\rho} f$ ein durch (1.23) gegebenes singuläres Integral und existiert eine Klasse $\mathcal{K} \subset \boldsymbol{X}$ von Funktionen $f$, so daß für $\rho \rightarrow \infty$ gilt :

a) aus $\left\|I_{\rho} f-f\right\|=o(\varphi(\rho))$ folgt $I_{\mathrm{\rho}} f=f$ $(\rho>0)$

b) aus $\left\|I_{\rho} f-f\right\|=O(\varphi(\rho))$ folgt $f \in \mathcal{K}$,

c) für jedes $f \in \mathcal{K}$ gilt $\left\|I_{\rho} f-f\right\|=O(\varphi(\rho))$, dann heißt $I_{\rho}$ saturiert mit der Ordnung $\varphi(\rho)$ und $\mathcal{K}$ die Saturationsklasse.

Es wurde zuerst für periodische, also auf dem Einheitskreis in $E^{2}$ definierte Funktionen für die Cesàro-Mittel von G. Alexits [1], dann für weitere spezielle polynomiale Verfahren von M. Zamansky [72] gelöst. Die ersten allgemeinen Sätze stammen von P. L. Butzer [13], [14], J. Favard [34], H. Buchwalter [11], [12] und G. Sunouchi 
und C. Watari [69], [70]. Eine ausführliche Literaturangabe findet man z.B. in [20].

Es sind nun mehrere allgemeine Methoden zur Bestimmung der Saturationsklasse eingeführt worden. Die Integraltransformationsmethode geht zurück auf $P$. L. Butzer [14] (siehe auch [18]. Mit ihrer Hilfe kann man z.B. das Saturationsproblem bei singulären Integralen für $2 \pi$-periodische Funktionen (die Fourierkoeffizientenmethode) und für Funktionen auf $E^{1}$ (Fouriertransformationsmethode) lösen. Auch für andere Probleme in der Analysis ist diese Methode geeignet [15]. Die Fouriertransformationsmethode kann nicht in den Räumen $\mathbb{E}^{p}\left(E^{1}\right), p>2$, angewandt werden, da dort keine Fouriertransformation im klassischen Sinn definiert werden kann. Für alle Funktionenräume $\mathbb{L}^{p}\left(E^{1}\right), 1 \leq p<\infty$, (und für andere) erweist sich die Funktional- oder Distributionenmethode als nützlich, die in einer Arbeit von E. Görlich und R. J. Nessel [36] behandelt und ausführlich erörtert wurde.

Bildet das Summationsverfahren eine stark stetige Halbgruppe von Operatoren der Klasse $\left(C_{0}\right)$ (im Sinne von E. Hille-R. S. Phillips [37]; für die Definition siehe auch Abschnitt 4.1 dieser Arbeit), dann gibt es eine weitere Möglichkeit, das Saturationsproblem zu behandeln. In [13] hat P. L. Butzer gezeigt, daß Halbgruppen von Operatoren (auf Banachräumen) ein Saturationsverhalten haben, und die Saturationsklasse für reflexive Räume bestimmt. Für den dualen Fall siehe $K$. de Leeuw [46]. Eine ausführliche Behandlung findet man in [19].

Hier soll nun das Saturationsproblem für singuläre Integrale auf auf $S^{k}(k \geq 3)$ untersucht werden. Im Falle zonaler Funktionen auf $S^{3}$ (Entwicklungen nach Legendre-Polynomen) wurde das Problem in [16], [18] angeschnitten. Für das singuläre Integral von Abel-Poisson auf $S^{k}, k \geq 3$, wurden vor allem indirekte Sätze mit Hilfe der Transformationsmethode in [21] angegeben.

An die Stelle der Fourierkoeffizienten tritt in [21] die Folge der Kugelfunktionen $\left\{Y_{n}(f ; x), n=0,1,2, \cdots\right\}$. Diese Methode wird hier zum Beweis eines allgemeinen indirekten Satzes für singuläre 
Integrale auf $S^{k}$ benutzt. Der direkte Satz, der für jedes Beispiel getrennt verifiziert wird, folgt in mehreren Fällen mit Hilfe der Halbgruppenmethode. Die Saturationsklasse $\mathcal{K}$ wird hier durch die Glieder $Y_{n}(f ; x)$ der Laplace-Reihe von $f$ charakterisiert. Für die auftretenden Funktionenklassen wird zuerst eine Definition gegeben. $\boldsymbol{X}$ ist dabei immer einer der Räume $\mathbb{L}^{p}\left(S^{k}\right), 1 \leq p<\infty$ oder $\boldsymbol{C}\left(S^{k}\right)$.

Definition 3. 2. $\psi(n)$ ist eine Funktion, die auf den nichtnegativen ganzen Zahlen definiert ist, und höchstens für $n=0$ verschwindet. Die Klassen $\mathscr{H}(\boldsymbol{X}, \psi(n))$ in $\boldsymbol{X}$ sind gegeben durch

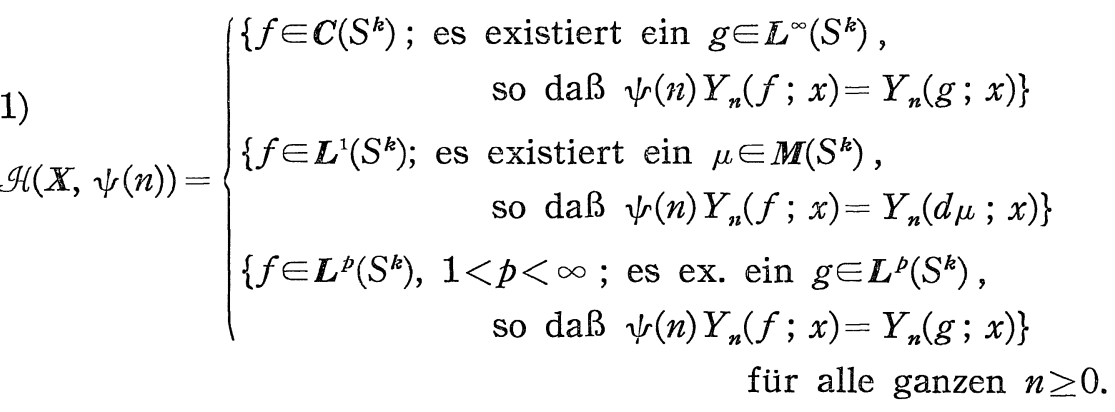

Hiermit soll nun ein Satz bewiesen werden, der mit den Teilen a) und b) der Definition 3.1 in Beziehung steht. Der Beweis verläuft entsprechend dem periodischen Fall (vgl. auch [23, S. 523-527] und [54]). An den Kern $K_{\rho}$ des singulären Integrals wird noch die folgende Bedingung gestellt. Es existiert eine Funktion $\psi(n)$, wie oben definiert, so daß für alle ganzen $n \geq 0$ gilt

$$
\lim _{\rho \rightarrow \infty} \frac{\frac{\lambda}{n+\lambda} \hat{K}_{\rho}(n)-1}{\varphi(\rho)}=\psi(n),
$$

wobei $\hat{K}_{\rho}(n)$ durch (1.12) gegeben ist und $\varphi(\rho)$ in der Definition 3.1 erklärt wurde.

Wir bezeichnen (3.2) als Favard-Bedingung. J. Favard [34] hat als erster für $S^{2}$ gesehen, daß die Kenntnis des Verhaltens von $\left|(\lambda /(n+\lambda)) \hat{K}_{p}(n)-1\right|$ für feste $n$ und $\rho \rightarrow \infty$ ausreicht, um die Existenz der Saturationsklasse $\mathrm{zu}$ zeigen und die zugehörige Saturationsordnung zu bestimmen (siehe dazu auch [11]). Bedingungen der Form (3.2) an einen Kern $K_{\rho}$ wurden zuerst von $H$. Buchwalter 
[11], G. Sunouchi und C. Watari $[70, I]$ und P. L. Butzer [14] gestellt.

Bemerkung. Erfüllt ein singuläres Integral vom Typ (1.23) die Bedingung (1.20), (1.21) und an Stelle von (1.22) die Beziehung (3.2), dann folgt daraus wieder die Aussage (1.25) von Satz 1.6. Aus (1.21) ergibt sich nämlich die gleichmäßige Beschränktheit der Normen der Operatoren $I_{\rho}$ bezüglich $\rho$ (d.h. (1.24)). In (3.2) ist die Aussage

$$
\lim _{\rho \rightarrow \infty}(\lambda /(n+\lambda)) \hat{K}_{\rho}(n)=1
$$

enthalten und es ist $\hat{K}_{\rho}(0)=1$ wegen (1.20). Daraus folgt die Normkonvergenz von $I_{\rho} f$ gegen $f, \rho \rightarrow \infty$, für jedes Polynom $P_{n}(x)$ (die Laplaceentwicklung von $I_{\rho} P_{n}$ besitzt nur endlich viele Glieder). Da die Menge der Polynome dicht in $\boldsymbol{X}$ ist, folgt (1.25) für alle $f \in \boldsymbol{X}$ nach dem Satz von Banach-Steinhaus.

Dies ist in den Fällen wichtig, in denen die Bedingung (1.22) an den Kern $K_{\rho}$ schwerer nachzuweisen ist als die Eigenschaft (3.2). Es wird nun der allgemeine indirekte Satz bewiesen.

Satz 3.1. Ist $f \in \boldsymbol{X}$, ein singuläres Integral $I_{\rho} f$ durch (1.23) gegeben, und erfüllt der Kern $K_{\mathrm{p}}$ die Bedingungen (1.20), (1.21) und (3.2), dann gilt :

a) Existiert eine Funktion $g \in \boldsymbol{X}$, so daß

$$
\lim _{\rho \rightarrow \infty}\left\|\frac{1}{\varphi(\rho)}\left\{I_{\rho} f-f\right\}-g\right\|=0
$$

ist, dann folgt

$$
\psi(n) Y_{n}(f ; x)=Y_{n}(g ; x) \quad(n=0,1,2, \cdots) .
$$

Ist speziell $g=0$ in $\boldsymbol{X}$, dann folgt $f=$ const. in $\boldsymbol{X}$.

b) Aus

$$
\left\|I_{\rho} f-f\right\|=O(\varphi(\rho))
$$

folgt $f \in \mathcal{H}(\boldsymbol{X}, \psi(n))$.

Beweis: a) Für die Glieder der Laplace-Reihe des Ausdrucks in der Norm gilt mit dem Faltungssatz 1.3 


$$
Y_{n}\left\{\frac{1}{\varphi(\rho)}\left[I_{\rho} f-f\right]-g ; x\right\}=\frac{\frac{\lambda}{n+\lambda} \hat{K}_{\rho}(n)-1}{\varphi(\rho)} Y_{n}(f ; x)-Y_{n}(g ; x) .
$$

Außerdem folgt

$$
\begin{aligned}
\left\|Y_{n}\left\{\frac{1}{\varphi(\rho)}\left[I_{\rho} f-f\right]-g\right\}\right\|_{C} & \leq \frac{n+\lambda}{\lambda}\left\|P_{n}{ }^{\lambda}\right\|_{C}\left\|\frac{1}{\varphi(\rho)}\left[I_{\rho} f-f\right]-g\right\| \\
& \leq \frac{n+\lambda}{\lambda}\left(\begin{array}{c}
n+2 \lambda-1 \\
n
\end{array}\right)\left\|\frac{1}{\varphi(\rho)}\left[I_{\rho} f-f\right]-g\right\|,
\end{aligned}
$$

wobei die rechte Seite nach Voraussetzung für $\rho \rightarrow \infty$ gegen Null konvergiert. Also ist wegen (3.2)

$$
\psi(n) Y_{n}(f ; x)=Y_{n}(g ; x) \quad(n=0,12, \cdots) .
$$

b) Hicr benutzt man wie in [21] den Darstellungssatz für stetige, lineare Funktionale auf den Räumen $\boldsymbol{C}\left(S^{k}\right)$ und $\boldsymbol{L}^{q}\left(S^{k}\right)$ [48, S. 211, 218], welcher für $\boldsymbol{L}^{q}\left(S^{k}\right)$ und $1 \leq q<\infty$ die folgende Form hat: Jedes auf $\boldsymbol{L}^{q}\left(S^{k}\right)$ definierte beschränkte, lineare Funktional $F$ läßt sich in der Form

$$
F(h)=\frac{1}{\Omega_{k}} \int_{S^{k}} f(x) \cdot h(x) d s(x) \quad\left(h \in \mathbb{L}^{q}\left(S^{k}\right)\right)
$$

darstellen, wobei die Funktion $f \in \mathbb{L}^{p}\left(S^{k}\right)$ durch das Funktional $F$ eindeutig bestimmt ist. Umgekehrt ist jedes durch (3.5) definierte Funktional auf $\mathbb{L}^{q}\left(S^{k}\right)$ ein stetiges, lineares Funktional. Für die Norm von $F$ gilt

$$
\|F\|=\|f\|_{p} \quad\left(\frac{1}{p}+\frac{1}{q}=1 ; q=1, p=\infty\right) .
$$

Ist nun $1<p<\infty$, dann ist nach Voraussetzung (3.4)

$$
[\varphi(\rho)]^{-1}\left\|I_{\rho} f-f\right\|_{p}=O(1)
$$$$
(\rho \rightarrow \infty)
$$

für ein festes $f \in \boldsymbol{L}^{p}\left(S^{k}\right)$. Ist $f \in \boldsymbol{C}\left(S^{k}\right)$, dann gilt nach (3.4) auch (3.7) für $p=\infty$. Also ist

$$
F_{\rho}(h)=\frac{1}{\Omega_{k} \varphi(\rho)} \int_{s^{k}}\left\{I_{\rho}(f ; y)-f(y)\right\} h(y) d s(y) \quad(0<\rho<\infty)
$$

für jede Funktion $h \in \mathbb{L}^{q}\left(S^{k}\right)(1 / p+1 / q=1)$ eine Menge von stetigen, 
linearen Funktionalen auf $\mathbb{L}^{q}\left(S^{k}\right)$ mit

$$
\left\|F_{\rho}\right\|=[\varphi(\rho)]^{-1}\left\|I_{\rho} f-f\right\|_{p}=O(1) \quad(\rho \rightarrow \infty) .
$$

Daher folgt aus dem Satz über die schwache*-Kompaktheit ([37, S. 37]), daß eine Funktion $g \in \mathbb{L}^{p}\left(S^{k}\right)$ und eine Teilfolge $\rho_{j}$ mit $\lim _{j \rightarrow \infty} \rho_{j}$ $=\infty$ existieren, so daß

$$
\lim _{j \rightarrow \infty} \frac{1}{\Omega_{k}} \int_{S^{k}} \frac{1}{\varphi\left(\rho_{j}\right)}\left\{I_{\rho_{j}}(f ; y)-f(y)\right\} h(y) d s(y)=\frac{1}{\Omega_{k}} \int_{S^{k}} g(y) h(y) d s(y)
$$

für alle Funktionen $h \in \mathbb{L}^{q}\left(S^{k}\right)$ gilt.

Wählt man nun speziell $h(y)=((n+\lambda) / \lambda) P_{n}{ }^{\lambda}[(x, y)](n=0,1,2, \cdots)$ für ein festes $x \in S^{k}$, dann folgt wie im Beweis von Teil a)

$$
\lim _{j \rightarrow \infty} Y_{n}\left\{\frac{1}{\varphi\left(\rho_{j}\right)}\left(I_{\rho_{j}} f-f\right) ; x\right\}=\lim _{j \rightarrow \infty} \frac{\frac{\lambda}{n+\lambda} \hat{K}_{\rho_{j}}(n)-1}{\varphi\left(\rho_{j}\right)} Y_{n}(f ; x)=Y_{n}(g ; x)
$$

und mit (3.2) ist daher

$$
\psi(n) Y_{n}(f ; x)=Y_{n}(g ; x) \quad(n=0,1,2, \cdots) .
$$

Der Satz ist also für die Räume $\boldsymbol{X}=\boldsymbol{C}\left(S^{k}\right)$ und $\boldsymbol{X}=\mathbb{L}^{p}\left(S^{k}\right)(1<p<\infty)$ bewiesen.

$\mathrm{Zu}$ beweisen bleibt noch der Fall $p=1$. Aus einem Satz der Integrationstheorie [48, S. 177] folgt, daß für $0<\rho<\infty$

$$
\nu_{\rho}(E)=[\varphi(\rho)]^{-1} \int_{E}\left[I_{\rho}(f ; y)-f(y)\right] d s(y)
$$

für jede Borelmenge $E$ auf $S^{k}$ eine Familie von Maßen bildet, die absolut stetig bezüglich des Maßes auf $S^{k}$ sind. Weiter gilt (siehe z.B. $[29$, S. 114 , S. 180])

$$
\left\|\nu_{\rho}\right\|_{\text {.r }}=[\varphi(\rho)]^{-1}\left\|I_{\rho} f-f\right\|_{1}=O(1) \quad(\rho \rightarrow \infty),
$$

wobei die Beschränktheit aus der Voraussetzung dieses Satzes folgt. Nun bildet das Integral

$$
F_{\rho}(h)=\frac{1}{\Omega_{k}} \int_{S^{k}} h(y) d \nu_{\rho}(y)
$$

für jedes $h \in \boldsymbol{C}\left(S^{k}\right)$ und jedes $\rho>0$ ein stetiges lineares Funktional auf dem Raum $\boldsymbol{C}\left(S^{k}\right)$ nach dem Darstellungssatz von F. Riesz. 
Die Norm des Funktionals ist gegeben durch $\left\|F_{\rho}\right\|=\left\|\nu_{\rho}\right\|_{M}$. Also bilden nach (3.8) die $F_{\rho}(h)$ eine Menge von stetigen linearen Funktionalen, die gleichgradig bezüglich $\rho(0<\rho<\infty)$ beschränkt sind. Daraus folgt nach dem Satz über die schwache* Kompaktheit auf dem Raum $M\left(S^{k}\right)$ (bekannt als Satz von Helly-Bray), daß eine Teilfolge $\rho_{j}$ (wie oben definiert) und ein (endliches) $\mathrm{Maß} \mu \in \boldsymbol{M}\left(S^{k}\right)$ existiert, so daß für alle Funktionen $h \in \boldsymbol{C}\left(S^{k}\right)$ gilt

$$
\lim _{j \rightarrow \infty} \frac{1}{\Omega_{k}} \int_{S^{k}} h(y) d \nu_{\rho_{j}}(y)=\frac{1}{\Omega_{k}} \int_{S^{k}} h(y) d \mu(y) .
$$

Da $\nu_{\rho_{j}}$ absolut stetig bezüglich ds ist, folgt hier

$$
\frac{1}{\Omega_{k}} \int_{S^{k}} h(y) d \nu_{\rho_{j}}(y)=\frac{1}{\Omega_{k}} \int_{S^{k}} h(y) \frac{I_{\rho_{j}}(f ; y)-f(y)}{\varphi\left(\rho_{j}\right)} d s(y) .
$$

Wählt man nun $h(y)=((n+\lambda) / \lambda) P_{n}^{\lambda}[(x, y)]\left(n=0,1,2, \cdots ; x \in S^{k}\right)$, dann folgt wieder wie im ersten Teil dieses Beweises mit (3.2) die Relation

$$
\psi(n) Y_{n}(f ; x)=Y_{n}\left(d_{\mu} ; x\right) \quad(n=0,1,2, \cdots),
$$

Damit ist der Satz vollständig bewiesen. Die Methode wurde schon in [68] benutzt.

Dieser Satz bestimmt nun eine Klasse $\mathcal{K}$, die der Aussage b) der Definition 3.1 genügt. Um den Saturationssatz für die Operatoren $\left\{I_{\rho} ; 0<\rho<\infty\right\}$ zu beweisen, bleibt noch zu zeigen, daß diese Klasse $\mathcal{K}$ auch die Bedingung c) der Definition erfüllt. Diese Problem ist weitaus schwieriger als das erste. Stellt man nun zusätzlich die Bedingung, daß für $n=1,2, \cdots, 0<\rho<\infty$, gilt

$$
d(\rho, n) \equiv \frac{\frac{\lambda}{n+\lambda} \hat{K}_{\rho}(n)-1}{\varphi(\rho) \psi(n)}=\frac{\lambda}{n+\lambda} \hat{\eta}_{\rho}(n) \quad \text { und } \quad d(\rho, 0)=1,
$$

wobei $\left\{\eta_{\rho}\right\}$ eine Menge von zonalen Funktionen ist, die der Bedingung : $\mid \eta_{\rho} \|_{1, \lambda} \leq M, 0<\rho<\infty$, genügen, dann erhält man den Saturationssatz.

Bedingungen in dieser Form wurden zuerst von $H$. Buchwalter [12] und P. L. Butzer [14, S. 400], dann auch von G. Sunouchi [69, S. 129] gestellt. 
Satz 3.2. Ist $f \in \mathbb{X}$ und das singuläre Integral $I_{\rho} f$ vom Typ (1.23) mit dem Kern $K_{\rho}$, der die Bedingungen (1.20), (1.21), (3.2) und (3.9) mit $\left\|\eta_{\rho}\right\|_{1, \lambda} \leq M, 0<\rho<\infty$, erfüllt, dann folgt für alle $f \in \mathcal{H}(\boldsymbol{X}, \psi(n))$ die Aussage

$$
\left\|I_{\rho} f-f\right\|=O(\varphi(\rho)) \quad(\rho \rightarrow \infty) .
$$

Beweis : Ist z.B. $\boldsymbol{X}=\boldsymbol{L}^{1}\left(S^{k}\right)$, dann ergibt sich aus der Voraussetzung $f \in \mathcal{H}\left(\boldsymbol{L}^{1}\left(S^{k}\right), \psi(n)\right)$ mit Hilfe des Faltungssatzes 1.4 und der Bedingung (3.9)

$$
[\varphi(\rho)]^{-1}\left\|I_{\rho} f-f\right\|_{1}=\left\|\eta_{\rho} * d \mu\right\|_{1} \leq M\|\mu\|_{1:}=O(1) \quad(\rho \rightarrow \infty)
$$

und damit die Behauptung. In den anderen Räumen verläuft der Beweis analog.

Die Klasse $\mathcal{K}$ erfüllt also die Bedingung c) der Definition 3.1 und ist damit die Saturationsklasse des Verfahrens $I_{\rho}$.

Bemerkung 3. 1. Um diese Methode auzuwenden, muß man hinreichende Bedingungen kennen, damit eine Zahlenfolge $\{\hat{\eta}(n)$; $n=0,1,2, \cdots\}$ eine Folge von Gegenbauer-Koeffizienten einer Funktion $\eta \in \mathbb{L}_{\lambda}{ }^{1}$ ist. Solche Bedingungen findet man in [4, I, S. 198]; sie sind allerdings recht kompliziert.

Außerdem hängt in dem hier untersuchten Full die Folge $\{\hat{\eta}(n)\}$ von einem Parameter $\rho$ ab, und wenn also für jedes $\rho$ gilt $\eta_{\rho} \in \boldsymbol{L}_{\lambda}{ }^{1}$, also $\left\|\eta_{\rho}\right\|_{1, \lambda} \leq M_{\rho}$, dann muß die Konstante $M_{\rho}$ noch zusätzlich unabhängig von $\rho$ sein. Für $S^{2}$ werden hinreichende Bedingungen dafür u.a. in [6] angegeben.

Bemerkung 3.2. Der direkte Satz ist im Raum $\boldsymbol{L}^{2}\left(S^{k}\right)$ einfach zu lösen, da jede beschränkte Zahlenfolge einen Multiplikator in $\mathbb{L}^{2}\left(S^{k}\right)$ bildet. (Multiplikatoren werden ausführlicher in Abschnitt 5.3 untersucht). Setzt man voraus, daß für die Folge $\{d(\rho, n)\}$, in (3.9) definiert, gilt

$$
\lceil d(\rho, n) \mid \leq M \quad(n=0,1,2, \cdots ; 0<\rho<\infty),
$$

wobei die Konstante $M$ unabhängig von $n$ und $\rho$ ist, dann folgt aus dem Satz von Riesz-Fischer, daß die Glieder $d(\rho, n) Y_{n}(g ; x), n=0$, 
$1, \cdots$, die Glieder der Laplace-Reihe einer Funktion $l_{\rho} \in \boldsymbol{L}^{2}\left(S^{k}\right), 0<\rho$ $<\infty$, sind, falls $f \in \mathcal{H}\left(\boldsymbol{L}^{2}\left(S^{k}\right), \psi(n)\right)$ ist, also $d(\rho, n) Y_{n}(g ; x)=Y_{n}\left(l_{\rho} ; x\right)$, $n=0,1,2, \cdots$. Die Parsevalsche Gleichung ergibt mit (3.11) und der Beziehung $\psi(n) Y_{n}(f ; x)=Y_{n}(g ; x)$

$$
[\varphi(\rho)]^{-1}\left\|I_{\mathrm{p}} f-f\right\|_{2}=\left\|l_{\mathrm{p}}\right\|_{2} \leq M\|g\|_{2} \quad(\rho \rightarrow \infty) .
$$

Die Bedingung (3.10) ist in allen bekannten Beispielen erfüllt. Es ist hier leicht einzusehen, daß (3.10) nicht nur hinreichend, sondern auch notwendig dafür ist, daß (3.11) in der $\boldsymbol{L}^{2}$-Norm gilt.

Wie schon gesagt, sind in allen Räumen außer $\boldsymbol{L}^{2}\left(S^{k}\right)$ keine einfachen Bedingungen an die Folge $\{d(\rho, n)\}$ für die Lösbarkeit dieses Problems bekannt. Im nächsten Kapitel, wo Satz 3.1 auf spezielle Beispiele angewandt wird, erhält man auch die Umkehrung zu Satz 3.1, b) für jedes hier untersuchte singuläre Integral.

\section{Spezielle Singuläre Integrale}

\section{1. Das singuläre Integral von Weierstraß.}

Als erstes Beispiel wird das singuläre Integral von Weierstra $\beta$ (oder von Gauß oder von Gauß-Weierstraß) untersucht. Es ist definiert durch

$$
W_{t}(f ; x) \equiv \frac{1}{\Omega_{k}} \int_{S^{k}} w_{t}[(x, y)] f(y) d s(y)
$$

für alle $f \in \boldsymbol{X}$, wobei $t$ ein reeller, positiver Parameter ist, und die Funktion $w_{t}(\cos \theta)$ für $t>0$ durch die absolut und gleichmäßig auf $S^{k}$ konvergente Reihe

$$
w_{t}(\cos \theta)=\sum_{n=0}^{\infty} e^{-n(n+2 \lambda) t} \frac{n+\lambda}{\lambda} P_{n}{ }^{\lambda}(\cos \theta) \quad(0 \leq \theta \leq \pi)
$$

gegeben ist. Der Kern $w_{t}(\cos \theta), t>0$, hat die folgenden Eigenschaften :

$$
\begin{array}{lc}
w_{t}(\cos \theta) \geq 0 & (0 \leq \theta \leq \pi), \\
\frac{1}{\Omega_{k}} \int_{S^{k}} w_{t}[(x, y)] d s(y)=1 & \left(x \in S^{k}\right),
\end{array}
$$




$$
\begin{array}{r}
\frac{1}{\Omega_{k}} \int_{S^{k}} w_{t_{1}}[(x, y)] w_{t_{2}}[(y, z)] d s(y)=w_{t_{1}+t_{2}}[(x, z)] \\
\quad\left(x, z \in S^{k} ; t_{1}, t_{2}>0\right) .
\end{array}
$$

Diese drei Eigenschaften wurden von S. Bochner in [9, S. 36] bewiesen. Wichtig ist die Positivität des Kerns. Wegen der absoluten und gleichmäßigen Konvergenz der Reihe (4.1.2) kann man für $W_{t}(f ; x)$ auch schreiben

$$
W_{t}(f ; x)=\sum_{n=0}^{\infty} e^{-n(n+2 \lambda) t} Y_{n}(f ; x) 。
$$

Außerdem hat man

Lemma 4.1.1. Für alle $f \in \boldsymbol{X}$ gilt

$$
\left\|W_{t} f\right\| \leq\|f\|
$$$$
(t>0) \text {, }
$$

Man erhält (4.1.7) mit dem Faltungssatz und den Eigenschaften (4.1.3) und (4.1.4.). (4.1.8) folgt für $\boldsymbol{X}=\boldsymbol{C}\left(S^{k}\right)$ aus dem Satz 2.1.3 über positive Operatoren mit der Bemerkung (3.1). Da $\|f\|_{\boldsymbol{X}} \leq\|f\|_{\boldsymbol{C}}$ für alle $f \in C\left(S^{k}\right)$ ist, folgt auch (4.1.8) in jeder $X$-Norm für alle $f \in \boldsymbol{C}\left(S^{k}\right)$, und da $\boldsymbol{C}\left(S^{k}\right)$ dicht in $\mathbb{L}^{p}\left(S^{k}\right), 1 \leq p<\infty$, ist ([29, S. 170]), gilt mit (4.1.7) und dem Satz von Banach-Steinhaus auch (4.1.8) für alle $f \in \mathbb{L}^{p}\left(S^{k}\right)(1 \leq p<\infty)$.

Die Funktion $W_{t}(f ; x)$ ist außerdem für $t>0$ und jedes $f \in \boldsymbol{X}$ beliebig oft differenzierbar auf $S^{k}$, und sie ist Lösung der Wärmeleitungsgleichung

$$
\frac{\partial}{\partial t} U(x, t)=\Delta U(x, t)
$$

$$
\left(t>0, x \in S^{k}\right)
$$

die der Anfangsbedingung

$$
\lim _{t \rightarrow 0 \vdash} U(x, t)=f(x)
$$

genuigt.

Die Menge von Operatoren $\left\{W_{t}, 0<t<\infty\right\}$, die durch das singuläre Integral von Weierstraß gebildet wird, hat noch eine wesentliche Eigenschaft. Sie bildet eine Halbgruppe von Kontraktionsoperatoren der Klasse $\left(C_{0}\right)$ auf $\boldsymbol{X}$. 
Eine Menge von Operatoren $\left\{T_{t}, 0 \leq t<\infty\right\}$ auf $X$ besitzt diese Eigenschaft, wenn sie die folgenden Bedingungen erfüllt:

$T_{t}$ ist ein beschränkter, linearer Operator von $\boldsymbol{X}$ in sich für jedes $t \in(0, \infty)$ und $T_{0}=I$ (Identität);

Unter diesen Voraussetzungen ist $T_{t} f$ für jedes $f \in \boldsymbol{X}$ eine stark stetige, vektorwertige Funktion mit Definitionsbereich $0 \leq t<\infty$ und Wertebereich in $\boldsymbol{X}$.

Hier sollen noch einige Eigenschaften über die Integration vektorwertiger Funktionen auf einem Intervall $[a, b]$ der reellen Achse angegeben werden. Eine vektorwertige Funktion $h(t)$ von $[a, b]$ in $\boldsymbol{X}$ heißt Bochner-integrierbar, falls sie dort stark messbar ist und das Integral $\int_{a}^{b}\|h(t)\| d t$ im Sinne von Lebesgue existiert. Jede stark stetige Funktion $h(t)$ ist stark messbar. Diese Definitionen und Eigenschaften sind z.B. in [37, S. 71-80] zu finden.

Die Menge von Operatoren $\left\{W_{t} ; 0 \leq t<\infty\right\}$, gegeben durch $W_{t} f$ für jedes $f \in X$, bildet nun wegen (4.1.5), (4.1.7) und (4.1.8) eine Halbgruppe von Kontraktionsoperatoren der Klasse $\left(C_{0}\right)$. Außerdem ist die vektorwertige Funktion $W_{t} f$ für jedes $f$ stark stetig auf $0 \leq t<\infty$, also auch Bochner-integrierbar auf jedem endlichen Intervall.

Der folgende Satz hängt eng mit dem Teil a) von Satz 3.1 zusammen. (Für die Beweismethode vgl. [52, S. 56] und [53, II, S. 56] für Funktionen im $E^{k}$; siehe auch [17] für $E^{1}$ ).

Satz 4.1.2. Für zwei Funktionen $f, g \in \boldsymbol{X}$ sind die folgenden Aussagen äquivalent ;

$$
\begin{aligned}
& -n(n+2 \lambda) Y_{n}(f ; x)=Y_{n}(g ; x) \quad(n=0,1,2, \cdots) \\
& \lim _{t \rightarrow 0+}\left\|\frac{W_{t} f-f}{t}-g\right\|=0 .
\end{aligned}
$$

Ist $g=0$ in $\boldsymbol{X}$, dann gilt $f=$ const. 
Bemerkung. Ist speziell $f$ zweimal stetig differenzierbar auf $S^{k}$ ([65, S. 213]), dann ist $-n(n+2 \lambda) Y_{n}(f ; x)=Y_{n}(\Delta f ; x)$ und (4.1.16) hat die Gestalt

$$
\lim _{t \rightarrow 0+}\left\|\frac{W_{t} f-f}{t}-\Delta f\right\|=0
$$

Beweis des Satzes: Als erstes soll gezeigt werden, daß (4.1.16) aus (4.1.15) folgt. Dazu bildet man das Integral

$$
\int_{0}^{t} W_{\tau} g d \tau
$$

welches wie oben angegeben im Sinne von Bochner für jedes $t>0$ und alle $g \in \boldsymbol{X}$ existiert. Die Kugelfunktion $Y_{n}(h ; x)(h \in \mathbb{X})$ ist für jedes $n=0,1, \cdots$ und jedes feste $x \in S^{k}$ ein lineares, beschränktes Funktional auf dem Raum $\boldsymbol{X}$. Die Bildung des Funktionals kann nach [37, S. 83] mit der Integration vertauscht werden. Mit (4.1.6) gilt daher

$$
\begin{aligned}
Y_{n} & \left\{\int_{0}^{t} W_{\tau} g d \tau ; x\right\}=\int_{0}^{t} Y_{n}\left\{W_{\tau} g ; x\right\} d \tau \\
& =\int_{0}^{t} e^{-n(n+2 \lambda) \tau} Y_{n}(g ; x) d \tau \\
& =\left(e^{-n_{(n+2 \lambda) t}}-1\right) Y_{n}(f ; x) .
\end{aligned}
$$

Die letzte Zeile gilt nach der Voraussetzung (4.1.15) für alle $n=1$, $2, \cdots$ (und auch für $n=0$ ).

Andererseits ist

$$
Y_{n}\left\{W_{t} f-f ; x\right\}=\left(e^{-n(n-2 \lambda) t}-1\right) Y_{n}(f ; x) \quad(n=0,1,2, \cdots) .
$$

Aus diesen beiden Gleichungen folgt mit dem Eindeutigkeitssatz für Laplace-Entwicklungen für alle $x \in S^{k}$ im Raume $\boldsymbol{X}=\boldsymbol{C}\left(S^{k}\right)$ und für fast alle $x \in S^{k}$ in den Räumen $\boldsymbol{X}=\boldsymbol{L}^{p}\left(S^{k}\right)(1 \leq p<\infty)$ die Beziehung

$$
\frac{W_{t}(f ; x)-f(x)}{t}=\frac{1}{t} \int_{0}^{t} W_{\tau}(g ; x) d \tau \text {. }
$$

(Für die Interpretation des Integrals siehe [37, S. 69]). Man erhält mit den Eigenschaften des Bochner-Integrals und mit (4.1.8) 


$$
\left\|\frac{W_{t} f-f}{t}-g\right\|=\left\|\frac{1}{t} \int_{0}^{t}\left[W_{\tau} g-g\right] d \tau\right\| \leq \sup _{0 \leq \tau \leq t}\left\|W_{\tau} g-g\right\| \rightarrow \underset{(t \rightarrow 0+) .}{0}
$$

Damit ist (4.1.16) gültig. Diese Beweismethode wurde in [52, S. 56/57] entwickelt.

Setzt man umgekehrt (4.1.16) voraus, dann kann man Teil a) von Satz 3.1 anwenden. Ersetzt man in $w_{t} f$ den Parameter $t(t \rightarrow 0+)$ durch $1 / \rho(\rho \rightarrow \infty)$, dann erfüllt $W_{1 / \rho} f$ und der Kern $w_{1 / \rho}$ die Voraussetzungen von Satz 3.1 wegen (4.1.2), (4.1.3) und (4.1.4). Es ist noch $\mathrm{zu}$ zeigen, daß (3.2) erfüllt ist. Wegen (4.1.2) ist $\hat{w}_{t}(n)=((n+\lambda) / \lambda) \exp \{-n(n+2 \lambda) t\}, n=0,1,2, \cdots$,

$$
\lim _{t \rightarrow 0+} \frac{e^{-n_{\left(n_{+} 2 \lambda\right) t}}-1}{t}=-n(n+2 \lambda) \quad(n=0,1, \cdots)
$$

mit $\psi(n)=-n(n+2 \lambda)$. Nach Satz 3.1 Teil a) folgt daher aus (4.1.16) die Aussage (4.1.15), womit die Äquivalenz von (4.1.15) und (4.1.16) gezeigt ist.

Ist nun $g=0$ in $\boldsymbol{X}$, dann ist $Y_{n}(g ; x)=0$ für alle $n=0,1, \cdots$ und wegen (4.1.15) auch $Y_{n}(f ; x)=0$ für alle $n$ bis auf $n=0$. Wir haben also $Y_{n}\left\{f-Y_{0} f ; x\right\}=0$ für alle $n=0,1, \cdots$ und daher $f(x)=Y_{0}(f ; x)$ $=$ const. in $\boldsymbol{X}$. Der Satz ist jetzt vollständig bewiesen.

Wir kommen nun zur Anwendung von Satz 3.1, Teil b).

Satz 4.1.3. Für ein $f \in \boldsymbol{X}$ sind die folgenden Aussagen äquivalent :

$$
\begin{array}{ll}
\left\|W_{t} f-f\right\|=O(t) & (t \rightarrow 0+) ; \\
f \in \mathcal{H}(\boldsymbol{X},-n(n+2 \lambda)) . &
\end{array}
$$

Beweis: Die Aussage, daß aus (4.1.21) folgt $f \in \mathscr{H}(\boldsymbol{X},-n(n+2 \lambda))$, ergibt sich als Anwendung von Teil b) des Satzes 3.1. Im Beweis zu Satz 4.1.2 wurde schon gezeigt, daß alle Voraussetzungen dazu erfüllt sind. Damit gilt (4.1.22).

Wird umgekehrt (4.1.22) vorausgesetzt, dann ist im Falle $\boldsymbol{X}=$ $\boldsymbol{L}^{p}\left(S^{k}\right)(1<p<\infty)$ leicht auf (4.1.21) zu schließen. Denn aus $f \in$ $\mathscr{H}(\boldsymbol{X},-n(n+2 \lambda))$ d.h. $-n(n+2 \lambda) Y_{n}(f ; x)=Y_{n}(g ; x)$ mit $f, g \in \mathbb{L}^{p}\left(S^{k}\right)$ folgt aus (4.1.19) die Beziehung $W_{t} f-f=\int_{0}^{t} W_{\tau} g d \tau$ und damit 


$$
\left\|W_{t} f-f\right\|_{p} \leq i \mid g \|_{p} t=O(t)
$$

Wir untersuchen nun den Raum $\boldsymbol{X}=\boldsymbol{L}^{1}\left(S^{k}\right)$. Es existiert wegen (4.1.22) ein $\mathrm{Maß} \mu \in \mathbb{M}\left(S^{k}\right)$, so daß gilt $-n(n+2 \lambda) Y_{n}(f ; x)=Y_{n}\left(d_{\mu} ; x\right)$. Die Faltung des Kernes $w_{t}$ mit einem Maß $\mu$ ist durch (1.17) in der Form

$$
\left[w_{t} * d \mu\right](x)=\frac{1}{\Omega_{k}} \int_{S^{k}} w_{t}[(x, y)] d \mu(y)
$$

gegeben. Der Faltungssatz 1.4 besagt, daß $w_{t} * d_{\mu} \in L^{1}\left(S^{k}\right)$ für jedes $t>0$ ist und

$$
\left\|w_{t} * d_{\mu}\right\|_{1} \leq\left\|w_{t}\right\|_{1, \lambda}\|\mu\|_{M} \leq\|\mu\|_{M}
$$

Wegen (4.1.3) und (4.1.4). Wir wollen zeigen, daß auch hier eine ähnliche Formel wie (4.1.19) gilt. Als erstes wird behauptet, daß die vektorwertige Funktion $h(t)=w t * d \mu$ definiert auf $0<t<\infty$ mit Wertebereich in $\mathbb{L}^{1}\left(S^{k}\right)$ für jedes $\mu \in \boldsymbol{M}\left(S^{k}\right)$ stark stetig auf dem Intervall $[\varepsilon, \infty)(\varepsilon>0)$ ist. Es gilt für ein $t>\varepsilon h(t)=w_{t-\varepsilon} * w_{t} * d_{\mu}=$ $w_{t-\varepsilon} * h(\varepsilon)$. Damit ist für zwei Zahlen $t_{1}, t_{2}$ mit $0<\varepsilon \leq t_{2}<t_{1}$

$$
\begin{aligned}
\left\|h\left(t_{1}\right)-h\left(t_{2}\right)\right\|_{1} & =\left\|w_{t_{1}-} * h(\varepsilon)-w_{t_{2}-\varepsilon} * h(\varepsilon)\right\|_{1} \\
& \leq\left\|W_{t_{2}-\varepsilon}\right\|\left\|W_{t_{1}-t_{2}} h(\varepsilon)-h(\varepsilon)\right\|_{1} \leq\left\|W_{t_{1}-t_{2}} h(\varepsilon)-h(\varepsilon)\right\|_{1},
\end{aligned}
$$

wobei wir die Schreibweise $w_{t-\varepsilon} * h(\varepsilon)=W_{t-\varepsilon} h(\varepsilon)$ und $\left\|W_{t-\varepsilon}\right\| \leq 1$ wie in (4.1.7) benutzt haben. Der letzte Ausdruck konvergiert wegen (4.1.8) gegen Null für $t_{1} \rightarrow t_{2}$. Also ist die Funktion $h(t)$ stark stetig auf $[\varepsilon, \infty)$ für jedes $\varepsilon>0$. Weiter gilt

$$
\int_{\varepsilon}^{t}\|h(\tau)\|_{1} d \tau \leq \int_{\varepsilon}^{t}\|\mu\|_{M} d \tau<\|\left.\mu\right|_{\text {MM }} t
$$

wobei die rechte Seite unabhängig von $\varepsilon$ ist. Damit existiert das Integral $\int_{0}^{t} h(\tau) d \tau=\int_{0}^{t}\left[w_{\tau} * d \mu\right] d \tau$ im Sinne von Bochner für jedes $\mu \in \boldsymbol{M}\left(S^{k}\right)$, und es gilt wieder wie in (4.1.17) mit $-n(n+2 \lambda) Y_{n}(f ; x)$ $=Y_{n}\left(d_{\mu} ; x\right)$ die Formel

$$
Y_{n}\left\{\int_{0}^{t}\left[w_{\tau} * d \mu\right] d \tau ; x\right\}=\left(e^{-n(n+2 \lambda) t}-1\right) Y_{n}(f ; x)
$$

und mit (4.1.18) folgt $W_{t} f-f=\int_{0}^{t}\left[w_{t} * d \mu\right] d \tau$, und damit gilt die 
Behauptung. Der Satz ist also für $\boldsymbol{X}=\boldsymbol{L}^{1}\left(S^{k}\right)$ bewiesen.

Es bleibt noch der Fall $\boldsymbol{X}=\boldsymbol{C}\left(S^{k}\right)$ zu behandeln. Entsprechend dem Raum $\boldsymbol{L}^{1}\left(S^{k}\right)$ läßt sich hier zeigen

$$
\left\|W_{t} f-f\right\|_{C} \leq\|g\|_{\infty} \cdot t \quad\left(f \in \mathcal{H}\left(C\left(S^{k}\right),-n(n+2 \lambda)\right),\right.
$$

womit der Satz vollständig bewiesen ist.

Folgerung 4.1.4. Das singuläre Integral von Weierstra $\beta$ ist in den Räumen $\boldsymbol{L}^{p}\left(S^{k}\right), 1 \leq p<\infty$, bzw. $\boldsymbol{C}\left(S^{k}\right)$ saturiert mit der Ordnung $O(t)(t \rightarrow 0+)$ und die Saturationsklasse ist gegeben durch die Menge $\mathcal{H}\left(\boldsymbol{L}^{p}\left(S^{k}\right),-n(n+2 \lambda)\right)$ bzw. $\mathcal{H}\left(\boldsymbol{C}\left(S^{k}\right),-n(n+2 \lambda)\right)$.

Wie wir in der Bemerkung zu Satz 4.1.2 gesehen haben, sind in der Saturationsklasse alle Funktionen $f$ enthalten, die zweimal stetig differenzierbar sind. Sicher sind mehr Funktionen darin enthalten. Im Raume $\boldsymbol{C}\left(S^{k}\right)$ z.B. besitzt die Funktion $g \in \boldsymbol{L}^{\infty}\left(S^{k}\right)$ dieselbe LaplaceEntwicklung wie $\Delta f$, wenn die Ableitungen existieren. Die Funktion $g$ ist nur wesentlich beschränkt, also braucht $\Delta f$ nur fast überall zu existieren und wesentlich beschränkt zu sein, jedoch nicht stetig. Dies ist im Falle $S^{2}$ (bei eindimensionalen Fourierreihen) gültig. Dort folgt aus (4.1.22), daß $f$ einmal stetig differenzierbar, die zweite Ableitung wesentlich beschränkt und $f^{\prime \prime}=g$ ist. Siehe [25] oder [19, Ch. II].

Als nächstes werden die Funktionen der Klassen $\mathcal{H}(\boldsymbol{X},-n(n+$ $2 \lambda)$ ) als Faltungsintegrale mit einem Kern $k$ dargestellt.

Lemma 4.1.5. Für ein $f \in \boldsymbol{X}$ sind die folgenden Behauptungen äquivalent :

a) $f \in \mathcal{H}(\boldsymbol{X},-n(n+2 \lambda))$,

b) Es gilt fast überall für $f \in \mathbb{L}^{p}\left(S^{k}\right)$ mit $g \in \mathbb{L}^{p}\left(S^{k}\right)(1<p<\infty)$, überall im Falle $f \in \boldsymbol{C}\left(S^{k}\right)$ mit $g \in \boldsymbol{L}^{\infty}\left(S^{k}\right)$ die Darstellung

$$
f(x)=Y_{0}(f ; x)-\frac{1}{\Omega_{k}} \int_{S^{k}} k(1,(x, y)) g(y) d s(y)
$$

oder fast überall für $f \in \boldsymbol{L}^{1}\left(S^{k}\right)$ mit einem $M a \beta \mu \in M\left(S^{k}\right)$

$$
f(x)=Y_{0}(f ; x)-\frac{1}{\Omega_{k}} \int_{S^{k}} k(1,(x, y)) d \mu(y) .
$$


Hierbei ist $k(1, t),-1 \leq t<1$, gegeben durch

$$
k(1, t)=\frac{1}{2 \lambda} \int_{0}^{1} \frac{1-r^{2}}{r}\left[\frac{1-r^{2}}{\left(1-2 r t+r^{2}\right)^{\lambda+1}}-1\right] d r
$$

mit $\| k\left(1, \cos \theta \|_{q, \lambda}<\infty\right.$ fïr $1 \leq q<(2 \lambda+1) /(2 \lambda-1)$ im Falle $\lambda>1 / 2$ und $1 \leq q<\infty$, falls $\lambda=1 / 2$.

Beweis: Als erstes sollen die Eigenschaften der Funktion $k$ bewiesen werden. Man benutzt die Formel (1.4) in der Gestalt

$$
\sum_{n=1}^{\infty} r_{1}^{n+\beta} \frac{n+\lambda}{\lambda} P_{n}^{\lambda}(t)=\left[\frac{1-r_{1}^{2}}{\left(1-2 r_{1} t+r_{1}^{2}\right)^{\lambda+1}}-1\right] r_{1} \quad\left(0 \leq r_{1}<1 ; \beta \geq-1\right) .
$$

Man erhält durch Integration bezüglich $r_{1}$ von 0 bis $r<1$, die auf der linken Seite gliedweise wegen der gleichmäßigen Konvergenz der Reihe durchgeführt wird,

$$
\sum_{n=1}^{\infty} \frac{r^{n+\beta \perp 1}}{n+\beta+1} \frac{n+\lambda}{\lambda} P_{n}^{\lambda}(t)=\int_{0}^{r}\left\{\frac{1-r_{1}^{2}}{\left(1-2 r_{1} t+r_{1}^{2}\right)^{\lambda-1}}-1\right\} r_{1}^{\beta} d r_{1} .
$$

Wählt man zuerst $\beta=-1$ und multipliziert die Gleichung mit $r^{2 \lambda}$, dann $\beta=2 r-1$ und subtrahiert beide Ausdrücke voneinander, dann erhält man

$$
\sum_{n=1}^{\infty}\left(\frac{1}{n}-\frac{1}{n+2 \lambda}\right) r^{n-2 \lambda} \frac{n+\lambda}{\lambda} P_{n}^{\lambda}(t)=\int_{0}^{r} \frac{r^{2 \lambda}-r_{1}^{2 \lambda}}{r_{1}}\left\{\frac{1-r_{1}^{2}}{\left(1-2 r_{1} t+r_{1}^{2}\right)^{\lambda+1}}-1\right\} d r_{1} .
$$

Es ist also für $0 \leq r<1$ und $-1 \leq t \leq 1$

$$
\begin{aligned}
k(r, t) & \equiv \frac{1}{2 \lambda} \int_{0}^{r} \frac{r^{2 \lambda}-r_{1}^{2 \lambda}}{r_{1}}\left\{\frac{1-r_{1}^{2}}{\left(1-2 r_{1} t+r_{1}^{2}\right)^{\lambda+1}}-1\right\} d r_{1} \\
& =\sum_{n=1}^{\infty} \frac{r^{n+2 \lambda}}{n(n+2 \lambda)} \frac{n+\lambda}{\lambda} P_{n}^{\lambda}(t) .
\end{aligned}
$$

Aus dieser Integraldarstellung folgt direkt, da $\left(1-2 r t+r^{2}\right)>0$ für $0 \leq r \leq 1,-1 \leq t<1$, daß der Grenzwert $\lim _{r \rightarrow 1-} k(r, t)$ für jedes $t$ mit $-1 \leq t<1$ existiert und die dadurch definierte Funktion $k(1, t)$ ebenfalls für $-1 \leq t<1$ stetig ist. Um zu zeigen, daß $k(1, t)$ bezüglich $t$ auf $(0,1)$ integrierbar ist, gehen wir genau wie in $[49,5.81]$ vor, wo eine sehr ähnliche Funktion untersucht wurde. An Stelle der Folge $n(n+2 \lambda)$ untersucht man dort die Folge $n^{\alpha}, \alpha>0$. 
Wir haben hier also die Darstellung (4.1.27). Spaltet man das Integral in (4.1.27) in zwei Teile auf, von 0 bis $1 / 2$ und von $1 / 2$ bis 1, dann sieht man aus der Summendarstellung (1.4), daß das erste Integral gleichmäßig in $t$ beschränkt ist. Im zweiten kann das konstante Glied $(1 / 2 \lambda) \int_{1 / 2}^{1}\left[(1-r)^{2 \lambda} / r\right] d r$ weggelassen werden. Es bleibt das Integral

$$
I(t)=\frac{1}{2 \lambda} \int_{1 / 2}^{1} \frac{\left(1-r^{2 \lambda}\right)\left(1-r^{2}\right)}{\left(1-2 r t+r^{2}\right)^{\lambda+1}} \frac{d r}{r}
$$

zu untersuchen. Für $-1 \leq t \leq 3 / 4$ ist $I(t)$ gleichmäßig beschränkt. Für $3 / 4 \leq t<1$ spaltet man des Integral $I(t)$ auf in ein Integral, wo $(1-r)^{2} \geq 1-t$, und ein anderes, wo $(1-r)^{2}<1-t$ ist. Aus der Beziehung $\left(1-2 r t+r^{2}\right)=(1-r)^{2}+2 r(1-t)$ folgen die Ungleichungen (s. [49, S. 81])

$$
\left(1-2 r t+r^{2}\right)^{\lambda+1} \geq(1-r)^{2 \lambda+2}
$$

und

$$
\left(1-2 r t+r^{2}\right)^{\lambda+1} \geq(1-t)^{\lambda+1} \quad(r \geq 1 / 2) .
$$

Man erhält mit $\left(1-r^{2 \lambda}\right)=(1-r)\left(1+r+r^{2}+\cdots+r^{2 \lambda-1}\right) \leq 2 \lambda(1-r)$

$$
\begin{aligned}
|I(t)| & \leq 4 \int_{1 / 2}^{1-(1-t) 1 / 2}(1-r)^{-2 \lambda} d r+2(1-t)^{-\lambda-1} \int_{1-(1-t)^{1 / 2}}^{1}(1-r)^{2} d r \\
& \leq \begin{cases}C_{1}(\lambda)+C_{2}(\lambda)(1-t)^{-\lambda+(1 / 2)} & (\lambda>1 / 2), \\
C_{1}+C_{2}|\log (1-t)| & (\lambda=1 / 2) .\end{cases}
\end{aligned}
$$

Daraus folgt also, daß das ganze Integral $k(1, \cos \theta), t=\cos \theta$, beschränkt ist in $0<\varepsilon \leq \theta \leq \pi$ und für $\theta \rightarrow 0+$ gilt

$$
|k(1, \cos \theta)|= \begin{cases}O(\sin \theta / 2)^{-2 \lambda+1} & (\lambda>1 / 2), \\ O|\log (\sin \theta / 2)| & (\lambda=1 / 2) .\end{cases}
$$

Es ist also

$$
\|k(1, \cos \theta)\|_{q, \lambda}^{q}=\frac{\Omega_{k-1}}{\Omega_{k}} \int_{0}^{\pi}|k(1, \cos \theta)|^{q}(\sin \theta)^{2 \lambda} d \theta<\infty
$$

für alle $q$ mit $1 \leq q<(2 \lambda+1) /(2 \lambda-1)(\lambda>1 / 2)$ und $1 \leq q<\infty$ für $\lambda=1 / 2$.

Damit sind die Eigenschaften der Funktion $k$ aus Lemma 4.1.5 
bewiesen. (Ersetzt man $n(n+2 \lambda)$ durch $n^{2}$, so stimmen diese Ergebnisse mit denen in [49, S. 81] überein. Noch allgemeinere Koeffiizienten als $n^{\alpha}, \alpha>0$, wurden in $[4, \mathrm{I}]$ untersucht).

Wir kommen nun zum Beweis der Darstellungen (4.1.25) und (4.1.26). Nach (4.1.28) gilt, daß die Gegenbauer-Koeffizienten der Funktion $k(1, \cos \theta)$ durch

$$
\hat{k}(1, n)=\frac{n+\lambda}{\lambda} \frac{1}{n(n+2 \lambda)} \quad(n=1,2, \cdots), \hat{k}(1,0)=0
$$

gegeben sind. Also ist, wenn wir $f \in \mathcal{H}(\boldsymbol{X},-n(n+2 \lambda))$ voraussetzen,

$$
Y_{n}(f ; x)=-\frac{\lambda}{n+\lambda} \hat{k}(1, n) Y_{n}(g ; x) \begin{array}{r}
\left(n=1,2, \cdots ; \boldsymbol{X}=\boldsymbol{L}^{p},\right. \\
1<p<\infty, \text { oder } \boldsymbol{X}=\boldsymbol{C})
\end{array}
$$

oder

$$
\begin{array}{r}
Y_{n}(f ; x)=-\frac{\lambda}{n+\lambda} \hat{k}(1, n) Y_{n}(d \mu ; x) \\
\left(n=1,2, \cdots ; \boldsymbol{X}=\boldsymbol{L}^{1}\left(S^{k}\right)\right) .
\end{array}
$$

Daraus folgt mit dem Faltungssatz 1.3 für $\boldsymbol{X}=\mathbb{L}^{p}, 1<p<\infty$, oder $\boldsymbol{X}=\boldsymbol{C}$

$$
f(x)=Y_{0}(f ; x)-\frac{1}{\Omega_{k}} \int_{S^{k}} k(1,(x, y)) g(y) d s(y)
$$

fast überall, und die rechte Seite existiert überall für die Werte von $p$, für die $k \in \boldsymbol{L}_{\lambda}{ }^{q}, 1 / p+1 / q=1$. Für $q$ gilt $1 \leq q<(2 \lambda+1) /(2 \lambda-1)$ nach (4.1.27) und damit existiert die rechte Seite von (4.1.25) überall für $f \in \mathbb{L}^{p}\left(S^{k}\right)$ mit $p>(2 \lambda+1) / 2=(k-1) / 2$.

Ist nun $f \in L^{1}\left(S^{k}\right)$ und gilt a), dann folgt wie oben die Darstellung (4.1.26) fast überall. Aus a) folgt also b). Der Schluß von a) nach b) ist genau eine Aussage der Faltungssätze 1.3 und 1.4, womit Lemma 4.1 .5 bewiesen ist.

Bemerkung. Im Falle $S^{3}$ besitzt (4.1.25) die Gestalt (s. [61, S. 293])

$$
f(x)=Y_{0}(f ; x)+\frac{1}{4 \pi} \int_{s^{3}} \log [1-(x, y)] g(y) d s(y) .
$$

Für Funktionen auf $S^{2}$ erhält man an Stelle von (4.1.25) 


$$
\begin{aligned}
& f(\phi)=\frac{1}{2 \pi} \int_{-\pi}^{\pi} f\left(\phi^{\prime}\right) d \phi^{\prime}-\frac{1}{2 \pi} \int_{-\pi}^{\pi} k\left(1, \cos \left(\phi-\phi^{\prime}\right)\right) g\left(\phi^{\prime}\right) d \phi^{\prime} \\
& k(1, \cos \phi)=\sum_{n=1}^{\infty} \frac{\cos n \phi}{n^{2}}=\frac{1}{4} \phi^{2}-\frac{\pi}{2}|\phi|+\frac{\pi^{2}}{6} \quad(-\pi \leq \phi<\pi) .
\end{aligned}
$$

\section{2. Mittelbildungen von Funktionen auf der Kugel.}

Ziel dieses Abschnittes ist eine weitere Charakterisierung der Klassen $\mathscr{H}(\boldsymbol{X},-n(n+2 \lambda))$ aus Abschnitt 4.1. Hierzu werden zwei Mittelwerte der Funktion auf $S^{k}$ definiert. Das erste Mittel entspricht auf $S^{2}$ (dem Einheitskreis) dem Integralmittelwert

$$
f_{h}(x)=\frac{1}{2 h} \int_{-h}^{h} f(x+u) d u \quad(-\pi \leq x \leq \pi),
$$

dessen Fourierreihe von Lebesgue zur Summation der Fourierreihe von $f$ benutzt wurde. (s. [74, S. 71, S. 321]). Die Fourierreihe des zweiten Integralmittelwertes bildet das Summationsverfahren von Riemann (s. [74, S. 319]). Wir definieren hier die Mittel $M_{h}(f ; x)$ für jedes $f \in L^{1}\left(S^{k}\right)$ durch

$$
M_{h}(f ; x)=\frac{1}{|D(x, h)|} \int_{D(x, h)} f(y) d s(y) \quad(h>0),
$$

wobei die Kugelkappe $D(x, h)$ mit Mittelpunkt $x$ gegeben ist durch $D(x, h)=\left\{y ; y \in S^{k}\right.$ und $\left.(x, y) \geq \cos h\right\} .|D(x, h)|$ ist ihre $(k-1)-$ dimensionale Oberfläche und (siehe z.B. [42, S. 319])

$$
|D(x, h)|=\Omega_{k-1} \int_{0}^{h}(\sin \theta)^{2 \lambda} d \theta .
$$

Wir schreiben immer $D(h)$ an Stelle von $|D(x, h)|$, da die Oberfläche unabhängig von $x$ ist. Man kann $M_{h}(f ; x)$ auch als singuläres Integral in der Form (1.23) schreiben, wenn man $\rho=1 / h$ setzt und den $\operatorname{Kern} k_{\rho}[(x, y)]$ durch

$$
k_{\rho}[(x, y)]=\left\{\begin{array}{cl}
\frac{\Omega_{k}}{D(h)}, & y \in D(x, h) \\
0, & \text { sonst }
\end{array}\right.
$$

definiert.

Lemma 4.2.1. Für alle $f \in X$ gilt 


$$
\left\|M_{h} f\right\| \leq\|f\|
$$

$$
\lim _{h \rightarrow 0+} \mid i M_{h} f-f \|=0 \text {. }
$$

Weiter hat man $([64$, S. $518,521,523])$

$$
Y_{n}\left(M_{h} f ; x\right)=B_{n}^{\lambda}(h) Y_{n}(f ; x)
$$$$
(n=0,1,2, \cdots)
$$

mit

$$
B_{n}^{\lambda}(h)=\frac{\Omega_{k-1}}{P_{n}^{\lambda}(1) D(h)} \int_{0}^{h} P_{n}^{\lambda}(\cos \theta)(\sin \theta)^{2 \lambda} d \theta .
$$

Man kann andere Mittel definieren, indem man die Integration nur über den Rand $(x, y)=\cos h$ von $D(x, h)$ ausführt. Diese Mittel sind definiert durch

$$
S_{h}(f ; x)=\frac{1}{\Omega_{k-1}(\sin h)^{2 \lambda}} \int_{(x, y)=\cos h} f(y) d t(y),
$$

wobei $d t$ das $(k-2)$-dimensionale Oberflächenelement der Fläche $(x, y)=\cos h$ ist. Zwischen den Mitteln $M_{h}$ und $S_{h}$ besteht die Beziehung

$$
\begin{aligned}
M_{h}(f ; x) & =\frac{1}{D(h)} \int_{0}^{h} \int_{(x, y)=\cos u} f(y) d t(y) d u \\
& =\frac{\Omega_{k-1}}{D(h)} \int_{0}^{h}(\sin u)^{2 \lambda} S_{u}(f ; x) d u .
\end{aligned}
$$

Wir wollen nun die Laplace-Entwicklung von $S_{h}(f ; x)$ bestimmen. Es gilt mit (4.2.9)

$$
Y_{n}\left(M_{h} f ; x\right)=\frac{\Omega_{k-1}}{D(h)} \int_{0}^{h}(\sin u)^{2 \lambda} Y_{n}\left(S_{u} f ; x\right) d u .
$$

Setzt man auf der linken Seite (4.2.6) ein, dann folgt

$$
Y_{n}(f ; x) \int_{0}^{h} P_{n}^{\lambda}(\cos \theta)(\sin \theta)^{2 \lambda} d \theta=P_{n}^{\lambda}(1) \int_{0}^{h}(\sin u)^{2 \lambda} Y_{n}\left(S_{u} f ; x\right) d u
$$

und durch Differentiation nach $h$

$$
Y_{n}\left(S_{h} f ; x\right)=\frac{P_{n}^{\lambda}(\cos h)}{P_{n}^{\lambda}(1)} Y_{n}(f ; x) \quad(n=0,1, \cdots) .
$$

Für $S_{h} f$ gilt ebenso wie für $M_{h} f$ 
Lemma 4.2.2. Für alle $f$ aus $\boldsymbol{X}$ gilt

$$
\begin{aligned}
& \left\|S_{h} f\right\| \leq\|f\| \quad(h>0), \\
& \lim _{h \rightarrow 0+}\left\|S_{h} f-f\right\|=0 .
\end{aligned}
$$

Bemerkung 4.2.13. Die Mittel $M_{h}$ werden von $V$. L. Shapiro ([64]) bei Untersuchungen über die Summierbarkeit von LaplaceReihen benutzt. In [66] definiert er mit Hilfe von $M_{h}$ einen verallgemeinerten Laplace-Operator ([66, S. 16]) auf $S^{3}$ und eine sogenannte Lipschitzbedingung auf $S^{k}[66, \mathrm{~S} .6]$, die er zum Beweis der absoluten Konvergenz von Laplace-Reihen benutzt. In die Theorie der partiellen Differentialgleichungen hat C. Herglotz (vgl. [50, S. 248] und [57, S. 38]) diese Mittel eingeführt.

Für $k=3$ findet man die Mittel $S_{h}$ bei $W$. Rudin [61], der dadurch einen verallgemeinerten Laplace-Operator auf der Kugel und die Riemann-Summierbarkeit von Laplace-Reihen definiert hat. (Seine Ergebnisse hat A. Siege! [67] erweitert).

In der Approximationstheorie definieren G. G. Kušnirenko ([43], [44]) und A.S. Džafarov [31] durch diese Mittel Funktionenklassen, die Lipschitzklassen in den Sätzen vom Bernsteinschen und Jacksonschen Typ ersetzen (siehe auch D. L. Ragozin [60]). In der Approximationstheorie im $E^{k}$ werden Mittel analog $\mathrm{zu} S_{h}$ in [71, S. 421] untersucht und im Fall der nichtsaturierten Approximation mit Lipschitzbedingungen verglichen. Ebenfalls im $E^{k}$ hat $R$. J. Nessel die den Integralen $S_{h} f$ und $M_{h} f$ entsprechenden Mittel genau untersucht und sie zur Bestimmung der Saturationsklasse einiger singulärer Integrale herangezogen (s. [52, Abschn, 4.3 und 4.4] und [53, Abschn. $6,7,8])$. Entsprechende Sätze werden wir auf der Kugel für die beiden Mittel beweisen. Dazu benötigen wir das folgende Lemma (vgl. [52, S. 71, S. 80]).

Lemma 4.2.3. Besteht zwischen den Funktionen $f$ und $g$ aus $\boldsymbol{X}$ die Beziehung (4.1.15), dann gilt fast überall (und überall für $\boldsymbol{X}=$ $\left.C\left(S^{k}\right)\right)$ 


$$
\begin{aligned}
S_{h}(f ; \cdot)-f(\cdot) & =\frac{1}{\Omega_{k-1}} \int_{0}^{h} M_{\tau}(g ; \cdot) D(\tau)(\sin \tau)^{-2 \lambda} d \tau \\
& \equiv J_{h}(g ; \cdot)
\end{aligned}
$$

und

$$
M_{h}(f ; \cdot)-f(\cdot)=\frac{\Omega_{k-1}}{D(h)} \int_{0}^{h} J_{\tau}(g ; \cdot)(\sin \tau)^{2 \lambda} d \tau .
$$

Beweis: Der Ausdruck für $B_{n}{ }^{\lambda}(h)$ in (4.2.7) kann in anderer Form geschrieben werden. Mit Hilfe der Formel von Rodriguez (s. [32, I, S. 175]) erhält man

$$
\int_{0}^{h} P_{n}^{\lambda}(\cos \theta)(\sin \theta)^{2 \lambda} d \theta=\frac{2 \lambda}{n(n+2 \lambda)}(\sin h)^{2 \lambda+1} P_{n-1}^{\lambda+1}(\cos h)
$$

(siehe auch [50, S. 248]) und damit

$$
\begin{aligned}
& \frac{1}{\Omega_{k-1}} \int_{0}^{h} B_{n}{ }^{\lambda}(\tau) D(\tau)(\sin \tau)^{-2 \lambda} d \tau \\
& \quad=\frac{2 \lambda}{n(n+2 \lambda) P_{n}^{\lambda}(1)} \int_{0}^{h} P_{n-1}^{\lambda+1}(\cos \tau) \sin \tau d \tau .
\end{aligned}
$$

Nach der Substitution $\cos \tau=u$ führt die Formel (siehe [32, II, S. 176]

$$
\frac{d}{d u} P_{n}^{\lambda}(u)=2 \lambda P_{n-1}^{\lambda+1}(u)
$$

$\mathrm{zu}$

$$
\frac{1}{\Omega_{k-1}} \int_{0}^{h} B_{n}^{\lambda}(\tau) D(\tau)(\sin \tau)^{-2 \lambda} d \tau=\frac{1}{n(n+2 \lambda)}\left\{1-\frac{P_{n}^{\lambda}(\cos h)}{P_{n}^{\lambda}(1)}\right\} .
$$

Wir untersuchen nun die rechte Seite von (4.2.14). Genau wie bei der Gleichung (4.1.17) kann man auch hier zeigen, daß das Funktional $Y_{n}$ mit der Integration von 0 bis $h$ vertauscht werden kann. Daher gilt mit (4.2.6) und (4.2.19)

$$
\begin{aligned}
Y_{n}\left\{J_{h} g ; x\right\} & =\frac{Y_{n}(g ; x)}{\Omega_{k-1}} \int_{0}^{h} B_{n}{ }^{\lambda}(\tau) D(\tau)(\sin \tau)^{-2 \lambda} d \tau \\
& =-\frac{1}{n(n+2 \lambda)} Y_{n}(g ; x)\left\{\frac{P_{n}{ }^{\lambda}(\cos h)}{P_{n}{ }^{\lambda}(1)}-1\right\} \\
& =Y_{n}\left\{S_{h} f-f ; x\right\} .
\end{aligned}
$$


Die letzte Umrechnung folgt aus der Voraussetzung (4.1.15) und (4.2.10). Dies gilt auch für $n=0$, da $Y_{0}(g ; x)=Y_{0}\left(S_{h} f-f ; x\right)=0$ auf $S^{k}$ ist. Daraus folgt (4.2.14) mit dem Eindeutigkeitssatz. Zum Beweis von (4.2.15) integriert man (4.2.19) und erhält

$$
\begin{aligned}
& \frac{1}{\Omega_{k-1}} \int_{0}^{\eta}(\sin h)^{2 \lambda} d h \int_{0}^{h} B_{n}{ }^{\lambda}(\tau)(\sin \tau)^{-2 \lambda} D(\tau) d \tau \\
& \quad=\frac{1}{n(n+2 \lambda)}\left\{\int_{0}^{\eta}(\sin h)^{2 \lambda} d h-\frac{1}{P_{n}^{\lambda}(1)} \int_{0}^{\eta} P_{n}^{\lambda}(\cos h)(\sin h)^{2 \lambda} d h\right\} \\
& =\frac{D(\eta)}{\Omega_{k-1} n(n+2 \lambda)}\left(1-B_{n}^{\lambda}(\eta)\right)
\end{aligned}
$$

mit (4.2.7). Dividiert man die letzte Gleichung durch $D(\eta)$, multipliziert mit $Y_{n}(g ; x)$, benutzt (4.2.6) und die Voraussetzung (4.1.15), dann erhält man

$$
\begin{aligned}
& \frac{Y_{n}(g ; x)}{D(\eta)} \int_{0}^{\eta}(\sin h)^{2} d h \int_{0}^{h} B_{n}^{\lambda}(\tau)(\sin \tau)^{-2 \lambda} D(\tau) d \tau \\
& \quad=\left[B_{n}^{\lambda}(\eta)-1\right] Y_{n}(f ; x)=Y_{n}\left\{M_{\eta} f-f ; x\right\} .
\end{aligned}
$$

Auf der linken Seite ist $Y_{n}(g ; x) B_{n}{ }^{\lambda}(\tau)=Y_{n}\left(M_{\tau} g ; x\right)$ und die Integration von 0 bis $\eta$ kann wieder mit dem Funktional $Y_{n}$ vertauscht werden; daher folgt nach dem Eindeutigkeitssatz die Formel (4.2.15).

Die Beziehungen (4.2.14) und (4.2.15) sind grundlegend in den Beweisen der nächsten Sätze. Sie ersetzen hier die Relation (4.1.19) im Beweis von Satz 4.1.2.

Satz 4.2.4. Für zwei Funktionen $f$ und $g$ aus $\boldsymbol{X}$ sind die folgenden Aussagen äquivalent:

$$
\begin{aligned}
& -\frac{n(n+2 \lambda)}{2(2 \lambda+3)} Y_{n}(f ; x)=Y_{n}(g ; x) ; \\
& \left\|\frac{M_{h} f-f}{h^{2}}-g\right\|=o(1) \quad(h \rightarrow 0+) .
\end{aligned}
$$

Beweis : $M_{h}(f ; x)$ erfüllt die Bedingungen, die in Satz 3.1 an das singuläre Integral $I_{\rho}(f ; x)=M_{h}(f ; x)$ mit $\rho=1 / h$ gestellt werden. $\mathrm{Zu}$ berechnen ist

$$
\lim _{h \rightarrow 0+} \frac{B_{n}^{\lambda}(h)-1}{h^{2}}=\lim _{h \rightarrow 0+} \frac{\Omega_{k-1}}{P_{n}^{\lambda}(1) h^{2} D(h)} \int_{0}^{h}\left[P_{n}^{\lambda}(\cos \theta)-P_{n}^{\lambda}(1)\right](\sin \theta)^{2 \lambda} d \theta .
$$


Mit der Beziehung ([9, S. 27) und $[8,1142])$

$$
\lim _{h \rightarrow 0+} \frac{P_{n}^{\lambda}(1)-P_{n}^{\lambda}(\cos h)}{P_{n}^{\lambda}(1)(1-\cos h)}=\frac{n(n+2 \lambda)}{2 \lambda+1}
$$

folgt dann

$$
\lim _{h \rightarrow 0+} \frac{B_{n}{ }^{\lambda}(h)-1}{h^{2}}=-\frac{n(n+2 \lambda)}{2(2 \lambda+3)} .
$$

Daher folgt die Gleichung (4.2.21) aus (4.2.22) mit Teil a) von Satz 3.1.

Ist umgekehrt (4.2.21) vorausgesetzt, dann folgt aus der Gleichung

$$
\begin{aligned}
& \frac{M_{h}(f ; \cdot)-f(\cdot)}{h^{2}}-g(\cdot) \\
& =\frac{2(2 \lambda+3)}{h^{2} D(h)} \int_{0}^{h}(\sin \eta)^{2 \lambda} d \eta \int_{0}^{\eta}\left[M_{\tau}(g ; \cdot)-g(\cdot)\right] D(\tau)(\sin \tau)^{-2 \lambda} d \tau \\
& +g(\cdot)\left[j_{M}(h)-1\right],
\end{aligned}
$$

wobei

(4.2.26) $\quad j_{M}(h)=\frac{2(2 \lambda+3)}{h^{2} D(h)} \int_{0}^{h}(\sin \eta)^{2 \lambda} d \eta \int_{0}^{\eta} D(\tau)(\sin \tau)^{-2 \lambda} d \tau$

und $\lim _{h \rightarrow 0+} j_{M}(h)=1$. Daraus folgt für $h \rightarrow 0+$

$$
\left\|\frac{M_{h} f-f}{h^{2}}-g\right\| \leq j_{M}(h) \sup _{0<\tau \leq h}\left\|M_{\tau} g-g\right\|+\left|j_{M}(h)-1\right|\|g\|=o(1),
$$

womit der Satz bewiesen ist.

Bemerkung. Ist die Funktion $f$ aus $S^{3}$ überall in einer Umgebung des Punktes $x \in S^{3}$ definiert und $f \in \mathbb{L}^{1}\left(S^{3}\right)$, dann definiert V. L. Shapiro ([66, S. 16]) den verallgemeinerten Laplace-BeltramiOperator $\Delta_{1}$ im Punkte $x$ durch

$$
\Delta_{1} f(x)=\lim _{h \rightarrow 0+} \frac{2\left\{M_{h}(f ; x)-f(x)\right\}}{\sin ^{2}(h / 2)},
$$

falls der Grenzwert existiert. $\Delta_{1}$ ist eine Verallgemeinerung des Laplace-Beltrami-Operators $\Delta$, denn für jede zweimal stetig in $D(x, h), h>0$, differenzierbare Funktion existiert $\Delta_{1} f(x)$ und ist gleich $\Delta f(x)([66$, S. 18]). Es gilt, wie man sofort sieht, 


$$
\lim _{h \rightarrow 0+} \frac{M_{h}(f ; x)-f(x)}{h^{2}}=\frac{1}{8} \Delta_{1} f(x) .
$$

Der Grenzwert auf der linken Seite der letzten Zeile unterscheidet sich also nur um einen konstanten Faktor von $\Delta_{1} f(x)$. Die linke Seite definiert also (bis auf einen konstanten Faktor) ebenfalls den Operator $\Delta_{1}$. Diese Definition kann man nun für höhere Dimensionen $(k \geq 3)$ beibehalten. Die Formel (4.2.22) im Satz 4.2.4 sagt dann aus, daß der verallgemeinerte Laplace-Operator $\Delta_{1} f$ in $\operatorname{der}$ Norm des Raumes $\boldsymbol{X}$ existiert.

Wir kommen nun $z u$ den Mitteln $S_{h}$, die durch (4.2.8) definiert sind. Analog zu Satz 4.2.4 gilt hier

Satz 4.2.5. Sind die Funktionen $f$ und $g$ aus $\boldsymbol{X}$, dann sind die folgenden Aussagen äquivalent:

$$
\begin{aligned}
& -\frac{n(n+2 \lambda)}{2(2 \lambda+1)} Y_{n}(f ; x)=Y_{n}(g ; x) ; \\
& \lim _{h \rightarrow 0}\left\|\frac{S_{h} f-f}{h^{2}}-g\right\|=0 .
\end{aligned}
$$

Bevor wir den Satz beweisen, wollen wir noch einiges über die Mittel $S_{h} f$ zusammenstellen. Die Mittel $S_{h}(f ; x)$ bilden kein singuläres Integral vom Typ (1.23). Die Integration in (4.2.8) wird nur über den Rand des Gebietes $D(x, h) \subset S^{k}$ durchgeführt und der Rand besitzt auf der $(k-1)$-dimensionalen Fläche $S^{k}$ das Maß Null. $S_{h}(f ; x)$ besitzt aber die wichtigen Eigenschaften (4.2.10) bis (4.2.12). Nach (4.2.11) ist $S_{h}$ für jedes $h>0$ eine beschränkte, lineare Transformation von $\boldsymbol{X}$ in $\boldsymbol{X}$. Erfüllt eine beliebige, beschränkte, lineare Transformation $T$ von $\boldsymbol{X}$ in sich die Bedingung $Y_{n}(T f ; x)=t_{n} Y_{n}(f ; x)$, $n=0,1, \cdots$, wobei $\left\{t_{n}\right\}$ eine Folge von komplexen Zahlen ist, dann heißt $T$ eine Multiplikatoren-Transformation oder auch FaktorfolgenTransformation. Der Begriff eines Multiplikators wird hier in Abschnitt 5.1 genau diskutiert. Es gilt die Aussage, daß jede Multiplikatoren-Transformation von $\boldsymbol{L}^{1}\left(S^{k}\right)$ in $\boldsymbol{L}^{1}\left(S^{k}\right)$ für jedes $f \in \boldsymbol{L}^{1}\left(S^{k}\right)$ als Faltung von $f$ mit einem zonalen $\mathrm{Maß} \mu \in \boldsymbol{M}_{\boldsymbol{\lambda}}$ dargestellt werden kann ([30, S. 261]). Die Faltung ist durch (1.18) gegeben. 
Wegen (4.2.10) ist $S_{h} f$ für jedes $h>0$ eine MultiplikatorenTransformation und daher darstellbar als Faltung (1.18) für alle $f \in \boldsymbol{L}^{1}\left(S^{k}\right)$. Da alle $f \in \mathbb{L}^{p}\left(S^{k}\right), 1<p<\infty$, oder $f \in \boldsymbol{C}\left(S^{k}\right)$ ebenfalls in $\boldsymbol{L}^{1}\left(S^{k}\right)$ enthalten sind, gilt für alle $f \in \mathbb{X}$, daß $S_{h} f$ als Faltung dargestellt werden kann. Dies ist eine Verallgemeinerung des Begriffs eines singulären Integrals vom Typ (1.23). Dort wurde die Faltung mit einer zonalen $\boldsymbol{L}^{1}$-Funktion durchgeführt, also mit einem absolut stetigen zonalen $\mathrm{Maß}$, während $S_{h} f$ die Faltung mit einem nicht absolut stetigen zonalen Maß aus $\boldsymbol{M}_{\lambda}$ bedeutet. $S_{h} f$ besitzt außerdem die Integraldarstellung (4.2.8).

Beweis von Satz 4.2.5: Aus den Eigenschaften (4.2.10), (4.2.11) und (4.2.12) der Mittel $S_{h}(f ; x)$ ist sofort ersichtlich, daß man bei dem Schluß von der Aussage (4.2.29) auf (4.2.28) so vorgehen kann wie im Teil a) von Satz 3.1. Man erhält dann hier

$$
\lim _{h \rightarrow 0+} \frac{P_{n}^{\lambda}(\cos h)-P_{n}^{\lambda}(1)}{P_{n}^{\lambda}(1) h^{2}} Y_{n}(f ; x)=Y_{n}(g ; x) \quad(n=0,1,2, \cdots) .
$$

Nun gilt wegen (4.2.23)

$$
\text { (4.2.30) } \lim _{h \rightarrow 0+} \frac{P_{n}^{\lambda}(\cos h)-P_{n}{ }^{\lambda}(1)}{P_{n}^{\lambda}(1) h^{2}}=-\frac{n(n+2 \lambda)}{2(2 \lambda+1)}
$$

und hieraus folgt (4.2.28). Setzt man umgekehrt (4.2.28) voraus, dann folgt mit Hilfe der Gleichung (4.2.14) ähnlich wie im Beweis des Satzes 4.2.4 die Beziehung

$$
\begin{array}{r}
\frac{S_{h}(f ; \cdot)-f(\cdot)}{h^{2}}-g(\cdot)=\frac{2(2 \lambda+1)}{h^{2} \Omega_{k-1}} \int_{0}^{h}\left[M_{\tau}(g ; \cdot)-g(\cdot)\right] D(\tau)(\sin \tau)^{-2 \lambda} d \tau \\
+g(\cdot)\left[j_{S}(h)-1\right],
\end{array}
$$

wobei

$$
j_{S}(h)=\frac{2(2 \lambda+1)}{h^{2} \Omega_{k-1}} \int_{0}^{h} D(\tau)(\sin \tau)^{-2 \lambda} d \tau, \quad \lim _{h \rightarrow 0+} j_{S}(h)=1 .
$$

Damit folgt wie im Beweis von Satz 4.2.4 die Aussage (4.2.29).

Bemerkung. Der Grenzwert $\left(S_{h} f-f\right) / h^{2}$ ist im wesentlichen wieder eine Verallgemeinerung des Laplace-Operators auf der Kugel. Für $k=3$ definiert W. Rudin [61, S. 288] einen verallgemeinerten Laplace-Beltrami-Operator $\Delta_{2}$ im Punkte $x \in S^{3}$ durch 


$$
\Delta_{2} f(x)=\lim _{h \rightarrow 0+} \frac{S_{h}(f ; x)-f(x)}{\sin ^{2}(h / 2)},
$$

falls der Grenzwert existiert. Man sieht, daß dieser Operator auch für höhere Dimensionen definiert werden kann und mit $\lim _{h \rightarrow 0+}\left[S_{h}(f ; x)\right.$ $-f(x)] / h^{2}$ bis auf eine Konstante übereinstimmt. Der Operator $\Delta_{2}$ ist ein Analogon zur Riemann-Ableitung zweiter Ordnung (siehe [15] und $[19$, Ch. II]) für Funktionen, die auf der reellen Achse definiert sind.

Entsprechend dem Satz 4.1.3 erhält man hier

Satz 4.2.6. Für ein $f \in \boldsymbol{X}$ sind folgende Aussagen äquivalent:

$$
\begin{array}{ll}
\left\|M_{h} f-f_{\|}\right\|=O\left(h^{2}\right) & (h \rightarrow 0+) ; \\
\left\|S_{h} f-f\right\|=O\left(h^{2}\right) & (h \rightarrow 0+) ; \\
f \in \mathcal{H}(\boldsymbol{X} ;-n(n+2 \lambda)) . &
\end{array}
$$

Beweis: Der Satz wird durch Ringschluß bewiesen. Mit Teil b) von Satz 3.1 schließt man von (4.2.31) auf (4.2.33). Im Beweis von Satz 4.2.4 erhielten wir (siehe Gleichung (4.2.24)) $\psi(n)=$ $-n(n+2 \lambda) /(2(2 \lambda+3))$, d.h. $f \in \mathcal{H}(\boldsymbol{X} ;-n(n+2 \lambda))$.

Aus (4.2.33) soll auf (4.2.32) geschlossen werden. Dies soll hier nur für den Raum $L^{1}\left(S^{k}\right)$ durchgeführt werden. Die Beweise für die anderen Räume sind ähnlich, jedoch einfacher. Die Mittel eines Maßes $\mu \in \boldsymbol{M}\left(S^{k}\right)$ sind definiert durch

$$
M_{\tau}(d \mu ; x)=\frac{1}{D(\tau)} \int_{D(x, \tau)} d_{\mu}(y) \quad(\tau>0) .
$$

Es folgt, daß sie überall existieren, zu $\boldsymbol{L}^{1}\left(S^{k}\right)$ gehören und $\left\|M_{\tau} d_{\mu}\right\|_{1}$ $\leq\|\mu\|_{M}$ ist. Außerdem ist $M_{\tau} d_{\mu}$ eine stark stetige, vektorwertige Funktion von $0<\tau<\pi$ in $\boldsymbol{L}^{1}\left(S^{k}\right)$. Weiterhin gilt ähnlich (4.2.6)

$$
Y_{n}\left(M_{\tau} d_{\mu} ; x\right)=B_{n}{ }^{\lambda}(\tau) Y_{n}\left(d_{\mu} ; x\right) \quad(n=0,1, \cdots)
$$

und, falls $-n(n+2 \lambda) Y_{n}(f ; x)=2(2 \lambda+3) Y_{n}(d \mu ; x) \quad$ (d.h. $f \in \mathcal{H}\left(\boldsymbol{L}^{1} ;\right.$ $-n(n+2 \lambda)))$, folgt wie in Lemma 4.2 .3 fast überall

$$
S_{h}(f ; \cdot)-f(\cdot)=\frac{2(2 \lambda+3)}{\Omega_{k-1}} \int_{0}^{h} M_{\tau}\left(d_{\mu} ; \cdot\right) D(\tau)(\sin \tau)^{-2 \lambda} d \tau,
$$


wobei das Integral im Bochnerschen Sinne existiert. Man erhält

$$
\left\|S_{h} f-f\right\|_{1} \leq \frac{2(2 \lambda+3)}{\Omega_{k-1}}\|\mu\|_{M} \int_{0}^{h} D(\tau)(\sin \tau)^{-2 \lambda} d \tau=O\left(h^{2}\right) \quad(h \rightarrow 0+),
$$

womit (4.2.32) für $\boldsymbol{X}=\boldsymbol{L}^{1}\left(S^{k}\right)$ bewiesen ist.

Setzt man (4.2.32) voraus, dann erhält man nach (4.2.9)

$$
\left\|M_{\tau} f-f\right\|=\left\|\frac{\Omega_{k-1}}{D(\tau)} \int_{0}^{\tau}(\sin h)^{2 \lambda}\left[S_{h} f-f\right] d h\right\|=O\left(\tau^{2}\right) \quad(\tau \rightarrow 0+),
$$

womit der Satz ganz bewiesen ist.

Die Ergebnisse der Abschnitte 4.1 und 4.2 lassen sich zusummenfassen in der

Folgerung 4.2.7. Für ein $f \in \boldsymbol{X}$ sind folgende Aussagen äquivalent :

a) $f \in \mathscr{H}(\boldsymbol{X} ;-n(n+2 \lambda))$;

b) für $f$ gilt die Darstellung (4.1.25) bzw. (4.1.26);

c) $\left\|W_{t} f-f\right\|=O(t)$

$(t \rightarrow 0+) ;$

d) $\left\|S_{h} f-f\right\|=O\left(h^{2}\right)$

$(h \rightarrow 0+)$;

e) $\left\|M_{h} f-f\right\|=O\left(h^{2}\right)$

$(h \rightarrow 0+)$.

Der Beweis ergibt sich aus einer Zusammenfassung der Sätze 4.1.3 und 4.2.6 und Lemma 4.1.5. Analoge Ergebnisse erhält man bei Entwicklungen in mehrdimensionalen Fourierreihen für Funktionen, die auf $T^{k}=\left\{x ; x \in E^{k},-\pi<x_{j} \leq \pi, j=1,2, \cdots, k\right\}$ definiert sind (s. [54]).

\section{3 Das singuläre Integral von Abel-Poisson.}

Dieses Integral wurde schon im Kapitel 2 (2.1.10) definiert durch

$$
A_{r}(f ; x)=\frac{1}{\Omega_{k}} \int_{s^{k}} \frac{1-r^{2}}{\left(1-2 r(x, y)+r^{2}\right)^{\lambda+1}} f(y) d s(y)
$$

für alle $f \in \boldsymbol{X}$ und $0 \leq r<1$. Der Kern

$$
p_{r}(u)=\frac{1-r^{2}}{\left(1-2 r t+r^{2}\right)^{\lambda+1}}
$$

ist nicht negativ (siehe (2.1.16)), besitzt die Darstellung (1.4) und die Eigenschaft (2.1.17). Das Analogon $z u$ (4.1.5) lautet hier mit $r=e^{-t}$ 


$$
\begin{aligned}
\frac{1}{\Omega_{k}} \int_{S^{k}} p_{r_{1}}[(x, y)] p_{r_{2}}[(y, z)] d s(y)= & p_{r_{1} \cdot r_{2}}[(x, z)] \\
& \left(x, z \in S^{k} ; 0 \leq r_{1}, r_{2}<1\right) .
\end{aligned}
$$

Weiter gelten (siehe Satz 2.1.1) für alle $f \in \boldsymbol{X}$ die Beziehungen $\left\|A_{r} f\right\| \leq\|f\|, 0 \leq r<1$, und $\lim _{r \rightarrow 1-}\left\|A_{r} f-f\right\|=0$. Aus der Darstellung (2.1.9) folgt

$$
Y_{n}\left(A_{r} f ; x\right)=r^{n} Y_{n}(f ; x) \quad(n=0,1, \cdots) .
$$

$A_{r}(f ; x)$ bildet ein singuläres Integral vom Typ (1.23), wenn man $\rho=1 /(1-r), r \rightarrow 1-$, setzt. Setzt man $r=e^{-t}$ und $A_{r}(f ; x)=\left(V_{t} f\right)(x)$ für alle $f \in \boldsymbol{X}$, dann bildet $\left\{V_{t} ; 0 \leq t<\infty\right\}$ eine stark stetige Halbgruppe von positiven Kontraktionsoperatoren des Klasse $\left(C_{0}\right)$ von $\boldsymbol{X}$ in sich im Sinne von Abschnitt 4.1.

Satz 4.3.1. Für zwei Funktionen $f$ und $g \in \boldsymbol{X}$ sind die folgenden Aussagen äquivalent :

$$
\begin{aligned}
& -n Y_{n}(f ; x)=Y_{n}(g ; x) \quad(n=0,1, \cdots) ; \\
& \lim _{r \rightarrow 1-}\left\|\frac{A_{r} f-f}{1-r}-g\right\|=0 .
\end{aligned}
$$

Beweis: Eine Anwendung des Satzes 3.1, Teil a), zeigt, daß wegen

$$
\lim _{r \rightarrow 1-} \frac{r^{n}-1}{1-r}=-n \quad(n=0,1, \cdots)
$$

aus (4.3.6) die Aussage (4.3.5) folgt.

Aus (4.3.5) ergibt sich wie im Beweis von Satz 4.1.2 (es ist nur $-n(n+2 \lambda)$ durch $-n$ zu ersetzen) die Beziehung (vgl. Abschnitt 4 in [5] für $S^{2}$ )

$$
A_{r} f-f=\int_{r}^{1} A_{\rho} g \frac{d \rho}{\rho}
$$

und damit

$$
\frac{A_{r} f-f}{1-r}-g=\frac{1}{1-r} \int_{r}^{1}\left[A_{\rho} g-g\right] \frac{d \rho}{\rho}-\left(1+\frac{\log r}{1-r}\right) g .
$$

Daraus folgt für $r \rightarrow 1-$ 


$$
\left\|\frac{A_{r} f-f}{1-r}-g\right\| \leq \frac{1}{r} \sup _{r \leq \rho<1}\left\|A_{\rho} g-g\right\|+\left|1+\frac{\log r}{1-r}\right|\|g\|=o(1),
$$

womit (4.3.6) gilt.

Eine weitere äquivalente Aussage zu (4.3.5) oder (4.3.6) bildet

Lemma 4.3.2. Sind $f$ und $g$ aus $\boldsymbol{X}$, dann gilt die Beziehung (4.3.5) genau dann, wenn $f$ darstellbar ist durch

$$
f(x)=Y_{0}(f ; x)-\frac{1}{\Omega_{k}} \int_{s^{k}} h(1,[(x, y)]) g(y) d s(y)
$$

mit

$$
h(1, t)=\int_{0}^{1}\left[\frac{1-r^{2}}{\left(1-2 r t+r^{2}\right)^{\lambda+1}}-1\right] \frac{d r}{r}
$$

und

$$
\begin{aligned}
& |h(1, \cos \theta)| \leq A[\sin (\theta / 2)]^{-2 \lambda} \\
& \left.|| h(1, \cos \theta)\right|_{q, \lambda}<\infty \text { für } 1 \leq q<(2 \lambda+1) / 2 \lambda .
\end{aligned}
$$

Dieses Lemma wurde von P. L. Butzer und Cl. Müller [21] bewiesen. B. Muckenhoupt und E. M. Stein [49, S. 80/81] haben gezeigt, daß die Funktion $h(1, t)$, definiert durch (4.3.10), die Bedingungen (4.3.11) erfüllt und die Gegenbauerentwicklung

$$
h(1, t) \sim \sum_{n=1}^{\infty} \frac{1}{n} \frac{n+\lambda}{\lambda} P_{n}^{\lambda}(t)
$$

besitzt. Daher folgt aus (4.3.5) die Darstellung (4.3.9) nach dem Eindeutigkeitssatz. Die Umkehrung gilt auf Grund des Faltungssatzes. Für $2 \pi$-periodische Funktionen $f(\phi), 0 \leq \phi<2 \pi$, geht (4.3.9) über in

$$
f(\phi)=\frac{1}{2 \pi} \int_{-\pi}^{\pi} f\left(\phi^{\prime}\right) d \phi^{\prime}-\frac{1}{2 \pi} \int_{-\pi}^{\pi} \log \left[1-\cos \left(\phi-\phi^{\prime}\right)\right] g\left(\phi^{\prime}\right) d \phi^{\prime} .
$$

Entsprechend zu Satz 4.1.3 und Lemma 4.1.5 hat man

Satz 4.3.3. Für ein festes $f \in \boldsymbol{X}$ sind die folgenden Aussagen äquivalent :

a) $f \in \mathcal{H}(\boldsymbol{X} ;-n)$;

b) $\left\|A_{r} f-f\right\|=O(1-r)$ $(r \rightarrow 1-)$ 
c) im Falle $\boldsymbol{X}=\boldsymbol{C}\left(S^{k}\right)$ besitzt $f(x)$ überall die Darstellung (4.3.9) mit $g \in \boldsymbol{L}^{\infty}\left(S^{k}\right)$,

im Falle $\boldsymbol{X}=\boldsymbol{L}^{p}\left(S^{k}\right), 1<p<\infty$, besitzt $f(x)$ fast überall die Darstellung (4.3.9),

ist $\boldsymbol{X}=\boldsymbol{L}^{1}\left(S^{k}\right)$, dann existiert ein $M a \beta \mu \in \boldsymbol{M}\left(S^{k}\right)$, so daß fast überall gilt

$$
f(x)=Y_{0}(f ; x)-\frac{1}{\Omega_{k}} \int_{S^{k}} h(1,[(x, y)]) d_{\mu}(y) .
$$

Beweis: Aus b) folgt a) nach Teil b) des Saturationssatzes 3.1, wenn man (4.3.7) berücksichtigt. Für $f \in L^{p}\left(S^{k}\right), 1<p<\infty$, folgt b) aus a) mit der Identität (4.3.8). Ist $f \in C\left(S^{k}\right)$, dann gilt (4.3.8) mit $g \in \boldsymbol{L}^{\infty}\left(S^{k}\right)$. Für $\boldsymbol{X}=\boldsymbol{L}^{1}\left(S^{k}\right)$ kann man aus a) wie im Beweis von Satz 4.1.3 zeigen, daß fast überall gilt

mit

$$
A_{r} f(; \cdot)-f(\cdot)=\int_{r}^{1}\left[p_{\rho} * d \mu\right](\cdot) \frac{d \rho}{\rho}
$$

$$
\left[p_{\rho} * d \mu\right](x)=\frac{1}{\Omega_{k}} \int_{S^{k}} p_{\rho}[(x, y)] d \mu(y) .
$$

Damit folgt b) aus a) auch für $\boldsymbol{X}=\boldsymbol{C}\left(S^{k}\right)$ und $\boldsymbol{X}=\mathbb{L}^{1}\left(S^{k}\right)$. Die Äquivalen $z$ von a) und c) folgt in allen Räumen $\boldsymbol{X}$ wie im Beweis von Lemma 4.3.2, womit Satz 4.3.3 bewiesen ist.

Folgerung 4.3.3. Die Saturationsordnung des singulären Integrals $A_{r}(f ; x)$ ist $O(1-r)$ für $r \rightarrow 1$ - und seine Saturationsklasse ist gegeben durch $\mathcal{H}(\boldsymbol{X} ;-n)$.

Im Falle der Funktionen, die auf dem Einheitskreis definiert sind, ist die Saturationsklasse des singulären Integrals gegeben durch alle Funktionen, deren konjugierte Funktion $\tilde{f}$ (s. [74, Ch. III]) einer Lipschitzbedingung genügt d.h. $\|\tilde{f}(\theta+h)-\tilde{f}(\theta)\|=O(h), h \rightarrow 0+$, ([13], [11], [70, I], [19, Sec. 2.4]). Ähnliche Charakterisierungen gelten für zonale Funktionen auf $S^{k}$, was im Abschnitt 5.4. dieser Arbeit bewiesen wird.

Als weitere Anwendung betrachten wir ein singuläres Integral, welches dieselbe Saturationsklasse wie $A_{r}(f ; x)$ besitzt. 


\section{4. Das Summationsverfahren von Cesàro.}

Dieser Summationsprozeß wurde in Abschnitt 2.1 in Formel (2.1.2) definiert durch

$$
\sigma_{N}^{\alpha}(f ; x)=\left(A_{N}^{\alpha}\right)^{-1} \sum_{n=0}^{N} A_{N-n}^{\alpha} Y_{n}(f ; x)
$$

und dort schon ausführlich untersucht. Daher beginnen wir hier mit dem Saturationssatz für die Mittel $\sigma_{N}^{\alpha}$.

Setzt man $\rho=N$ und $\varphi(\rho)=N^{-1}$, dann gilt wegen (s. [74, S. 77])

$$
A_{N}^{\alpha}=\frac{N^{\alpha}}{\Gamma(\alpha+1)}\left\{1+O\left(\frac{1}{N}\right)\right\}
$$

die Beziehung (s. [20, S. 388])

$$
\lim _{N \rightarrow \infty} N^{-1}\left[1-A_{N-n}^{\alpha} /\left(A_{N}^{\alpha}\right)\right]=-\alpha n \quad(n=0,1, \cdots) .
$$

Falls nicht anders angegeben, wird von jetzt ab immer $\alpha>\lambda$ vorausgesetzt.

Satz 4.4.1. Für ein $f$ aus $\boldsymbol{X}$ gilt

$$
\left\|\sigma_{N}^{\alpha} f-f\right\|=O\left(N^{-1}\right)
$$

genau dann, falls $f \in \mathcal{H}(\boldsymbol{X} ;-\alpha n)$ ist.

Beweis: Aus (4.4.3) folgt wegen (4.4.2) mit Teil b) des Satzes 3.1 wieder $f \in \mathcal{H}(\boldsymbol{X} ;-\alpha n)$. Um die Umkehrung zu beweisen, benutzen wir die Identität (z.B. [74, S. 269])

$$
\begin{aligned}
& \sigma_{N}^{\alpha+1}(f ; x)-\sigma_{N-1}^{\alpha+1}(f ; x) \\
& \quad=\frac{\alpha+1}{N(N+\alpha+1)}\left(A_{N}^{\alpha}\right)^{-1} \sum_{n=0}^{N} A_{N-n}^{\alpha} n Y_{n}(f ; x) \quad\left(x \in S^{k}\right) .
\end{aligned}
$$

Nehmen wir speziell den Raum $\boldsymbol{X}=\boldsymbol{C}\left(S^{k}\right)$, dann existiert nach Voraussetzung eine Funktion $g \in \boldsymbol{L}^{\infty}\left(S^{k}\right)$, so daß gilt $-\alpha n Y_{n}(f ; x)=$ $Y_{n}(g ; x), n=0,1,2, \cdots$. Hiermit folgt aus (4.4.4)

$$
\sigma_{N}^{\alpha_{+1}}(f ; x)-\sigma_{N-1}^{\alpha_{+}}(f ; x)=-\frac{(\alpha+1)}{N(N+\alpha+1)} \sigma_{N}^{\alpha}(g ; x),
$$

und daraus 


$$
\begin{aligned}
\sigma_{N_{1}}^{\alpha+1}(f ; x)-\sigma_{N}^{\alpha+1}(f ; x) & =\sum_{l=N+1}^{N_{1}}\left[\sigma_{l}^{\alpha+1}(f ; x)-\alpha_{l-1}^{\alpha+1}(f ; x)\right] \\
& =-\frac{\alpha+1}{\alpha} \sum_{l=N+1}^{N_{1}} \frac{1}{l(l+\alpha+1)} \sigma_{l}^{\alpha}(g ; x) .
\end{aligned}
$$

Also gilt

$$
\begin{aligned}
\left\|\sigma_{N_{1}}^{\alpha+1} f-\sigma_{N}^{\alpha_{+1}} f\right\|_{C} & \leq \frac{\alpha+1}{\alpha} \sum_{l=N+1}^{N_{1}} \frac{1}{l(l+\alpha+1)}\left\|\sigma_{l}^{\alpha} g\right\|_{C} \\
& \leq M_{\alpha} \frac{\alpha+1}{\alpha}\|g\|_{\infty} \int_{N+1}^{\infty} \frac{d x}{x(x+\alpha+1)}=O\left(N^{-1}\right)
\end{aligned}
$$

wobei $M_{c}$ die Konstante aus (2.1.6) und die rechte Seite unabhängig von $N_{1}$ ist. Daher kann man dort den Grenzübergang $\lim _{N_{1} \rightarrow \infty}$ durchführen und man erhält wegen (2.1.8) die Beziehung

$$
\left\|f-\sigma_{N}^{\alpha+1} f\right\|_{C}=O\left(N^{-1}\right)
$$

$(N \rightarrow \infty)$.

Damit ist (4.4.3) mit $(\alpha+1)$ an Stelle von $\alpha$ bewiesen. Mit der folgenden Identität kann man leicht von $\alpha+1$ auf $\alpha$ schließen. Es gilt (s. [74, S. 269])

$$
\sigma_{N}^{\alpha}(f ; x)=\sigma_{N}^{\alpha_{+1}}(f ; x)+\frac{1}{N+\alpha+1}\left(A_{N}^{\alpha}\right)^{-1} \sum_{n=0}^{N} A_{N-n}^{\alpha} n Y_{n}(f ; x)
$$

und damit nach Voraussetzung

$$
\sigma_{N}^{\alpha}(f ; x)=\sigma_{N}^{\alpha+1}(f ; x)-\frac{1}{\alpha(N+\alpha+1)} \sigma_{N}^{\alpha}(g ; x) .
$$

Mit (4.4.7) erhält man dann

$$
\left\|\sigma_{N}^{\alpha} f-f\right\|_{C} \leq\left\|\sigma_{N}^{\alpha+1} f-f\right\|_{C}+\frac{1}{\alpha(N+\alpha+1)}\left\|\sigma_{N}^{\alpha} g\right\|_{C}=O\left(N^{-1}\right) \quad(N \rightarrow \infty),
$$

womit (4.4.3) für $\boldsymbol{X}=\boldsymbol{C}\left(S^{k}\right)$ bewiesen ist. Der Beweis in den anderen Räumen verläuft ähnlich. Diese Beweismethode wurde schon in [73], [2], [34] und [24] benutzt. Satz 4.4.1 folgt auch aus den Ergebnissen in [24].

Eine Grenzwertbeziehung wie in Satz 4.3.1 ist auch hier gültig. Man erhält hier

Satz 4.4.2. Für zwei Funktionen $f$ und $g \in \boldsymbol{X}$ sind die folgenden Aussagen äquivalent : 


$$
-\alpha n Y_{n}(f ; x)=Y_{n}(g ; x)
$$$$
(n=0,1, \cdots) \text {; }
$$

$$
\left\|N\left[\sigma_{N}^{\alpha} f-f\right]-g\right\|=o(1)
$$

Beweis: Der Schluß von (4.4.11) auf (4.4.10) ist klar. Zum Beweis der Umkehrung geht man wie im Beweis des Satzes 4.4.1 vor. Aus (4.4.10) folgt mit (4.4.6) und $N_{1} \rightarrow \infty$

$$
N\left[\sigma_{N}^{\alpha+1} f-f\right]=\frac{\alpha+1}{\alpha} N \sum_{l=N+1}^{\infty} \frac{1}{l(l+\alpha+1)} \sigma_{l}{ }^{\alpha} g,
$$

wobei die unendliche Reihe in der Norm konvergiert. Für die Folge $\left\{c_{N}\right\}_{N=1}^{\infty}$, definiert durch

$$
c_{N}=N \sum_{l=N+1}^{\infty} \frac{1}{l(l+\alpha+1)},
$$

gilt nun $\lim _{N \rightarrow \infty} c_{N}=1$. Daher folgt aus

$$
\begin{aligned}
N & {\left[\sigma_{N}^{\alpha+1} f-f\right]-\frac{\alpha+1}{\alpha} g } \\
& =\frac{\alpha+1}{\alpha} N \sum_{l=N+1}^{\infty} \frac{1}{l(l+\alpha+1)}\left[\sigma_{l}{ }^{\alpha} g-g\right]+\frac{\alpha+1}{\alpha}\left[c_{N}-1\right] g
\end{aligned}
$$

sofort für $N \rightarrow \infty$

$$
\begin{aligned}
& \left\|N\left[\sigma_{N}^{\alpha+1} f-f\right]-\frac{\alpha+1}{\alpha} g\right\| \\
& \quad \leq \frac{\alpha+1}{\alpha} c_{N} \sup _{l \geq N+1}\left\|\sigma_{l}{ }^{\text {rs }} g-g\right\|_{1}+\frac{\alpha+1}{\alpha}\left|c_{N}-1\right|\|g\|=o(1)
\end{aligned}
$$

wegen (2.1.8). Weiter folgt aus (4.4.9)

$$
\begin{aligned}
N\left[\sigma_{N}^{\alpha} f-f\right]-g= & \left\{N\left[\sigma_{N}^{\alpha+1} f-f\right]-\frac{\alpha+1}{\alpha} g\right\} \\
& -\frac{N}{\alpha(N+\alpha+1)}\left\{\sigma_{N}^{\alpha} g-g\right\}+\frac{\alpha+1}{\alpha(N+\alpha+1)} g .
\end{aligned}
$$

Dies ergibt mit (4.4.12) und (2.1.8) Aussage (4.4.11).

Für die Cesàro-Mittel von Fourierreihen wurde die Grenzwertziehung (4.4.11) in [20, S. 388] für alle $\alpha>\lambda=0$ bewiesen. Das Ergebnis wird dort durch eine Anwendung eines allgemeinen Satzes für singuläre Integrale gewonnen.

Wir formulieren hier noch den Saturationssatz als 
Folgerung 4.4.3. Das singuläre Integral $\sigma_{N}^{\alpha}(f ; x), \alpha>\lambda$, von Cesàro besitzt in den Räumen $\boldsymbol{X}$ die Saturationsordnung $O\left(N^{-1}\right)$, $N \rightarrow \infty$, und die Saturationsklasse $\mathcal{H}(\boldsymbol{X} ;-\alpha n)$.

Man sieht an den Ergebnissen der Abschnitte 4.3 und 4.4, daß die Saturationsklassen der singulären Integrale von Abel-Poisson und Cesàro übereinstimmen und $\mathrm{da} \beta$ die Klassen gleich sind, die eine Grenzwertbeziehung für eins der Integrale zulassen.

\section{Funktionenklassen auf der Kugel}

\section{1. Das verallgemeinerte singuläre Integral von Weierstraß.}

Das verallgemeinerte singuläre Integral von Weierstraß wurde von S. Bochner [7, S. 84] eingeführt. Für $f \in X$ ist es definiert durch

$$
W_{t}{ }^{\kappa}(f ; x)=\sum_{n=0}^{\infty} e^{-\left[{ }^{n(n+2 \lambda)]^{\kappa} t}\right.} Y_{n}(f ; x) \quad\left(x \in S^{k} ; 0<\kappa \leq 1\right),
$$

wobei die unendliche Reihe absolut und gleichmäßig auf $S^{k}$ für jedes $t>0$ konvergiert. Eine andere Schreibweise ist

$$
W_{t}{ }^{\kappa}(f ; x)=\frac{1}{\Omega_{k}} \int_{S^{k}} w_{t}{ }^{\kappa}[(x, y)] f(y) d s(y)
$$

mit dem Kern $w_{t}{ }^{k}(\cos \theta)$, der dargestellt wird durch die Reihe

$$
w_{t}{ }^{\kappa}(\cos \theta)=\sum_{n=0}^{\infty} e^{-[n(n+2 \lambda)]^{\kappa} t} \frac{n+\lambda}{\lambda} P_{n}{ }^{\lambda}(\cos \theta) \quad(0 \leq \theta \leq \pi) .
$$

Für $\kappa=1$ fällt dieses Integral mit $W_{t}(f ; x)$ zusammen, welches in Abschnitt 4.1 behandelt wurde. Sehr viele Eigenschaften von $W_{t}$ sind auch für $W_{t}{ }^{\kappa}$ gültig. Für $w_{t}{ }^{\kappa}, 0<\kappa \leq 1$, an Stelle von $w_{t}$ gilt wieder (4.1.3) (s. [7] and [9, S. 47]), (4.1.4) und (4.1.5). Weiter hat man für alle $f \in \boldsymbol{X}, 0<\kappa \leq 1$,

$$
\left\|W_{t}{ }^{\kappa} f\right\| \leq\|f\| \quad(t>0), \lim _{t \rightarrow 0+}\left\|W_{t}{ }^{\kappa} f-f\right\|=0 .
$$

Der Beweis läßt sich direkt aus Abschnitt 4.1 übernehmen. Für den Grenzwertsatz und den Saturationssatz benötigt man noch die Faktorfolge

$$
\left\{\psi(n)=-[n(n+2 \lambda)]^{\kappa} ; \quad n=0,1,2, \cdots\right\},
$$


die die $\mathscr{H}$-Klassen bestimmt. $\mathrm{Zu}$ bemerken ist noch, daß die Menge von Operatoren $\left\{W_{t}^{k}, 0 \leq t<\infty\right\}$ für $0<\kappa \leq 1$ eine Halbgruppe von Kontraktionsoperatoren der Klasse $\left(C_{0}\right)$ bildet.

Das Analogon zu den Sätzen 4.1.2 und 4.1.3 lautet

Satz 5.1.1. a) Für zwei Funktionen $f$ und $g$ aus $\boldsymbol{X}$ sind folgende Aussagen äquivalent :

$$
\begin{aligned}
& -[n(n+2 \lambda)]^{\kappa} Y_{n}(f ; x)=Y_{n}(g ; x) ; \\
& \lim _{t \rightarrow 0+}\left\|\frac{W_{t}{ }^{\kappa} f-f}{t}-g\right\|=0 .
\end{aligned}
$$

b) Ist $f \in \boldsymbol{X}$, dann gilt $f \in \mathcal{H}\left(\boldsymbol{X} ;-[n(n+2 \lambda)]^{\kappa}\right)$ genau dann, wenn

$$
\left\|W_{t}{ }^{\kappa} f-f\right\|=O(t)
$$

$$
(t \rightarrow 0+) .
$$

Es bleibt noch übrig, Eigenschaften der Klassen $\mathscr{H}(\boldsymbol{X} ;-[n(n+$ $2 \lambda)]^{\kappa}$ ) anzugeben, und die Klassen der Funktionen $f$ zu untersuchen, die durch (5.1.6) oder (5.1.7) bestimmt sind und in den Fällen $\boldsymbol{L}^{1}\left(S^{k}\right)$ und $\boldsymbol{C}\left(S^{k}\right)$ in $\mathcal{H}\left(\boldsymbol{X} ;-[n(n+2 \lambda)]^{\kappa}\right)$ enthalten sind. Da ähnliche Funktionenklassen bei der Behandlung des verallgemeinerten singulären Integrals von Abel-Poisson auftreten, wird diese Frage dort untersucht.

\section{2. Das verallgemeinerte singuläre Integral von Abel-Poisson.}

Als Spezialfall der allgemeinen Untersuchungen von S. Bochner ([9, S. 43-47]) definieren wir das verallgemeinerte singuläre Integral von Abel-Poisson für $0<\gamma \leq 1$ durch

$$
V_{t}^{\gamma}(f ; x)=\sum_{n=0}^{\infty} \exp \left[-n^{\gamma} t\right] Y_{n}(f ; x) \quad(f \in X, t>0) .
$$

Der Fall $\gamma=1$ ist wieder das spezielle Integral von Abel-Poisson, das wir in Abschnitt 4.3 eingeführt haben. Es wurde $r=e^{-t}$ gesetzt ; diese Bezeichnungsweise wurde hier beibehalten. Man kann $V_{t}^{\gamma}(f ; x)$ ebenfalls als Faltungsintegral mit einem Kern $v_{t}^{\gamma}$ schreiben, der gegeben ist durch

$$
v_{i}^{\gamma}(\cos \theta)=\sum_{n=0}^{\infty} \exp \left[-n^{\gamma} t\right] \frac{n+\lambda}{\lambda} P_{n}^{\lambda}(\cos \theta) \quad(0 \leq \theta \leq \pi) .
$$

Auch für diesen Kern gilt wieder $v_{t}^{\gamma}(\cos \theta) \geq 0,0<\gamma \leq 1, t>0$, 
$0 \leq \theta \leq \pi$. Diese wichtige Eigenschaft wurde in [9, S. 43-47] und [45] bewiesen. Sie folgt aus der Positivität für $\gamma=1$ (Formel (4.3.2)) und der Tatsache, daß die Funktion $y^{\gamma}$ "vollständig monoton" auf $(0, \infty)$ für $0<\gamma<1$ ist. Für den Kern $v_{t}^{\gamma}$ gilt auch (2.1.17) und (4.3.3). Die Operatoren $V_{t}^{\gamma}$ bilden ein Summationsverfahren, denn für alle $f$ aus $\boldsymbol{X}$ gilt

$$
\left\|V_{t}^{\gamma} f\right\| \leq\|f\| \quad(t>0) ; \lim _{t \rightarrow 0+}\left\|V_{t}^{\gamma} f-f\right\|=0 .
$$

Die Familie $\left\{V_{t}^{\gamma}, 0 \leq t<\infty\right\}$ bildet ebenfalls eine Halbgruppe von Kontraktionsoperatoren der Klasse $\left(C_{0}\right)$. Die Faktorfolge $\{\psi(n)\}$ aus (3.2) ist hier gegeben durch

$$
\left\{\psi(n)=-n^{\gamma}, \quad n=0,1,2, \cdots\right\} .
$$

Satz 5.2.1. a) Sind $f$ und $g$ Funktionen aus $\boldsymbol{X}$, dann sind folgende Behauptungen äquivalent :

$$
\begin{aligned}
& -n^{\gamma} Y_{n}(f ; x)=Y_{n}(g ; x) \quad(n=0,1, \cdots) ; \\
& \lim _{t \rightarrow 0+}\left\|\frac{V_{t}^{\gamma} f-f}{t}-g\right\|=0 .
\end{aligned}
$$

b) Für ein $f \in \boldsymbol{X}$ sind die folgenden Aussagen äquivalent :

$$
\begin{aligned}
& f \in \mathcal{H}\left(\boldsymbol{X} ;-n^{\gamma}\right) ; \\
& \left\|V_{t}{ }^{\gamma} f-f\right\|=O(t)
\end{aligned}
$$

$$
(t \rightarrow 0+) .
$$

Wir untersuchen nun die Klassen $\mathscr{H}\left(\boldsymbol{X} ;-n^{\gamma}\right)$ für alle $\gamma>0$ und $\mathscr{H}\left(\boldsymbol{X} ;-[n(n+2 \lambda)]^{\kappa}\right), \kappa>0$. Unter anderem erhalten wir, daß diese Klassen für $\gamma=2 \kappa$ übereinstimmen, d.h. die Saturationsklasse von $V_{t}^{2 \kappa}, 0<\kappa \leq 1 / 2$, ist gleich der Saturationsklasse von $W_{t}^{\kappa}$ für diese Werte von $\kappa$.

\section{3 Klassen von Funktionen auf $\boldsymbol{S}^{k}$.}

Die Klassen $\mathscr{H}\left(\boldsymbol{X} ;-n^{\gamma}\right), \gamma>0$, und $\mathscr{H}\left(\boldsymbol{X} ;-[n(n+2 \lambda)]^{\kappa}\right), \kappa>0$, werden hier untersucht. Außerdem betrachten wir die zugehörigen Unterklassen von Funktionen, die in den Sätzen 5.1.1 a) bzw. 5.2.1 a) für die singulären Integrale $W_{t}^{\kappa}$ bzw. $V_{t}^{\gamma}$ auftreten. Es sind dies

$$
\mathcal{H}_{a}(\boldsymbol{X} ; \psi(n))=\left\{f \in \boldsymbol{X} ; g \in \boldsymbol{X} \operatorname{mit} \psi(n) Y_{n}(f ; x)=Y_{n}(g ; x)\right\},
$$


wobei $\psi(n)=-n^{\gamma}, \gamma>0$, bzw. $\psi(n)=-[n(n+2 \lambda)]^{\kappa}, \kappa>0$, ist. Definitionsgemäß gilt $\mathscr{H}_{a}\left(\boldsymbol{L}^{p} ; \psi(n)\right)=\mathscr{H}\left(\boldsymbol{L}^{p} ; \psi(n)\right)$ im Falle $1<p<\infty$. In den Räumen $\boldsymbol{X}=\boldsymbol{L}^{1}\left(S^{k}\right)$ und $\boldsymbol{X}=\boldsymbol{C}\left(S^{k}\right)$ sind die $\mathscr{H}_{a}(\boldsymbol{X} ; \psi(n))$ echte Unterklassen von $\mathscr{H}(\boldsymbol{X} ; \psi(n))$.

Wir benötigen nun den Begriff eines Multiplikators. Sind $\boldsymbol{Y}$ und $\boldsymbol{Z}$ zwei Räume von Funktionen auf $S^{k}$ (hier die Räume $\boldsymbol{C}, \boldsymbol{L}^{p}(1 \leq p$ $\leq \infty), \boldsymbol{M}$, die sonst mit $\boldsymbol{C}\left(S^{k}\right), \boldsymbol{L}^{p}\left(S^{k}\right)$ oder $\mathbb{M}\left(S^{k}\right)$ bezeichnet wurden), dann definiert man einen Multiplikator wie folgt (siehe [4, II, S. 240]) :

Definition. Eine Folge von reellen oder komplexen Zahlen $\left\{a_{n}\right.$; $n=0,1,2, \cdots\}$ heißt ein Multiplikator von $\boldsymbol{Y}$ in $\mathbb{Z}$, falls gilt: Hat $f \in \boldsymbol{Y}$ die Laplaceentwicklung

$$
S(f ; x) \sim \sum_{n=0}^{\infty} Y_{n}(f ; x),
$$

dann existiert ein $g \in \boldsymbol{Z}$ mit der Entwicklung

$$
S(g ; x) \sim \sum_{n=0}^{\infty} \frac{\lambda}{n+\lambda} a_{n} Y_{n}(f ; x) .
$$

Ist $\boldsymbol{Y}$ oder $\boldsymbol{Z}$ gleich $\boldsymbol{M}$ (oder beide), dann ersetzt man in den Formeln (5.3.2) bzw. (5.3.3) $f$ durch $d \mu$ oder $g$ durch $d \nu$ (oder beide). Man schreibt $\left\{a_{n}\right\} \in(\mathbb{Y}, \boldsymbol{Z})$.

Bemerkung. Im Unterschied $\mathrm{zu}$ [4] wurde hier der Faktor $\lambda /(n+\lambda)$ in $S(g ; x)$ hinzugefügt. Dies geschah wegen der Eigenschaft (1.15) der Faltung.

Ist $\boldsymbol{Y}=\boldsymbol{Z}=\boldsymbol{C}$ oder $\boldsymbol{L}^{1}$, dann gilt $\left\{a_{n}\right\} \in(\boldsymbol{C}, \boldsymbol{C})$ oder $\left\{a_{n}\right\} \in\left(\boldsymbol{L}^{1}, \boldsymbol{L}^{1}\right)$ genau dann, wenn die Folge $\left\{a_{n}\right\}$ die Gegenbauer-Stieltjes-Koeffizienten eines Maßes $\mu \in \mathbb{M}_{\lambda}$ sind, d.h. es existiert ein $\mathrm{Ma} \beta \mu \mathbb{M}_{\lambda}$, so daß gilt (vgl. Formel (1.19)) : $a_{n}=\check{\mu}(n), n=0,1,2, \cdots$ (siehe [30, S. 254 u. 261]). Satz 1.5 ergibt $(\boldsymbol{C}, \boldsymbol{C}) \subset\left(\boldsymbol{L}^{p}, \mathbb{L}^{p}\right), 1 \leq p \leq \infty$. Nach dem Satz von Riesz-Fischer ist jede Folge $\left\{a_{n}\right\}$ mit $\left|\lambda a_{n} /(n+\lambda)\right| \leq M$, $n \geq 0$, in $\left(\boldsymbol{L}^{2}, \boldsymbol{L}^{2}\right)$.

Wir wollen noch den Fall $\boldsymbol{Y}=\boldsymbol{Z}=\boldsymbol{M}$ untersuchen. Hier gilt das gleiche Ergebnis wie bei $\boldsymbol{C}$ und $\mathbb{L}^{1}$, wie es auch bei Fourierreihen gültig ist (s. [74, S. 176] und [37, Sec. 20.2]). 
Lemma 5.3.1. Eine notwendige und hinreichende Bedingung dafür, daß eine Folge $\left\{a_{n}, n=0,1,2, \cdots\right\}$ zur Klasse $(\boldsymbol{M}, \boldsymbol{M})$ gehört, ist $d a \beta$ die $a_{n}$ die Gegenbauer-Stieltjes-Koeffizienten eines zonalen Maßes $\mu(\theta)$ sind (d.h. $a_{n}$ besitzt die Darstellung (1.19)).

Der Beweis beruht auf der folgenden Aussage: Die Glieder der Folge $\left\{a_{n}\right\}$ besitzen die Darstellung (1.19) genau dann, falls

a) für die Cesàro-Mittel

gilt

$$
\sigma_{N}^{\alpha}(x)=\left(A_{N}^{\alpha}\right)^{-1} \sum_{n=0}^{N} A_{N-n}^{\alpha} a_{n} P_{n}^{\lambda}(\cos \theta) \quad(\alpha>\lambda)
$$

$$
\left\|\sigma_{N}^{\alpha}\right\|_{1, \lambda} \leq M_{1}
$$$$
(N=0,1, \cdots),
$$

oder b) für die Abel-Mittel

$$
V_{r}(x)=\sum_{n=0}^{\infty} r^{n} a_{n} P_{n}^{\lambda}(\cos \theta)
$$

die Bedingung

$$
\left\|V_{r}\right\|_{1, \lambda} \leq M_{2}
$$

erfüllt ist.

Der Beweis verläuft wie bei Fourierreihen (siehe [74, S. 137 u. $148]$ für a) und [74, S. 149] für b), und zwar die Notwendigkeit von (5.3.4) oder (5.3.5) aus dem Faltungssatz, die Hinlänglichkeit wie im Beweis von Satz 3.1, Teil b) für den Raum $\boldsymbol{L}^{1}\left(S^{k}\right)$. (Für die Fejér-Mittel und $\lambda=1 / 2$ wurde dies schon in [16] bewiesen).

Beweis von Lemma 5.3.1: Ist $\left\{a_{n}\right\} \in(\boldsymbol{M}, \boldsymbol{M})$, dann transformiert $\left\{a_{n}\right\}$ die spezielle Reihe

$$
\sum_{n=0}^{\infty} \frac{n+\lambda}{\lambda} P_{n}^{\lambda}(\cos \theta)
$$

die wegen (1.4), (2.1.17) und der obigen Aussage eine StieltjesReihe ist, in die Stieltjes-Reihe

$$
\sum_{n=0}^{\infty} a_{n} P_{n}{ }^{\lambda}(\cos \theta),
$$

woraus folgt, daß jedes $a_{n}, n=0,1, \cdots$, die Darstellung (1.19) besitzt, womit die eine Richtung der Aussage bewiesen ist. (Die Funktionen $Y_{n}\left(d_{\mu} ; x\right)$ in (5.3.2) und (5.3.3) sind hier die zonalen 
Funktionen $\left.[(n+\lambda) / \lambda] P_{n}{ }^{\lambda}(\cos \theta)\right)$.

Existiert umgekehrt ein zonales $\mathrm{Ma} \beta \mu(\theta)$, so daß (1.19) gilt, dann bildet man die Cesàro-Mittel $\sigma_{N}^{\alpha}(d \nu ; x)=\left(K_{N}^{\alpha} * d \nu\right)(x)$ eines beliebigen Maßes $\nu$ aus $\boldsymbol{M}$. Die Faltung dieser Cesàro-Mittel mit dem zonalen $\mathrm{Maß} \mu$ besitzt die Entwicklung

$$
\begin{aligned}
\left.Y_{n}\left\{\left[K_{N^{*}}^{\alpha} d \nu\right) * d \mu\right] ; x\right\} & =Y_{n}\left[K_{N^{*}}^{\alpha} d \nu ; x\right] \cdot \check{\mu}(n) \frac{\lambda}{n+\lambda} \\
& =\frac{\lambda}{n+\lambda} \check{\mu}(n) \frac{A_{N-n}^{\alpha}}{A_{N}^{\alpha}} Y_{n}(d \nu ; x),
\end{aligned}
$$

d.h. $\left[\left(K_{N}^{\alpha} * d \nu\right) * d \mu\right](x)$ sind die Cesàro-Mittel der Entwicklung

$$
\sum_{n=0}^{\infty} \frac{\lambda}{n+\lambda} \stackrel{v}{\mu}(n) Y_{n}(d \nu ; x),
$$

und es gilt

$$
\left\|\left.\left(K_{N}^{\alpha} * d \nu\right) * d \mu i\right|_{1} \leq\right\| K_{N^{*}}^{\alpha} d \nu\left\|_{1}\right\| \mu\left\|_{M_{\lambda}} \leq M_{\alpha}\right\| \nu\left\|_{M}\right\| \mu \|_{M_{\lambda}} \leq C \quad(N \geq 1)
$$

wegen Satz 1.5, Satz 1.4 und (2.1.6). Aus der obigen Aussage folgt direkt, daß (5.3.6) die Entwicklung eines Maßes aus $M$ ist.

Es gilt also (vgl. auch [39, S. 222-231]).

$$
(\boldsymbol{M}, \boldsymbol{M})=(\boldsymbol{C}, \boldsymbol{C})=\left(\boldsymbol{L}^{1}, \boldsymbol{L}^{1}\right) \subset\left(\boldsymbol{L}^{p}, \boldsymbol{L}^{p}\right) \subset\left(\boldsymbol{L}^{2}, \boldsymbol{L}^{2}\right) \quad(1<p<\infty) .
$$

Als Anwendung von Lemma (5.3.1) beweisen wir

Lemma 5.3.2. Die Folge

$$
\left\{c_{n}{ }^{\kappa}=\frac{n+\lambda}{\lambda}\left(\frac{n}{n+2 \lambda}\right)^{\kappa} ; n=1,2, \cdots ; c_{0}{ }^{\kappa}=0\right\} .
$$

bildet für jedes reelle $\kappa$ die Koeffizienten eines zonalen Maßes aus M.

Beweis. Die Methode ist [71, S, 465] entnommen. Es ist für $n=1,2, \cdots$

$$
\begin{aligned}
\left(\frac{n}{n+2 \lambda}\right)^{\kappa}=\left(\frac{1}{1+(2 \lambda / n)}\right)^{\kappa} & =1+\frac{t_{1}(\kappa, \lambda)}{n}+\frac{t_{2}(\kappa, \lambda)}{n^{2}}+\cdots \\
& +f_{\kappa}^{(2 \lambda+2)}\left(\xi_{n}\right) \frac{(2 \lambda)^{2 \lambda+2}}{(2 \lambda+2) ! n^{2 \lambda+2}} \quad\left(0<\xi_{n}<2 \lambda / n\right),
\end{aligned}
$$

da die Funktion $f_{\kappa}(t)=(1+t)^{-\kappa}$ beliebig oft differenzierbar ist für $t>-1$. Man multipliziert die letzte Gleichung mit $(n+\lambda) / \lambda$. Das 
erste Glied der rechten Seite: $(n+\lambda) / \lambda$, bildet die Koeffizienten eines beschränken Maßes, wie oben gezeigt wurde und die Glieder $(n+\lambda) / n^{\alpha}(\alpha>0)$ sind die Gegenbauer-Koeffizienten einer $\boldsymbol{L}_{\lambda}{ }^{1}$-Funktion (siehe [4, I, S. 202/203]). Der letzte Ausdruck bildet wegen

$$
\begin{aligned}
\sum_{n=1}^{\infty}\left|f_{\kappa}^{(2 \lambda+2)}\left(\xi_{n}\right)\right| \frac{(2 \lambda)^{2 \lambda+2}}{(2 \lambda+2) ! n^{2 \lambda+2}} \frac{n+\lambda}{\lambda}\left|P_{n}^{\lambda}(\cos \theta)\right| \\
\leq \sum_{n=1}^{\infty} \frac{K(\kappa, \lambda)}{n^{2 \lambda+1}}\left|P_{n}^{\lambda}(1)\right| \leq K_{1}(\kappa, \lambda) \sum_{n=1}^{\infty} \frac{1}{n^{2}}
\end{aligned}
$$

die Koeffizienten einer absolut konvergenten Reihe (es wurde (1.6) und $\left|f_{\kappa}{ }^{(2 \lambda+2)}(t)\right| \leq K^{\prime}(\kappa, \lambda), 0 \leq t \leq 2 \lambda$, benutzt). Daher sind $c_{n}{ }^{k}$ die Gegenbauer-Stieltjes-Koeffizienten eines zonalen Maßes.

Dieses Ergebnis führt zu

Satz 5.3.3. Die Klassen $\mathscr{H}\left(\boldsymbol{X} ;-n^{2 \kappa}\right)\left(b z w . \mathscr{H}_{\boldsymbol{a}}\left(\boldsymbol{X} ;-n^{2 \kappa}\right)\right)$ und $\mathscr{H}\left(\boldsymbol{X} ;-[n(n+2 \lambda)]^{k}\right)\left(b z w . \mathscr{H}_{a}\left(\boldsymbol{X} ;-[n(n+2 \lambda)]^{k}\right)\right)(\kappa>0)$ sind gleich.

Beweis: Wir untersuchen zuerst den Fall $\boldsymbol{X}=\boldsymbol{L}^{1}\left(S^{k}\right)$ für die Klassen $\mathcal{H}$. Ist $f$ aus $\mathscr{H}\left(\boldsymbol{L}^{1} ;-n^{2 \lambda}\right)$, dann existiert nach der Definition ein $\mathrm{Maß} \mu_{1}$ aus $\boldsymbol{M}$, so daß gilt

$$
-n^{2 \kappa} Y_{n}(f ; x)=Y_{n}\left(d \mu_{1} ; x\right) \quad(n=0,1, \cdots) .
$$

Daraus erhält man für $n=1,2, \cdots$

$$
\begin{aligned}
-[n(n+2 \lambda)]^{\kappa} Y_{n}(f ; x) & =\left(\frac{n+2 \lambda}{n}\right)^{\kappa} Y_{n}\left(d_{\mu_{1}} ; x\right) \\
& =\frac{\lambda}{n+\lambda} c_{n}^{-\kappa} Y_{n}\left(d \mu_{1} ; x\right) .
\end{aligned}
$$

Nach Lemma 5.3.2 sind die $c_{n}^{-\kappa}$ die Koeffizienten eines zonalen Maßes und aus Lemma 5.3.1 folgt, daß ein $\mathrm{Maß} \mu_{2}$ aus $\boldsymbol{M}\left(S^{k}\right)$ existiert, so daß gilt

$$
\frac{\lambda}{n+\lambda} c_{n}^{-\kappa} Y_{n}\left(d \mu_{1} ; x\right)=Y_{n}\left(d \mu_{2} ; x\right) \quad(n=1,2, \cdots) .
$$

Mit $Y_{0}\left(d_{\mu_{2}} ; x\right)=0$ nach Voraussetzung folgt, daß $f$ in $\mathscr{H}\left(\boldsymbol{L}^{1} ;-[n(n+\right.$ $2 \lambda)]^{\kappa}$ ) enthalten ist.

Ist umgekehrt $f \in \mathscr{H}\left(\boldsymbol{L}^{1} ;-[n(n+2 \lambda)]^{\kappa}\right)$, dann erhält man aus 


$$
-[n(n+2 \lambda)]^{\kappa} Y_{n}(f ; x)=Y_{n}\left(d_{\mu} ; x\right) \quad(n=0,1, \cdots)
$$

die Beziehung

$$
-n^{2 \kappa} Y_{n}(f ; x)=\frac{\lambda}{n+\lambda} c_{n}{ }^{\kappa} Y_{n}(d \mu ; x)
$$

und damit ist $f$ aus $\mathscr{H}\left(\boldsymbol{L}^{1} ;-n^{2 \kappa}\right)$.

Der Beweis für die anderen Räume und Klassen folgt aus der Tatsache, daß die Folge $\left\{c_{n}{ }^{\kappa}\right\}$ in $(\boldsymbol{C}, \boldsymbol{C})$ und $\left(\boldsymbol{L}^{p}, \boldsymbol{L}^{p}\right)(1 \leq \boldsymbol{p} \leq \infty)$ enthalten ist (siehe Lemma 5.3.1 und (5.3.7)).

Wir kommen nun zu Anwendungen auf fraktionierte Integration. R. Askey und St. Wainger ([4, I, S. 218/219]) haben die sphärische fraktionierte Integration der Ordnung $\alpha, 0<\alpha<2 \lambda+1$, für die Funktionen $g$ aus $\boldsymbol{L}^{1}\left(S^{k}\right)$ definiert durch

$$
I_{\alpha}(g ; x)=c+\left(g * k_{a}\right)(x),
$$

wobei die zonale Funktion $k_{a}$ aus $\boldsymbol{L}_{\lambda}{ }^{1}$ durch die Entwicklung

$$
k_{\infty}(\cos \theta) \sim \sum_{n=1}^{\infty} \frac{n+\lambda}{\lambda}[n(n+2 \lambda)]^{-\infty / 2} P_{n}^{\lambda}(\cos \theta)
$$

definiert ist. Der Fall $\alpha=2$ wurde schon in Abschnitt 4.1, Lemma 4.1.5, untersucht; die Funktion $k_{2}(\cos \theta)$ stimmt mit $k(1, \cos \theta)$ in (4.1.27) überein.

$I_{\alpha}(g ; x)$ besitzt wegen der Darstellung für $k_{\alpha}(\cos \theta)$ die Entwicklung

$$
I_{a}(g ; x) \sim c+\sum_{n=1}^{\infty}[n(n+2 \lambda)]^{-\infty / 2} Y_{n}(g ; x) .
$$

(Diese Definition erfüllt im Gegensatz zu den von N. du Plessis [58] die Halbgruppen-Eigenschaft $I_{\alpha+\beta}(g ; x)=I_{\omega}\left(I_{\beta} g ; x\right)$ ).

Aus dieser Definition folgt z.B., daß alle Funktionen aus der Klasse $\mathscr{H}_{a}\left(X ;-[n(n+2 \lambda)]^{\kappa}\right)$ sich bis auf eine Konstante als fraktioniertes Integral der Ordnung $2 \kappa$ darstellen lassen. Für die Saturationsklasse $\mathscr{H}\left(X ;-[n(n+2 \lambda)]^{\kappa}\right)$ des verallgemeinerten Weierstraßintegrals $W_{t}^{*}$ gilt als Verallgemeinerung von Lemma 4.1.5

Lemma 5.3.4. Für ein $f \in \boldsymbol{X}$ gilt $f \in \mathcal{H}\left(\boldsymbol{X} ;-[n(n+2 \lambda)]^{\kappa}\right)$ genau dann, wenn 
für $f \in \boldsymbol{C}\left(S^{k}\right)$ gilt $f(x)=Y_{0}(f ; x)-\left(k_{\infty} * g\right)(x)$ mit $g \in \boldsymbol{L}^{\infty}\left(S^{k}\right)$, für $f \in \boldsymbol{L}^{p}\left(S^{k}\right), 1<p<\infty$, gilt $f(x)=Y_{0}(f ; x)-\left(k_{0} * g\right)(x)$ mit $g \in \boldsymbol{L}^{p}\left(S^{k}\right)$, fiur $f \in \boldsymbol{L}^{1}\left(S^{k}\right)$ gilt $f(x)=Y_{0}(f ; x)-\left(k_{\alpha} * d \mu\right)(x)$ mit $\mu \in \boldsymbol{M}$.

Der Beweis folgt sofort aus der Definition des Integrals $I_{\alpha}$.

Als Anwendung von Lemma 5.3.2 oder Satz 5.3.3 erhalten wir

Satz 5.3.5. Die Funktion $f$ aus $\boldsymbol{L}^{1}\left(S^{k}\right)$ ist darstellbar als fraktioniertes Integral der Ordnung $\alpha>0$ einer Funktion $g_{1} \in L^{1}\left(S^{k}\right)$ genau dann, wenn $f$ fast überall die folgende Darstellung mit einer Funktion $g_{2} \in \boldsymbol{L}^{1}\left(S^{k}\right)$ besitzt :

$$
\begin{aligned}
& f(x)=c+\left(g_{2} * h_{a}\right)(x), \\
& h_{\alpha}(\cos \theta) \sim \sum_{n=1}^{\infty} \frac{n+\lambda}{\lambda} n^{-\infty} P_{n}^{\lambda}(\cos \theta) .
\end{aligned}
$$

Die Funktion $h_{a}$ wurde ausführlich in $[49$, S. 81$]$ und in $[4, I]$ untersucht. $h_{a}$ besitzt die Darstellung

$$
h_{\alpha}(\cos \theta)=\frac{1}{\Gamma(\alpha)} \int_{0}^{1}\left[\frac{1-r^{2}}{\left(1-2 r \cos \theta+r^{2}\right)^{\lambda+1}}-1\right]\left(\log \frac{1}{r}\right)^{\alpha-1} \frac{d r}{r} \quad(\alpha>0) .
$$

$h_{a}(\cos \theta)$ ist stetig auf $0<\theta \leq \pi$ und außerdem ist $\left\|h_{\alpha}\right\|_{p, \lambda}<\infty$ für $1 \leq p<(2 \lambda+1) /(2 \lambda+1-\alpha)$. Für $\alpha>2 \lambda+1$ ist $h_{\infty}(\cos \theta)$ sogar stetig auf $[0, \pi]$ (s. [4, I, S. 214]). Für $\alpha=1$ ist $h_{1}(\cos \theta)=h(1, \cos \theta)$ nach Formel (4.3.12).

Folgerung 5.3.6. Alle Funktionen $f$ aus der Klasse $\mathcal{H}\left(\boldsymbol{X} ;-n^{2 \kappa}\right)$ bzw. $\mathscr{H}\left(\boldsymbol{X} ;-[n(n+2 \lambda)]^{\kappa}\right)$ sind darstellbar in der Form

$$
f(x)=Y_{0}(f ; x)-I_{2 \kappa}(g ; x)+c
$$

mit $g \in \boldsymbol{L}^{p}\left(S^{k}\right)$, falls $f \in \mathbb{L}^{p}\left(S^{k}\right), 1<p<\infty$, und $g \in \boldsymbol{L}^{\infty}\left(S^{k}\right)$, falls $f \in$ $C\left(S^{k}\right)$, oder

$$
f(x)=Y_{0}(f ; x)-I_{2 \kappa}(d \mu ; x),
$$

falls $f \in \boldsymbol{L}^{1}\left(S^{k}\right)$ mit $\mu \in \boldsymbol{M}\left(S^{k}\right)$ und $I_{2 \kappa}\left(d_{\mu} ; x\right)=\left(h_{2 \kappa} * d_{\mu}\right)(x)$ ist.

\section{4. Charakterisierungen für zonale Funktionen.}

Hier werden noch einmal die Klassen $\mathcal{H}_{a}(\boldsymbol{X} ;-n)$ und $\mathscr{H}_{a}(\boldsymbol{X}$; $-n(n+2 \lambda))$ untersucht, wenn wir zusätzlich voraussetzen, daß die 
Funktionen zonal sind. Die Ergebnisse entsprechen denen für periodische Funktionen. Die dort auftretende zweite Ableitung wird hier durch den Laplace-Beltrami-Operator für zonale Funktionen auf der Kugel ersetzt, die konjugierte Funktion durch ihre Verallgemeinerungen von B. Muckenhoupt und E. M. Stein. Wir beginnen mit dem letzten Fall und der Klasse $\mathscr{H}_{a}(\boldsymbol{X} ;-n)$. Wegen (1.12) und (5.3.1) ist dies die Klasse aller zonalen Funktionen $f \in X$, für die eine zonale Funktion $g \in X$ existiert, so $\operatorname{da} \beta-n \hat{f}(n)=\hat{g}(n), n=0,1,2, \cdots$, gilt.

Nach B. Muckenhoupt und E. M. Stein [49] führen wir zunächst die verallgemeinerten konjugierten Funktionen ein. Für Funktionen, die auf dem Einheitskreis in $E^{2}$ definiert und in eine Cosinusreihe entwickelbar sind, d.h.

$$
f(\theta) \sim \sum_{n=0}^{\infty} \hat{f}(n) \cos n \theta \quad(0 \leq \theta \leq \pi),
$$

ist das Poisson-Integral von $f$ gegeben durch (hier ist $x_{1}=r \cos \theta$, $\left.x_{2}=r \sin \theta\right)$

$$
u\left(x_{1}, x_{2}\right)=\sum_{n=0}^{\infty} \hat{f}(n) r^{n} \cos n \theta \quad(0 \leq r<1),
$$

welches im Inneren des Einheitskreises $x_{1}{ }^{2}+x_{2}{ }^{2}=1$ harmonisch ist und für $r \rightarrow 1$ - die Randwerte $f(\theta)$ annimmt. Die zu $u$ konjugierte harmonische Funktion $v\left(x_{1}, x_{2}\right)$ ist gegeben durch

$$
v\left(x_{1}, x_{2}\right)=\sum_{n=1}^{\infty} \hat{f}(n) r^{n} \sin n \theta \text {. }
$$

Der Grenzwert von $v\left(x_{1}, x_{2}\right)$ für $r \rightarrow 1$ - existiert fast überall und wird mit $\tilde{f}(\theta)$ bezeichnet.

Im $E^{k}$ bezeichnet man $k$ Funktionen $u_{1}, u_{2}, \cdots, u_{k}$ zueinander konjugiert, falls sie den verallgemeinerten Cauchy-Riemann-Differentialgleichungen genügen

$$
\sum_{i=1}^{k} \frac{\partial u_{i}}{\partial x_{i}}=0 ; \frac{\partial u_{i}}{\partial x_{j}}=\frac{\partial u_{j}}{\partial x_{i}} \quad\left(\left(x_{1}, x_{2}, \cdots, x_{k}\right) \in E^{k}\right) .
$$

Nimmt man speziell Funktionen $u_{1}$, die invariant bei Rotationen um die $x_{1}$-Achse sind, d.h. radial in den Variablen $\left(x_{2}, \cdots, x_{k}\right)$, und setzt man $y=\left(x_{2}{ }^{2}+x_{3}{ }^{2}+\cdots+x_{k}{ }^{2}\right)^{1 / 2}, x_{1}=x, u_{1}\left(x_{1}, \cdots, x_{k}\right)=u(x, y)$ und $u_{l}\left(x_{1}, \cdots, x_{k}\right)$ $=-x_{l} y^{-(2 \lambda+1)} v(x, y)(l=2, \cdots, k ; 2 \lambda=k-2)$ (diese spezielle Wahl ist 
möglich), dann geht (5.4.1) über in

$$
v_{x}=-y^{2 \lambda} u_{y} ; \quad v_{x}=y^{2 \lambda} u_{x} .
$$

In Polarkoordinaten $x=r \cos \theta$ und $y=r \sin \theta$ erhält man

$$
r v_{r}=-(r \sin \theta)^{2 \lambda} u_{\theta} ; \quad v_{\theta}=r(r \sin \theta)^{2 \lambda} u_{r} .
$$

Besitzt nun $f(\theta)$ die Entwicklung nach Gegenbauer-Polynomen

$$
f(\theta) \sim \sum_{n=0}^{\infty} \hat{f}(n) P_{n}^{\lambda}(\cos \theta)
$$

mit dem Poisson-Integral

$$
V_{r}(f ; \theta)=\sum_{n=0}^{\infty} \hat{f}(n) r^{n} P_{n}^{\lambda}(\cos \theta) \quad(0 \leq r<1,0 \leq \theta \leq \pi),
$$

dann ist das konjugierte Poisson-Integral $\tilde{V}_{r}$ definiert durch

$$
\widetilde{V}_{r}(f ; \theta)=2 \lambda \sum_{n=1}^{\infty}(n+2 \lambda)^{-1} \hat{f}(n) r^{n} \sin \theta P_{n-1}^{\lambda+1}(\cos \theta)
$$

$(0 \leq \theta \leq \pi)$.

Setzt man $u=V_{r}(f ; \theta), v=y^{2 \lambda} \widetilde{V}_{r}(f ; \theta)$, dann erfüllen $u$ und $v$ die Differentialgleichungen (5.4.2). Weiter gilt

Satz 5.4.1. Ist $f \in \mathbb{L}_{\lambda}{ }^{p}, 1<p<\infty$, dann gilt:

a)

$$
\left\|\widetilde{V}_{r} f\right\|_{p, \lambda} \leq A_{p}\|f\|_{p, \lambda} .
$$

b) Es existiert eine Funktion $\hat{f}(\theta) \in \mathbb{L}_{\lambda}{ }^{p}$ mit

$$
\lim _{r \rightarrow 1-}\left\|\widetilde{V}_{r} f-\tilde{f}\right\|_{p, \lambda}=0
$$

und

$$
\|\tilde{f}\|_{p, \lambda} \leq A_{p}\|f\|_{p, \lambda}
$$

c)

$$
\lim _{r \rightarrow 1-} \tilde{V}_{r}(f ; \theta)=\tilde{f}(\theta)
$$

für fast alle $\theta$.

Dies ist eine Verallgemeinerung eines Satzes von M. Riesz. Mit Hilfe dieses wichtigen Satzes 5.4.1 beweisen wir nun

Satz 5.4.2. Sind die Funktionen $f$ und $g$ aus $\mathbb{L}_{\lambda}^{p}, 1 \leq p<\infty$, bzw. $C_{\lambda}$, dann gilt

$$
-n \hat{f}(n)=\hat{g}(n)
$$$$
(n=0,1, \cdots)
$$

genau dann, wenn $\tilde{f}$ aus $\boldsymbol{L}_{\lambda}{ }^{p}$ ist, $(\sin \theta)^{2 \lambda} \tilde{f}(\theta)$ absolut stetig auf $[0, \pi]$ ist, in den Punkten 0 und $\pi$ verschwindet, und wenn gilt 


$$
\frac{d}{d \theta}\left[(\sin \theta)^{2 \lambda} \tilde{f}(\theta)\right]=-(\sin \theta)^{2 \lambda} g(\theta) \quad\left(g \in \boldsymbol{L}_{\lambda}^{p}\right)
$$

Beweis: Wir nehmen an, daß für zwei Funktionen $f$ und $g$ aus $\boldsymbol{L}_{\lambda}{ }^{p}(5.4 .5)$ erfüllt ist. Wie oben erwähnt, genügen die Funktionen $u=V_{r}(f ; \theta)$ und $v=(r \sin \theta)^{2 \lambda} \widetilde{V}_{r}(f ; \theta)$ den Gleichungen (5.4.2). Aus der zweiten erhält man

$$
\begin{gathered}
\frac{d}{d \theta}\left[(r \sin \theta)^{2 \lambda} \tilde{V}_{r}(f ; \theta)\right]=r(r \sin \theta)^{2 \lambda} \frac{\partial}{\partial r} V_{r}(f ; \theta) \\
=-(r \sin \theta)^{2 \lambda} V_{r}(g ; \theta)
\end{gathered}
$$

wegen (5.4.3) und (5.4.5). Wir behandeln nun den Fall $\boldsymbol{L}_{\lambda}{ }^{1}$ (Sind $f$ und $g$ aus $\boldsymbol{L}_{\lambda}^{p}, p>1$, dann sind sie ebenfalls aus $\left.\boldsymbol{L}_{\lambda}{ }^{1}\right)$. Aus (5.4.5) folgt, daß $f$ die Darstellung

$$
f(\theta)=\hat{f}(0)-I_{1}(g ; \cos \theta)+c
$$

besitzt. Dies ist die Aussage der Folgerung 5.3.6 oder Lemma 4.3.2. Nach Satz 5.3.5 ist $I_{1}(g ; \cos \theta)=\left(g_{2} * h_{1}\right)(\cos \theta)$ mit einer Funktion $g_{2} \in \boldsymbol{L}_{\lambda}{ }^{1}$ und $h_{1} \in \boldsymbol{L}_{\lambda}{ }^{p}$ mit $1<p(\lambda)<1+(1 / 2 \lambda)$, woraus $\left\|I_{1} g\right\|_{p, \lambda} \leq\left\|g_{2}\right\|_{1, \lambda}$ $\left\|h_{1}\right\|_{p, \lambda}$, also $f \in \mathcal{L}_{\lambda}{ }^{p}$ für ein $p(\lambda)>1$ folgt. Nach Satz 5.4.1 ist auch $\tilde{f} \in \boldsymbol{L}_{\lambda}{ }^{p}, p(\lambda)>1$, also $\tilde{f} \in \boldsymbol{L}_{\lambda}{ }^{1}$. Integration von (5.4.7) liefert

$$
(\sin \theta)^{2 \lambda} \widetilde{V}_{r}(f ; \theta)=-\int_{0}^{\theta}(\sin \tau)^{2 \lambda} V_{r}(g ; \tau) d \tau .
$$

Für $r \rightarrow 1-$ konvergiert die linke Seite nach Satz 5.4.1 c) fast überall gegen $(\sin \theta)^{2 \lambda} \tilde{f}(\theta)$. Wegen

$$
\begin{aligned}
& \left|\int_{0}^{\theta}(\sin \tau)^{2 \lambda}\left[V_{r}(g ; \tau)-g(\tau)\right] d \tau\right| \\
& \quad \leq\left\|V_{r} g-g\right\|_{1, \lambda}=o(1)
\end{aligned}
$$

konvergiert die rechte Seite der Gleichung überall gegen $-\int_{0}^{\theta}(\sin \tau)^{2 \lambda} g(\tau) d \tau$, also gilt fast überall

$$
(\sin \theta)^{2 \lambda} \tilde{f}(\theta)=-\int_{0}^{\theta}(\sin \tau)^{2 \lambda} g(\tau) d \tau .
$$

Für $\theta=0$ ist die rechte Seite Null und für $\theta=\pi$ gilt dies ebenfalls, da $\hat{g}(0)=0$ ist. Außerdem folgt fast uiberall 


$$
\frac{d}{d \theta}\left[(\sin \theta)^{2 \lambda} \widetilde{f}(\theta)\right]=-(\sin \theta)^{2 \lambda} g(\theta)
$$

und die eine Richtung von Satz 5.4.2 ist für $\boldsymbol{L}_{\wedge}{ }^{1}$ bewissen. Sind $f$ und $g$ aus $\boldsymbol{L}_{\lambda}{ }^{p}, p>1$ bzw. aus $\boldsymbol{C}_{\lambda}$, dann folgt diese Richtung des Beweises entsprechend.

Es ist also noch die Umkehrung zu beweisen. Aus der Beziehung (5.4.6) erhält man

$$
\begin{aligned}
\hat{g}(n) & =c(n, \lambda) \int_{0}^{\pi} g(\theta) P_{n}^{\lambda}(\cos \theta)(\sin \theta)^{2 \lambda} d \theta \\
& =-c(n, \lambda) \int_{0}^{\pi} P_{n}^{\lambda}(\cos \theta) \frac{d}{d \theta}\left[(\sin \theta)^{2 \lambda} \tilde{f}(\theta)\right] d \theta .
\end{aligned}
$$

Partielle Integration der rechten Seite führt $\mathrm{zu}$

$$
\hat{g}(n)=-2 \lambda c(n, \lambda) \int_{0}^{\pi}(\sin \theta)^{2 \lambda+1} \tilde{f}(\theta) P_{n-1}^{\lambda+1}(\cos \theta) d \theta,
$$

wenn man berücksichtigt, $\operatorname{da} \beta(\sin \theta)^{2 \lambda} \tilde{f}(\theta)$ für $\theta=0$ und $\theta=\pi$ verschwindet und (4.2.18). Nun ist $(\sin \theta)^{2 \lambda} \tilde{f}(\theta)$ absolut stetig und besitzt die Darstellung (5.4.9).

Daraus folgt

$$
|\widetilde{f}(\theta)| \leq\|g\|_{1, \lambda}(\sin \theta)^{-2 \lambda}
$$

und daher $\tilde{f} \in \boldsymbol{L}_{\lambda}{ }^{p}$ für ein $p(\lambda)$ mit $1<p(\lambda)<1+(1 / 2 \lambda)$. Die Funktion $\tilde{f}$ ist also hier in $\boldsymbol{L}_{\lambda}{ }^{p}$ für ein $p>1$, wenn $\tilde{f}$ nur aus $\boldsymbol{L}_{\lambda}{ }^{1}$ vorausgesetzt ist. Damit gilt

$$
\begin{aligned}
& \int_{0}^{\pi}\left[\tilde{f}(\theta)-\tilde{V}_{r}(f ; \theta)\right] P_{n-1}^{\lambda+1}(\cos \theta)(\sin \theta)^{2 \lambda+1} d \theta \\
& \leq K(n) \cdot\left\|\tilde{V}_{r} f-\tilde{f}\right\|_{p, \lambda}=o(1)
\end{aligned}
$$

für $r \rightarrow 1-$, wobei $p=p(\lambda)>1$ ist. Wir untersuchen nun

$$
-2 \lambda c(n, \lambda) \int_{0}^{\pi} \widetilde{V}_{r}(f ; \theta) P_{n-1}^{\lambda+1}(\cos \theta)(\sin \theta)^{2 \lambda+1} d \theta
$$

Setzt man für $\widetilde{V}_{r}(f ; \theta)$ die Reihe (5.4.4) ein, integriert gliedweise, berücksichtigt die Orthogonalität der Funktionen $P_{n-1}^{\lambda+1}(\cos \theta)$ bezüglich der Gewichtsfunktion $(\sin \theta)^{2(\lambda+1)}$ und die Beziehung $([32, \mathrm{I}, \mathrm{S}$. 177]) 


$$
\int_{0}^{\pi}\left[P_{n-1}^{\lambda+1}(\cos \theta)\right]^{2}(\sin \theta)^{2(\lambda+1)} d \theta=[c(n-1, \lambda+1)]^{-1},
$$

dann ist (5.4.12) gleich

$$
-c(n, \lambda) \cdot \frac{(2 \lambda)^{2}}{n+2 \lambda} \hat{f}(n) r^{n} \cdot[c(n-1, \lambda+1)]^{-1}=-n \tilde{f}(n) r^{n}
$$

Wegen (5.4.11) konvergiert dies gegen die rechte Seite von (5.4.10), also ist $-n \hat{f}(n)=\hat{g}(n)$, womit der Satz bewiesen ist.

Dieser Satz ist ein neues Ergebnis. Nur im Falle $\lambda=1 / 2$ (Legendre-Polynome) und $\boldsymbol{L}_{1 / 2}^{2}$ ist eine ähnliche Aussage ohne Verwendung konjugierter Funktionen bekannt (s. [37, S. 611]). Führt man in den Ergebnissen von Satz 5.4.2 formal den Grenzwert $\lambda \rightarrow 0+$ durch, dann werden die $\hat{f}(n)$ und $\hat{g}(n)$ wegen (1.5) die Koeffizienten einer Cosinusreihe und (5.4.6) geht in das bekannte Ergebnis $\frac{d}{d \theta} \tilde{f}(\theta)=-g(\theta)$ über (vgl. z.B. [19, Sec. 1.5]).

Als letzten Fall untersuchen wir die Klasse $\mathcal{H}_{a}(X ;-n(n+2 \lambda))$.

Satz 5.4.3. Sind die Funktionen $f$ und $g$ aus $\mathbb{L}_{\lambda}^{p}, 1 \leq p<\infty$ oder $\mathbb{C}_{\lambda}$, dann gilt

$$
-n(n+2 \lambda) \hat{f}(n)=\hat{g}(n) \quad(n=0,1,2, \cdots)
$$

genau dann, wenn $f(\theta)$ lokal absolut stetig auf $(0, \pi)$ ist, die Funktion $(\sin \theta)^{2 \lambda}(d / d \theta) f(\theta)$ absolut stetig auf $[0, \pi]$ ist und in den Punkten 0 und $\pi$ verschwindet, und wenn gilt

$$
\frac{d}{d \theta}\left[(\sin \theta)^{2 \lambda} \frac{d}{d \theta} f(\theta)\right]=(\sin \theta)^{2 \lambda} g(\theta) \text {. }
$$

Beweis: Die Differentialgleichung (1.2) geht für die zonalen Kugelfunktionen $P_{n}^{\lambda}(\cos \theta)$ über in

$$
\begin{gathered}
\Delta_{\lambda} P_{n}^{\lambda}(\sin \theta) \equiv(\sin \theta)^{-2 \lambda} \frac{d}{d \theta}\left[(\sin \theta)^{2 \lambda} \frac{d}{d \theta} P_{n}^{\lambda}(\cos \theta)\right] \\
=-n(n+2 \lambda) P_{n}^{\lambda}(\cos \theta) .
\end{gathered}
$$

Setzt man (5.4.13) voraus, dann folgt für das Weierstraßintegral (4.1.6) $W_{t}(f ; \theta)$ für zonale Funktionen $\Delta_{\lambda} W_{t}(f ; \theta)=W_{t}(g ; \theta)$. Zweimaglige Integration führt $\mathrm{zu}$ 


$$
W_{t}(f ; \theta)=W_{t}(f ; \varepsilon)+\int_{\varepsilon}^{\theta}(\sin \eta)^{-2 \lambda} d \eta \int_{0}^{\eta}(\sin \tau)^{2 \lambda} W_{t}(g ; \tau) d \tau .
$$

Aus der Normkonvergenz von $W_{t} f$ gegen $f$ für $t \rightarrow 0+$ folgt die Existenz einer Teilfolge $t_{i}$, so daß $W_{t_{i}}(f ; \theta)$ fast überall gegen $f(\theta)$ für $t_{i} \rightarrow 0+$ konvergiert. Daraus folgt

$$
f(\theta)=f(\varepsilon)+\int_{\varepsilon}^{\theta}(\sin \eta)^{-2 \lambda} d \eta \int_{0}^{\eta}(\sin \tau)^{2 \lambda} g(\tau) d \tau,
$$

wobei die rechte Seite für jedes feste $\varepsilon>0$ und für alle $\theta \in(0, \pi)$ konvergiert. Also ist $f(\theta)$ differenzierbar auf $(0, \pi)$ und

$$
\frac{d}{d \theta} f(\theta)=(\sin \theta)^{-2 \lambda} \int_{0}^{\theta}(\sin \tau)^{2 \lambda} g(\tau) d \tau .
$$

Die Funktion $(\sin \theta)^{2 \lambda}(d / d \theta) f(\theta)$ ist daher absolut stetig auf $[0, \pi]$, verschwindet für $\theta=0$ und nach der Voraussetzung $\hat{g}(0)=0$ für $\theta=\pi$, und es gilt

$$
\Delta_{\lambda} f(\theta)=g(\theta)
$$

fast überall (die Aussagen gelten überall, wenn $f$ aus $\boldsymbol{C}_{\lambda}$ ist) d.h. (5.4.14) ist erfüllt.

Zum Beweis der Umkehrung geht man von (5.4.14) aus, woraus

$$
\hat{g}(n)=c(n, \lambda) \int_{0}^{\pi} P_{n}^{\lambda}(\cos \theta) \frac{d}{d \theta}\left[(\sin \theta)^{2 \lambda} \frac{d}{d \theta} f(\theta)\right] d \theta
$$

folgt. Zweimalige partielle Integration (oder formal die zweite Greensche Identität für zonale Funktionen [65, S. 213]) führt zu

$$
\begin{aligned}
\hat{g}(n) & =c(n, \lambda) \int_{0}^{\pi} \frac{d}{d \theta}\left[(\sin \theta)^{2 \lambda} \frac{d}{d \theta} P_{n}^{\lambda}(\cos \theta)\right] f(\theta) d \theta \\
& =-c(n, \lambda) \int_{0}^{\pi} n(n+2 \lambda) P_{n}^{\lambda}(\cos \theta) f(\theta)(\sin \theta)^{2 \lambda} d \theta \\
& =-n(n+2 \lambda) \hat{f}(n) .
\end{aligned}
$$

Die integrierten Anteile verschwinden jeweils für $\theta=0$ und $\theta=\pi$. Damit ist der Satz bewiesen.

Für $\lambda=0$ gehen die Aussagen in das bekannte Ergebnis von P. L. Butzer [15], P. L. Butzer und G. Sunouchi [25] über. 


\section{Literaturverzeichnis}

[1] Alexits, G., Sur l'ordre de grandeur de l'approximation d'une fonction par les moyennes de sa série de Fourier, Mat. Fiz. Lapok 48 (1941), 410-433.

[2] - Sur l'ordre de grandeur de l'approximation d'une fonction périodique par les sommes de Fejér, Acta Math. Acad. Sci. Hung. 3 (1952), 29-40.

[3] Askey, R., Norm inequalities for some orthogonal series, Bull. Amer. Math. Soc. 72 (1966), 808-823.

[4] Askey, R. and St. Wainger, On the behaviour of special classes of ultraspherical expansions, I, II, J. Analyse Math. 15 (1965), I, : 93-220 ; II, 221-244.

[5] Berens, H., Approximationssätze für Halbgruppenoperatoren in intermediären Räumen, Schr. Math. Inst. Univ. Münster 32 (1964), 1-60.

[6] Berens, H. und E. Görlich, Über einen Darstallungssatz für Funktionen als Fourierintegrale und Anwendungen in der Fourieranalysis, Tôhoku Math. J. 18 (1966), 429-453.

[7] Bochner, S., Quasi-analytic functions, Laplace operator, positive kernels, Ann. of Math. 51 (1950), 68-91.

[8] - Positive zonal functions on spheres, Proc. Nat. Acad. Sci. U.S.A. 40 (1954), 1141-1147.

[9] - Sturm-Liouville and heat equations whose eigenfunctions are ultraspherical polynomials or associated Bessel functions, Proceedings of the Conference on Differential Equations, 23-48, University of Maryland, 1955.

[10] Bohman, H., On approximation of continnous and of analytic functions, Ark. Mat. 2 (1952), 43-56.

[11] Buchwalter, H., Saturation de certains procédés de sommation, C.R. Acad. Sci. Paris 248 (1959), 909-912.

[12] - Saturation dans un espace normé, C. R. Acad. Sci. Paris 250 (1960), 651-653.

[13] Butzer, P.L., Über den Grad der Approximation des Identitätsoperators durch Halbgruppen von linearen Operatoren und Anwendungen auf die Theorie der singulären Integrale, Math. Ann. 133 (1957), 410-425.

[14] -, Fourier transform methods in the theory of approximation, Arch. Rat. Mech. Anal. 5 (1960), 390-415.

[15] - Beziehungen zwischen den Riemannschen, Taylorschen und gewöhnlichen Ableitungen reellwertiger Funktionen, Math. Ann. 144 (1961), 275-298.

[16] - Das Randverhalten harmonischer Funktionen und die endliche Legendre-Transformation, Unveröffentl. Manuskript, 1961.

[1.] - The dependence of the solution of the equation of heat conduction upon its initial temperature distribution, asymptotic expansions, Arch. Math. (Basel) 13 (1962), 302-312.

[18] - Integral transform methods in the the theory of approximation: Über Approximationstheorie, Basel, 1964, 12-23.

[19] Butzer, P. L. and H. Berens, Semi-groups of Operators and Approximation, Grundlehren der Math. Wiss. 145, Springer, Berlin, 1967.

[20] Butzer, P. L. und E. Göriich, Saturationsklassen und asymptotische Eigenschaften trigonometrischer singulärer Integrale, Festschrift zur Gedenkfeier für Karl Weierstraß, 339-392, Westdeutscher Verlag, KöIn, 1965.

[21] Butzer, P. L. und C. Müller, Über die Approximation vermittels Kugelfunktionen beliebiger Dimension, Unveröffentl. Manuskript, 1962.

[22] Butzer, P. L. and R. J. Nessel, Favard classes for $n$-dimensional singular integrals, Bull. Amer. Math. Soc. 72 (1966), 493-498. 
[23] Butzer, P. L. and R. J. Nessel, Contributions to the theory of saturation for singular integrals in several variables, I, Nederl. Akad. Wetensch. Proc. Ser. A 69 (1966), 515-531.

[24] Butzer, P. L. und S. Pawelke, Ableitungen von trigonometrischen Approximationsprozessen, Acta Sci. Math, 28 (1967), 173-183.

[25] Butzer, P. L. and G. Sunouchi, Approximation theorems for the solution of Fourier's problem and Dirichlet's problem, Math. Ann. 155 (1964), 316-330.

[26] Calderón, A. P., Intégrales singulares y sus aplicaciones a ecuaciones diferenciales hiperbolicas, Universidad de Buenos Aires, 1960.

[27] Calderón, A. P. and A. Zygmund, On a problem of Mihlin, Trans. Amer. Math. Soc. 78 (1955), 209-224.

[28] Chen, K. K., On the Cesàro-summability of the Laplace's series of hyperspherical functions, Sci. Rep. Tôhoku Imp. Univ. Ser. 1, 17 (1928), 1073-1089.

[29] Dunford, N. and J. T. Schwartz, Linear Operators, I, New York, 1958.

[30] Dunkl, C. F., Operators and harmonic analysis on the sphere, Trans. Amer. Math. Soc. 125 (1966), 250-263.

[31] Džafarov, A. S., Über die Ordnung der besten Approximation stetiger Funktionen auf der Einheitskugel durch endliche sphärische Summen (russisch), Studies Contemporary Problems Constructive Theory of Functions (Proc. Second AllUnion Conf., Baku 1962), 46-52, Baku, 1965.

[32] Erdélyi, A., Higher Transcendental Functions, I, II, New York, 1953.

[33] Favard, J., Sur I'approximation des fonctions d'une variable réelle, Colloque d'Anal. Harmon. Publ. C. N. R. S. Paris. 15 (1949), 97-110.

[34] - Sur la saturation des procédés de sommation, J. Math. Pures Appl. 36 (1957), 359-372.

[35] Görlich, E., Distributionentheoretische Methoden in der Saturationstneoris, Dissertation, Aachen, 1967.

[36] Görlich, E., und R. J. Nessel, Über Peano-und Riemann-Ableitungen in der Norm, Arch. Math. (Basel) 18 (1967), 399-410.

[37] Hille, E. and R. S. Phillips, Functional Analysis and Semi-groups, Amer. Math. Soc. Coll. Publ. 31, New York, 1957.

[38] Hirschman, I. I. Jr., Harmonic analysis and ultraspherical polynomials, Proceedings of the Conference on Harmonic Analysis, Cornell, 1956.

[39] Kaczmarz, St. und H. Steinhaus, Theorie der Orthogonalreihen, Warschau, 1935.

[40] Kogbetliantz, E., Recherches sur la sommabillité des séries ultrasphériques par la méthode des moyennes arithmétiques, J. Math. Pures Appl. (3) 9 (1924), 107-187.

[41] Korovkin, P. P., Linear Operators and Approximation Theory, Delhi, 1960.

[42] Koschmieder, L., Über die $C$-Summierbarkeit gewisser Reihen von Didon und Appell. Math. Ann. 104 (1931). 387-402.

[43] Kušnirenko, G. G. Über die Approximation von auf der Einheitskugel definierten Funktionen durch endliche sphärische Summen (russisch), Naučn. Dokl. Vysš. Školy Fiz.-Mat. Nauki No. 4 (1958), 47-53.

[44] Kunirenko, G. G., Einige Fragen der Approximation stetiger Funktionen auf der Einheitskugel durch endliche sphärische Summen, Trudy Charkov. Politechn. Inst. Ser. Inž. Fiz. 25 No. 3 (1959), 3-22.

[45] Kuttner, B., On positive Riesz and Abel typical means, Proc. London Math. Soc. Ser. 2, 49 (1947), 328-352.

[46] de Leeuw, K., On the adjoint semi-group and some problems in the theory of approximation, Math. Z. 73 (1960), 219-234.

[47] Lorentz, G. G. Approximation of Functions, New York, 1966.

[48] McShane, E. J. and T. A. Botts, Real Analysis, Princeton, 1959 
[49] Muckenhoupt, B. and E. M. Stein, Classical expansions and their relations to conjugate harmonic functions, Trans. Amer. Math. Soc. 118 (1965). 17-92.

[50] Müller, C., Über die ganzen Lösungen der Wellengleichung (Nach einem Vortrag von Herglotz), Math. Ann. 124 (1952), 235-264.

[51] — Spherical harmonics, Lecture Notes in Mathematics 17, Berlin, 1966.

[52] Nessel, R. J., Das Saturationsproblem für mehrdimensionale singuläre Integrale und seine Lösung mit Hilfe der Fourier Transformation, Dissertation, Aachen, 1965.

[53] - Contributions to the theory of saturation for singular integrals in several variables, II, III, Nederl. Akad. Wetensch. Proc. Ser. A 70 (1967), II, 52-64; III, 65-73.

[54] Nessel, R. J. und A. Pawelke, Über Favardklassen von Summationsprozessen mehrdimensionaler Fourierreihen, Compositio Math. 19 (1968), 196-212.

[55] Newman, D. J. and H. S. Shapiro, Jackson's theorem in higher dimensions : Über Approzimationstheorie, Basel, 1964, 208-219.

[56] Newman, J. and W. Rudin, Mean convergence of orthogonal series, Proc. Amer. Math. Soc. 3 (1952), 219-222.

[57] Niemeyer, H., Lokale und asymptotische Eigenschaften der Lösungen der Helmholtzschen Schwingungsgleichung, Jber. DMV 65 (1962), 1-44.

[58] du Plessis, N., Spherical fractional integrals, Trans. Amer. Math. Soc. 84 (1957), 262-272.

[59] Pollard, H., The mean convergence of orthogonal series, II, Trans, Amer. Math. Soc. 63 (1948), 355-367.

[60] Ragozin, D. L., Approximation theory on compact manifolds and Lie groups, with applications to harmonic analysis, unpubl. Ph. D. Thesis, Harvard Univ., Cambridge, Mass., 1967.

[61] Rudin, W., Uniqueness theory for Laplace series, Trans. Amer. Math. Soc. 68 (1950), 287-303.

[62] Sansone, G., Orthogonal Functions, New York, 1959.

[63] Seeley, R. T., Spherical Harmonics, Amer. Math. Monthly 73 (1966), 115-121.

[64] Shapiro, V. L., The symmetric derivative on the $(k-1)$-dimensional hypersphere, Trans. Amer. Math. Soc. 81 (1956), 514-524.

[65] , Localization on spheres, Trans. Amer. Math. Soc. 86 (1957), 212-219.

[66] Soc., No. 39, 1961.

[67] Siegel, A., Summability $C$ of series of surface spherical harmonics, Trans, Amer. Math. Soc. 104 (1962), 284-307.

[68] Sunouchi, G., On the class of saturation in the theory of approximation, II, Tôhoku Math. J. 13 (1961), 112-118.

[69] Characterization of certain classes of functions, Tôhoku Math. J. 14 (1962), 127-134.

[70] Sunouchi, G. and C. Watari, On determination of the class of saturation in the theory of approximation, I, Proc. Japan Acad. 34 (1958), 477-481.

[71] Taibleson, M. H., On the theory of Lipschitz spaces of distributions on Euclidean $n$-space, J. Math. Mech. 13 (1964), 407-479.

[72] Zamansky, M., Classes de saturation de certains procédés d'approximation des séries de Fourier des fonction continues, Ann. Sci. École Norm. Sup. 66 (1949), 19-93.

[73] , Sur la sommation des séries de Fourier derivées, C. R. Acad. Sci. Paris 231 (1950), 1118-1120.

[74] Zygmund, A., Trigonometric Series I, Cambridge, 1959. 